Draft version 2.0, -Dec-2020. This paper has not been peer reviewed.

Please do not copy or cite without author's permission

This submission contains (1) main article and (2) supplementary material.

\title{
1 Characterizing the time course of decision-making in change
}

\author{
Anthea G. Blunden, Dylan Hammond, Piers D. L. Howe, and Daniel \\ R. Little \\ The University of Melbourne
}

Abstract

We propose a novel modeling framework for characterizing the time course of change detection based on information held in visual short-term memory. Specifically, we seek to answer whether change detection is better captured by a first-order integration model, in which information is pooled from each location, or a second-order integration model, in which each location is processed independently. We diagnose whether change detection across locations proceeds in serial or parallel and how processing is affected by the stopping rule (i.e., detecting any change versus detecting all changes; Experiment 1) and how the efficiency of detection is affected by the number of changes in the display (Experiment 2). We find that although capacity is generally limited in both tasks, architecture varies from parallel self-terminating in the OR task to serial self-terminating in the AND task. Our novel framework allows model comparisons across a large set of models ruling out several competing explanations of change detection.

Keywords: Visual Short Term Memory, Change Detection, Decision-Making, Mental Architecture, Systems Factorial Technology

In the $15+$ years since Wilken and Ma (2004) introduced the continuous report paradigm, the major foci in the study of change detection have been (a) the development of models that differentiate "slot-based" accounts from the use of continuous resources (see e.g., Bays \& Husain, 2008; Wilken \& Ma, 2004), (b) the development of mixture models which encompass elements of guessing and information-based responding (Cowan \& Rouder, 2009; Zhang \& Luck, 2008), and (c) the characterization of capacity limits in the visual short-term memory processes that underlie change detection (Alvarez \& Cavanagh, 2004; Cowan, 2001; Luck \& Vogel, 1997; Pashler, 1988; Phillips, 1974). Although this body of work has provided insight into the structure and formation of memory representations, the other primary question posed in Wilken and Ma's paper "how is information from different possible change locations integrated?" has been largely ignored. However, the answer to the question of information integration would provide fundamental insight into how change detection decisions occur. Namely, is change detection better explained by a first-order, differencing model (Sorkin, 1962), in which the evidence from each location is summed or 
pooled into a single source of evidence or by a second-order, independent decisions model, in which each location is considered in parallel (Noreen, 1981; Shaw, 1982)?

In more detail, as in related tasks like detection or visual search, first-order integration models assume that each location or feature value is represented by a noisy strength variable of differing magnitude. Higher magnitudes correspond to stronger evidence for the presence of a stimulus. Evidence from each location is pooled together and compared to a single decision criterion. If the pooled evidence exceeds some threshold value, a target present decision is made. Second-order integration models assume that information from each location is processed separately and independently. The strength of each location is compared to its own criterion. A target present response is made when either criterion is exceeded. Gardner's (1973) independent channels model and Smith's (1998) separable decisions model are both notable examples of second-order models. If the same criterion is used at all locations, the model is equivalent to assuming that only the location or feature with the largest strength is evaluated (Koopman, 1956; Smith, 2010). That is, the probability that any of the locations exceeds the criterion is the same as the probability that the location with maximum strength exceeds the criterion. Graham, Kramer, and Yager's (1987) maximum of outputs model or MAX model is one such model which assumes that the location with maximum output is selected via pre-attentive filtering and then compared to a decision criterion. The MAX model provides a good description of performance in cued detection and visual search tasks (see Smith \& Sewell, 2013, for a review). In the present paper, we seek to understand how these models apply to the detection of changes in different locations.

Both first- and second-order models can be instantiated as signal detection models and then compared using, for instance, receiver operating characteristics (ROCs; Wilken \& Ma, 2004). A limitation of this approach is that the models only predict or account for choice probabilities or accuracy, without taking into account response time. Further, the

\title{
Address correspondence to:
}

\author{
Anthea G. Blunden (anthea.blunden@unimelb.edu.au) or \\ Daniel R. Little (daniel.little@unimelb.edu.au) \\ The Melbourne School of Psychological Sciences \\ The University of Melbourne \\ Parkville, VIC \\ AUSTRALIA 3010
}

\section{Acknowledgements}

We would like to thank Danièle Martinie for assistance in data collection and Dr Robert De Lisle for assistance with programming. Portions of this paper were completed as part of a PhD thesis by Dr Anthea Blunden and as part of an Honours Thesis by Dylan Hammond. This work was supported by ARC grant DP160102360 awarded to A/Prof Daniel R. Little and by an Australian Government Research Training Program Scholarship awarded to Anthea Blunden. We thank Chris Donkin and two anonymous reviewers for comments on an earlier draft of this paper. We also thank Philip Smith and Simon Lilburn for helpful discussions on this work, and Peter Shepherdson for many helpful suggestions including the idea for the MAX-first model. 
predictions of the first- and second-order models make qualitatively similar ROC predictions and are therefore difficult to distinguish on the basis of detection accuracy alone. Here, we develop a framework for generalizing first- and second-order models of change detection across two locations to account for response time.

To foreshadow our approach and the major contribution of our present work, we present a set of novel parametric models that combine various decision architectures (e.g., serial, parallel, or pooled coactivation) with different decision stopping rules. We also introduce a novel methodology which makes use of the strong inference techniques available via Systems Factorial Technology (SFT; Little, Altieri, Fifić, \& Yang, 2017; Townsend \& Nozawa, 1995; Townsend \& Wenger, 2004) to provide converging non-parametric analyses to distinguish the models. In generalizing first- and second-order models to response time, it is necessary to consider (a) how the representation of the strength of a change develops at each potential location, (b) how that information is integrated, and (c) how the resulting evidence is accumulated from that representation over time. We draw on a number of recent developments in perceptual categorization (Blunden, Wang, Griffiths, \& Little, 2015; Fifić, Little, \& Nosofsky, 2010; Fifić, Nosofsky, \& Townsend, 2008; Little, Nosofsky, \& Denton, 2011; Little, Nosofsky, Donkin, \& Denton, 2013; Moneer, Wang, \& Little, 2016; see Griffiths, Blunden, \& Little, 2017 for a review) and models that have synthesized multidimensional signal detection (e.g., Generalized Recognition Theory, GRT; Ashby \& Townsend, 1986; Townsend, Houpt, \& Silbert, 2012) with decision-bound theories (Ashby \& Gott, 1988), sequential sampling models (Busemeyer, 1985; Link, 1992; Luce, 1986; Ratcliff, 1978; Ratcliff \& Rouder, 1998; Townsend \& Ashby, 1983), and mental architectures (Kantowitz, 1974; Schweickert, 1992; Schweickert, Fisher, \& Sung, 2012; Sternberg, 1969; Townsend, 1984).

At each location, the strength of the change or "change signal" is computed by taking the difference between Gaussian distributions, one representing the memory array and the other representing the probe array. In a first-order model, the pooled strength is represented as a joint distribution over all possible locations. In a second-order model, the change at each location is represented independently. From these distributions we derive a change signal which is then used as the sampling or drift rate in an evidence accumulation process. Response times from the first-order model are generated from a pair of Linear Ballistic Accumulators (LBA; Brown \& Heathcote, 2008) which race to predict a change or no-change response. In the second-order model, pairs of LBA's race at each possible change location. Consequently, we additionally consider whether each location is processed in parallel or in serial. A further development of our approach is that it allows us to differentiate whether the second-order integration of information considers only the maximum signal strength, terminating after a decision on that channel alone (i.e., using a self-terminating stopping rule) or whether more than one channel is considered (i.e., perhaps exhaustively checking the outcome of all channels). To assess this distinction, we consider multiple versions of the change detection task: one in which only a single change needs to be detected (an OR task) and one in which changes at all locations must be detected (an AND task). In both types of decision task, we further contrast redundant changes with changes at a single location to characterize the workload capacity of the system (Townsend et al., 2012; Townsend \& Nozawa, 1995; Townsend \& Wenger, 2004) along with the processing architecture. 
Wilken and $\mathrm{Ma}$ (2004) contrasted both first- and second-order signal detection models with a high threshold model (cf., Swets, 1961; Swets, Tanner, \& Birdsall, 1961; Tanner \& Swets, 1954a, 1954b). High threshold models are similar to slot-based models as they assume that items are either encoded into a limited number of available "slots" or not encoded at all (Cowan, 2001), in which case the participant guesses. Across a number of conditions varying stimulus type, Wilken and Ma (2004) found that hit rate decreased and false alarms increased as a function of increasing set size. The ROCs from their experiments were curvilinear, ruling out a high threshold model, but were equivocal in support for a first- or second-order model. Further, $\chi^{2}$ estimates favoured a second-order model in some instances but favoured the pooled model in other conditions.

For the signal detection models, Wilken and Ma (2004) assumed that participants monitored noisy information from a number of different locations (modelled as a Gaussian random variable). Only the absolute difference of the change was considered important, and if an item changes, the signal in the corresponding channel increases. Their first-order model, termed the Sum of Absolute Differences model (SAD) model, assumed that the participant sums the change strength signal across all locations. If this summed signal exceeds a decision threshold, a change response is emitted. When a change is present (in at least one location), the average value of the pooled signal is increased relative to when a change is absent. The effect of increasing the number of distractors causes an additional increase in the overall mean and variance of both the change present and change absent distributions. False alarms can occur in this context due to the variability in the change absent strength distribution; that is, the pooled signal can exceed the change threshold on the basis of noise alone. In their second-order model, Wilken and Ma (2004) assumed that a participant monitors the noisy change signal from a number of different locations independently. If any of these signals exceed their respective thresholds then a change response is emitted. This model is equivalent to Shaw's (1980) independent decisions model. Wilken and Ma term this model the maximum absolute difference model (MAD); somewhat confusingly, this model is equivalent to the minimum evidence integration rule in signal detection theory (Macmillan \& Creelman, 2004; see Figure 1, bottom right panel). ${ }^{1}$

To understand how these integration rules are instantiated in our modeling framework, it is useful to consider how a variable representation gives rise to hit and false alarm rates. The top panel of Figure 1 shows a two dimensional signal detection representation. The circles centered at the origin are the iso-probability contours of a bivariate normal density with a mean of $(0,0)$. This distribution represents the variable strength when there are no changes in the display. By contrast, the second distribution (in the top right) represents the variable strength when there are changes in both locations; the mean of this distribution is $\left(d_{x}^{\prime}, d_{y}^{\prime}\right)$ where $d^{\prime}$ is the difference between the means at each location. It is evident that if separate freely-varying thresholds are established on each dimension, the bivariate representation is equivalent to Wilken and Ma's (2004) SAD model. The SAD model, in

\footnotetext{
${ }^{1}$ The difference in terminology likely arises because Wilken and Ma (2004) emphasize the strength of the signal; whereas Macmillan and Creelman (2004) emphasize the amount of evidence required to make a decision. Hence, a maximum strength rule is equivalent to a minimum evidence rule. In this paper, we use the terminology which emphasizes evidence since it corresponds more readily to our discussion of response time.
} 

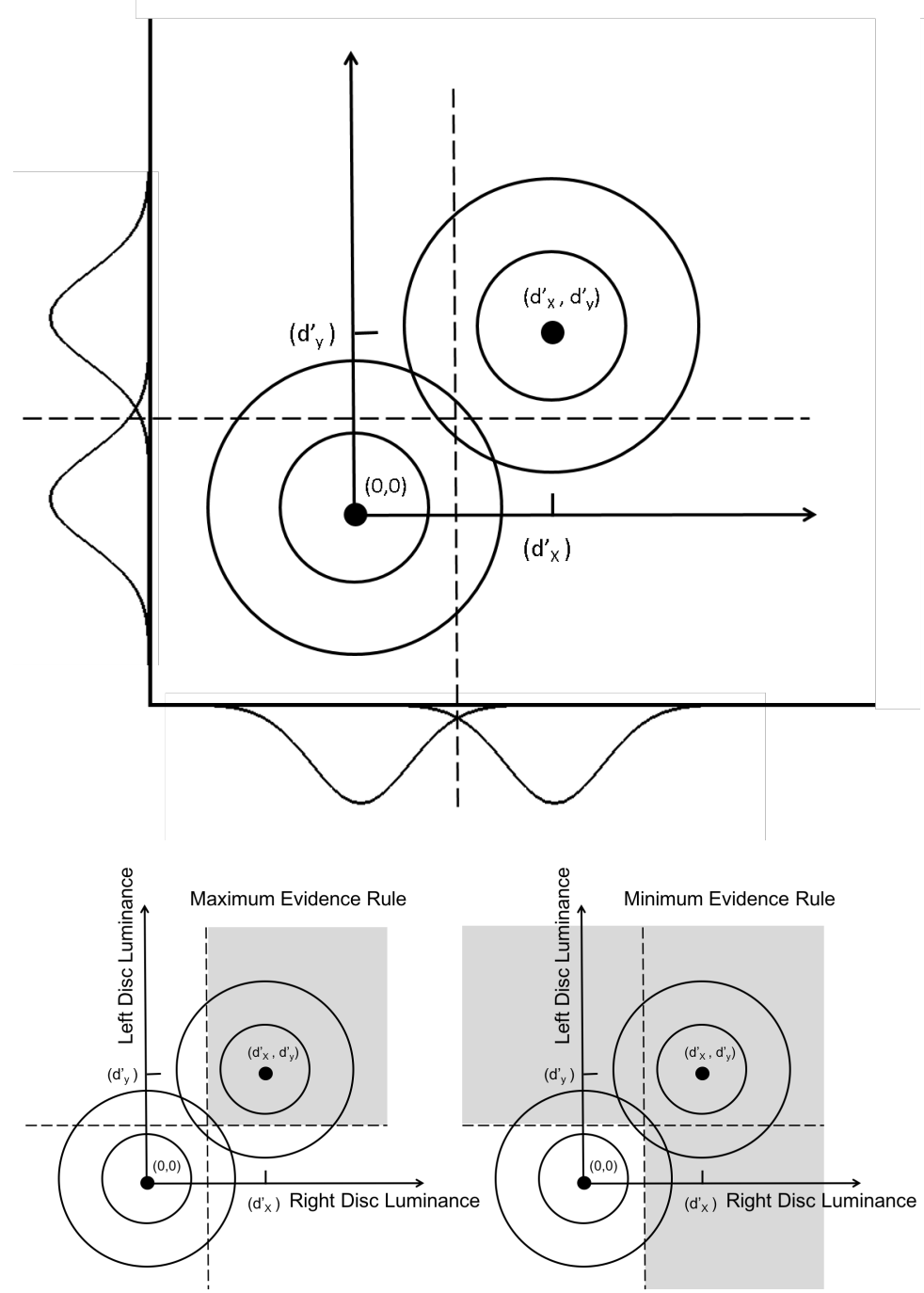

Figure 1. Top panel: A two dimensional signal detection representation for a task requiring detection of a change in luminance in one of two locations. The point $(0,0)$ represents the variable strength at the point of origin and the point $\left(d_{x}^{\prime}, d_{y}^{\prime}\right)$ represents the variable strength when there are changes in both locations. Circles surrounding these points represent isoprobability contours of a bivariate normal density. The dotted line represent decision thresholds. Bottom left panel: The maximum evidence integration rule (Macmillan \& Creelman, 2004). Bottom right panel: The minimum evidence integration rule (Macmillan \& Creelman, 2004). The shaded regions represent the area which must be integrated to determine the evidence for a change decision. Note: The minimum evidence integration rule is equivalent to Wilken and Ma's (2004) maximum strength rule. 
effect, samples a vector of change strengths from the model shown in Figure 1, sums the values on each dimension, and compares this to a single threshold. The integration within each region of the space in Figure 1 can be made equivalent to comparing the sum to a single threshold. That is, without further empirical constraint, you cannot tell these models apart using accuracy or choice probability.

The univariate marginal distributions shown in Figure 1 (top panel) represent the strength of change in each location independently. In Wilken and Ma's (2004) MAD model, separate decision criteria are established along each location. Change strengths are sampled from each independent distribution and compared to the individual thresholds. This is equivalent to integration using a minimum evidence rule (see Figure 1, bottom right).

When applied to change detection (as for example in Wilken \& Ma, 2004), the evidence signal is modeled as a difference between the study and test arrays. The differencing approach was initiated by Hefner (1958) as a method of scaling differences between dimensions. Hefner assumed that each study and test value was drawn from a normal distribution and that the difference between these values was compared to a threshold. Sorkin (1962) extended this approach to same-different auditory judgments. In Sorkin's model, differences were represented both above and below zero such that the direction of the change was also available as part of the representation. That is, two criteria were applied depending on whether the test tone was incremented or decremented from the study tone. A less general but potentially more parsimonious account of the change detection signal is to represent the absolute change between the study and the test arrays. Such a representation can be modeled using a folded-normal distribution (Leone, Nelson, \& Nottingham, 1961). This is the approach taken in the current paper.

Let the signal at each presentation location, $L$, be represented by a normally distributed random variable capturing the idea that the perceptual signal from each location is noisy:

$$
L_{i} \sim N\left(\mu_{L_{i}}, \sigma_{L_{i}}\right)
$$

where $\mu_{L_{i}}$ and $\sigma_{L_{i}}$ are the mean and standard deviation of the perceptual distributions, respectively. The subscript $i$ denotes whether the presentation of the stimulus at that location was in the memory array or the test array (e.g., time 1 or time 2). For each location, we assume that the representation of the change signal is provided by the distribution of the absolute differences at time 1 and time $2,|Z|=\left|L_{1}-L_{2}\right|$. We model this as a folded-Normal distribution:

$$
|Z| \sim \text { folded- } N\left(\left|\mu_{L_{1}}-\mu_{L_{2}}\right|, \sqrt{\sigma_{L_{1}}^{2}+\sigma_{L_{2}}^{2}}\right)
$$

In the following, we implement both the minimum evidence model and the pooled model, along with a number of other plausible variants, using either independent foldednormal distributions at each location or using a joint multivariate folded-normal distribution. In order to differentiate these explanations, we generalize the models to the time domain and design experiments that contrast the response time predictions of the models. While Dai, Versfeld, and Green (1996) showed that a model assuming independence was optimal when there was a fixed set of possible change detection stimuli and when the test display was not correlated with the study display, when a test change varied as a increment 
or decrement of the study display, a pooled differencing model is optimal. However, individual performance often falls short of these optimal strategies (Noreen, 1981) and as such it is not clear whether a first- or second-order model will best describe participant performance in the current paradigm. We now turn to a more detailed consideration of how reaction time can be used to effectively differentiate first- and second-order integration models.

\section{Using reaction time to differentiate integration models}

Few models have considered the time course of change detection, and none have contrasted the predictions of different models of integration for response time. Donkin, Nosofsky, Gold, and Shiffrin (2013) accounted jointly for choice probabilities as well as RTs using the LBA model. In this work, Donkin et al. (2013) developed different "front-end"2 models to compare slot-based and resource-based accounts of change detection (discussed in further detail below). Lilburn (2016) used a diffusion model to characterize decision processes while comparing near-threshold orientation discrimination and change detection, and Smith (2016) has modeled RTs in a closely-related continuous report task using a circular diffusion process. These approaches each either assume that the information which drives decision-making is accumulated in a single decision-making channel or circumvent the need to explicitly model decision-making architecture by only requiring the retrieval of a single item.

When we consider how change detection decisions play out over time, the question of first- and second-order models necessarily becomes more complicated. First, let us consider second-order models. In this class of models the signal strength representing a change for each location is compared to an individual criterion, and if the strength of the variable exceeds the criterion, then a target present response is made. Importantly, decisions are made based on each location separately and independently. Let us consider the task we use presently, in which a participant must detect a change in luminance in either, or both, of two discs present in the display (see Figure 3). If we consider second-order models in their general form, we can see that independence over time can be thought of in two ways. One may process the discs simultaneously but in independent parallel channels, or one may process the discs one at a time (i.e., in serial) in independent channels.

Returning to Figure 1, evidence from each disc can be integrated in two ways within each of these architectures. It may be that evidence from both discs is integrated in order to make a decision (i.e., a maximum evidence rule). Alternatively, evidence from only one disc may be used to make a decision (i.e., a minimum evidence rule). The use of a minimum or maximum evidence integration rule is somewhat independent of whether the integration is taken over the joint distribution (as in the pooled model) or in each of the marginal distributions independently. If the latter, then the minimum and maximum integration rules can be thought of as mapping on to self-terminating and exhaustive decision rules, respectively. For example, a parallel model with a minimum integration is most naturally related to a parallel self-terminating model. A parallel self-terminating model is logically consistent with a second-order model in its general form. Conversely, a parallel model with

\footnotetext{
${ }^{2}$ We use the term "front-end" model to refer to the set of processes, mechanisms, or algorithms that are used to derive the drift rate of the evidence accumulation process. These drift rates are then used to drive a "back-end" model which predicts the response time and choice for that trial; in the present example and in our paper, the backend model is the LBA or sets of LBA's combined using logical rules.
} 
a maximum integration rule is most conceptually similar to a parallel exhaustive model. In this model, RT is determined by the slowest of the decision channels, since both discs must be processed before a decision can be made. This represents a more conservative, but nonetheless independent, decision-making process.

A similar mapping applies in the case of the serial model. In a serial self-terminating model, if a decision can be made after processing the first location, then the process terminates. If a decision cannot be made after processing the first location, then the process continues to the second location. In a serial exhaustive model, completion of processing at the first location is then necessarily followed by the processing of the second location, again representing an independent, albeit conservative decision-making process.

For first-order models, evidence from each disc is summed or pooled into a single source. A first-order model would be equivalent to a coactive model where information from both locations is pooled into a single decision-making channel. Although this pooling results in a single decision-making channel, this class of models can also incorporate both evidence rules. The information which drives a decision can be either integrated using the maximum evidence rule (bottom left panel of Figure 1) or using the minimum evidence rule (bottom right panel of Figure 1). We term these models the coactive-max model and the coactive-min model, respectively.

Table 1

Summary of candidate models, including architecture and decision-rules, corresponding integration rule, and task to which the model is most relevant

\begin{tabular}{lccc}
\hline Model/Decision Rule & Architecture & Integration Rule & Task \\
\hline MAD $^{\mathrm{a}}$ & Coactive & Minimum Evidence & OR \\
SAD $^{\mathrm{b}}$ & Coactive & Maximum Evidence & AND \\
Self-terminating & Parallel & Minimum Evidence & OR \\
Exhaustive & Parallel & Maximum Evidence & AND \\
Self-terminating & Serial & Minimum Evidence & OR \\
Exhaustive & Serial & Maximum Evidence & AND \\
MAX Model & Single Channel & - & OR \\
MAX-first, Self-Terminating & Serial & - & AND \\
\hline
\end{tabular}

${ }^{\mathrm{a}} \mathrm{MAD}=$ Maximum Absolute Differences model.

${ }^{\mathrm{b}} \mathrm{SAD}=$ Sum of Absolute Differences.

We contrast this set of models, tabulated in Table 1, with two additional models that capture the idea that rather than integrating across each location, a pre-attentive parallel process is used to identify the strongest change signal in the display, which in turn drives the decision process. In the "MAX" model, there is a single channel decision channel in which only the signal with the maximum strength contributes to the decision. For accuracy or detection probabilities, a parallel self-terminating model is equivalent to a MAX model (Koopman, 1956); however, for the RTs, only the strongest location is actually processed. Hence, the MAX model RT predictions may be distinct from the parallel model, in which the weaker channel could also influence the response time. The MAX model predictions may also be different from the coactive-min model, in which the weaker channel is integrated into the change signal. When processing is exhaustive, as in an AND task, we 
considered a novel variant of the MAX model in which processing is serial with the location of highest strength prioritised; we term this model the MAX-first model. The extent of the qualitative difference between the MAX model, the parallel self-terminating model, and the coactive model is unclear. We next demonstrate that these models do, in fact, make similar qualitative predictions for response time within the OR task but can be differentiated by comparing the predictions across the OR and the AND tasks.

Logical rules applied to the detection of changes. The evidence rates (i.e., drift rates for the "change" and "no change" accumulators) are generated by integrating the folded normal change distribution with respect to the relevant decision rule. For the maximum evidence rule (Macmillan \& Creelman, 2004), this involves integrating over the region of space where both dimensions have changed (as shown previously in Figure 1). For the minimum evidence rule, this involves integrating over the region of space where only one dimension has changed (see Figure 1). For the coactive and single channel models, there is a single LBA pair; the decision and decision time are given by the faster of the two accumulators. For the MAX-first model, drift rates for both locations are calculated but only the maximum change drift rate is used. When processing is exhaustive, the MAX-first model processes the location with the strongest evidence first and then shifts to processing the next location if a decision could not be made on the first location.

For the serial and parallel models, we assumed that individuals make separate decisions along each dimension. In the current Experiment 1, where there are two locations, this gives four accumulators: one representing "change" and one representing "no change" for each of the two locations. In a parallel self-terminating model, the total decision time is the minimum of the time taken to make a decision at each location. For a parallel exhaustive model, the decision time is the maximum of the time it takes to make a decision at each location. For a serial self-terminating model, the decision time is the time taken for the first accumulator pair to complete, providing a decision can be logically made. If a decision cannot be logically made, then the second dimension is processed, and the decision time is the sum of time taken for each pair to reach a decision. The decision time in a serial exhaustive process is the sum of the time it takes each accumulator pair to reach a decision. A schematic illustration of the models is shown in Figure 2. 


\section{Differentiating response time architectures}

Each of the models in Table 1 and Figure 2 can be thought of as a parallel or serial or single-channel/coactive architecture. In most standard designs, these models mimic each other making them impossible to differentiate (Townsend, 1990a). For example, the observation that mean RT increases with set size in visual tasks is often taken to infer serial processing, but a limited capacity parallel model, which slows its rate of processing as the numbers of items increases, makes this same prediction (Little, Eidels, Houpt, \& Yang, 2017; Townsend, 1972; Townsend \& Ashby, 1983; Wenger \& Townsend, 2000). In the current study, we make use of the double factorial paradigm, which allows us to use Systems Factorial Technology to compute a number of non-parametric measures for which the preceding models make qualitatively unique predictions (Little, Altieri, et al., 2017; Townsend \& Nozawa, 1995).

Our novel change detection variant of the double factorial design (cf. Fifić et al., 2010) is shown in Figure 3. Following our recent work examining the categorization of discs varying in luminance in two different locations (Blunden, Howe, \& Little, 2020), participants were presented with a memory and probe array, both containing two luminance discs separated in space. In Experiment 1, participants were asked to respond "YES" if any of the discs changed (in the OR task) or alternatively to respond "YES" only if both items have changed (in the AND task). The double factorial design combines two experimental manipulations: one affecting workload - in which two changes are presented instead of one - and one affecting within channel processing speed - changes can be easy or hard to detect. ${ }^{3}$

To manipulate workload, we systematically varied the number of changes that could occur on any given trial. That is, on each trial there were either no changes, one change, or two changes. To manipulate processing speed, we varied the magnitude of each change. Changes could either be high salience $(\mathrm{H})$ or low salience $(\mathrm{L})$. The orthogonal combination of these two manipulations resulted in nine unique types of changes across two locations (see Figure 3). We assume that a high magnitude change provides a stronger change signal than does a low magnitude change consequently resulting in faster and more accurate detection. A stimulus which comprises two high salience changes (e.g., stimulus HH in Figure 3) will in turn involve a faster comparison process than a stimulus with one high salience and one low salience change (e.g., stimulus HL). Hence, stimulus dimensional values of either high discriminability $(\mathrm{H})$ or low discriminability $(\mathrm{L})$ combine to form four stimuli which are defined by their relative detection difficulty: HH, HL, LH, and LL.

The double factorial paradigm allows us to compare models using a number of interaction contrasts. First, the mean interaction contrast (MIC) is computed as follows:

$$
M I C=\left(\overline{R T}{ }_{L L}-\overline{R T} T_{L H}\right)-\left(\overline{R T} T_{H L}-\overline{R T} T_{H H}\right)
$$

\footnotetext{
${ }^{3}$ The double factorial design as originally introduced in Townsend and Nozawa (1995) was concerned with the simple detection of elements in different locations. The manipulation of the number of elements that were displayed was consequently a true manipulation of workload. For change detection, we similarly can manipulate workload by varying whether one target or two targets are presented; however, we can also examine efficiency when the number of targets remains the same but when the number of changes varies from one or two. For simplicity, we will use the term "workload" to refer to both of these cases and will clarify when the difference becomes important (cf. Little, Eidels, Fific, \& Wang, 2015, 2018). For instance, in Experiment 2, we compare performance between redundant changes and a separate condition in which only a single target location was presented.
} 

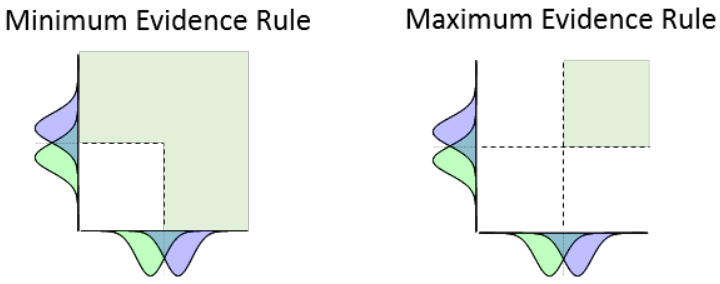

$$
\begin{aligned}
& v_{\text {Change }}^{\text {Coactive }}{ }^{\text {in }}=1-\int_{-\infty}^{d b_{x}} \int_{-\infty}^{d b_{y}} \text { folded }-N(x, y ; \mathrm{M}, \Sigma) d x, d y \\
& v_{\text {Change }}^{\text {Coactive max }}=\int_{d b_{x}}^{\infty} \int_{d b_{y}}^{\infty} \text { folded }-N(x, y ; \mathrm{M}, \Sigma) d x, d y \\
& \mathbf{v}_{\text {Change }}^{\text {Parallel }}=\mathbf{v}_{\text {Change }}^{\text {Serial }}=\mathbf{v}_{\text {Change }}^{\text {MAX first }}=\left[\begin{array}{l}
v_{x}=\int_{d b_{x}}^{\infty} \text { folded }-N\left(x ; \mu_{|L x 1-L x z|}, \sigma_{|L x 1-L x 2|}\right) d x \\
v_{y}=\int_{d b_{x}}^{\infty} \text { folded }-N\left(y ; \mu_{|L y 1-L y 2|}, \sigma_{|L y 1-L y 2|}\right) d x
\end{array}\right] \\
& v_{\text {Change }}^{\mathrm{MAX}}=\max \left(v_{x}, v_{y}\right)
\end{aligned}
$$

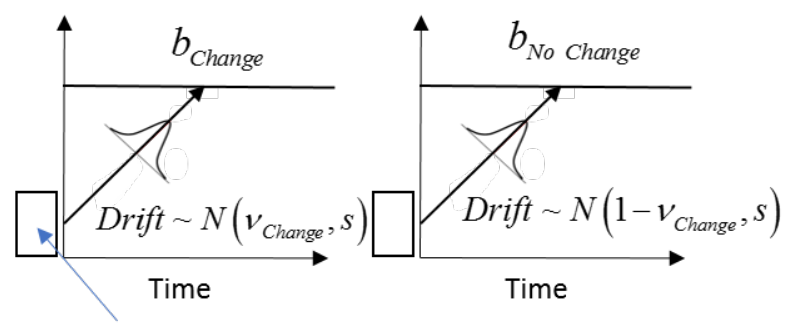

$$
\text { start point } \sim U(0, A)
$$

Figure 2. Top panel: Minimum and maximum evidence rules. Middle panel: Equations showing how drift rate is derived from the integration rules for each of the models. Bottom panel: Schematic illustration of the Linear Ballistic Accumulator. 
Coactive, MAX

Decision Time $(D T) \sim \operatorname{LBA}\left(\mathbf{v}_{\text {Change }}, A, b_{\text {Change }}, b_{\text {No Change }}\right)$

\section{Left Change \\ Right Change}

Total $\mathrm{RT}=$ Decision Time $+t 0$

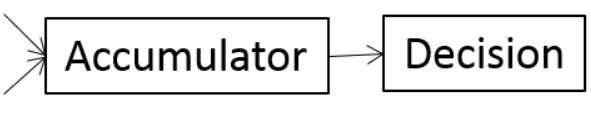

\section{Parallel Self-Terminating}

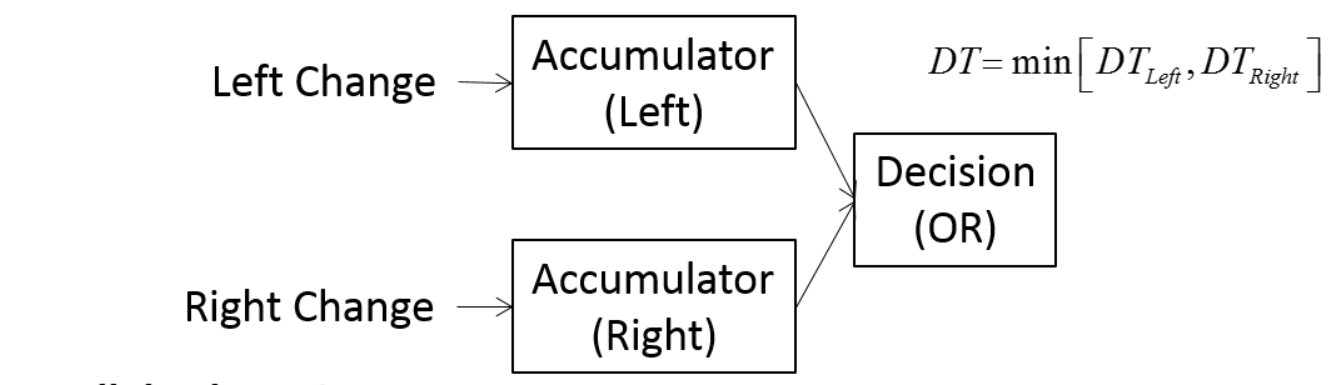

\section{Parallel Exhaustive}

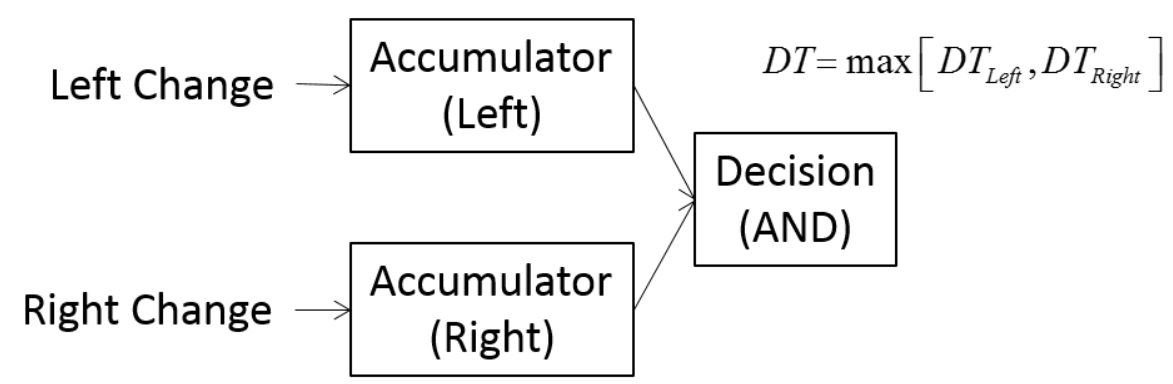

\section{Serial Self-Terminating, MAX first}

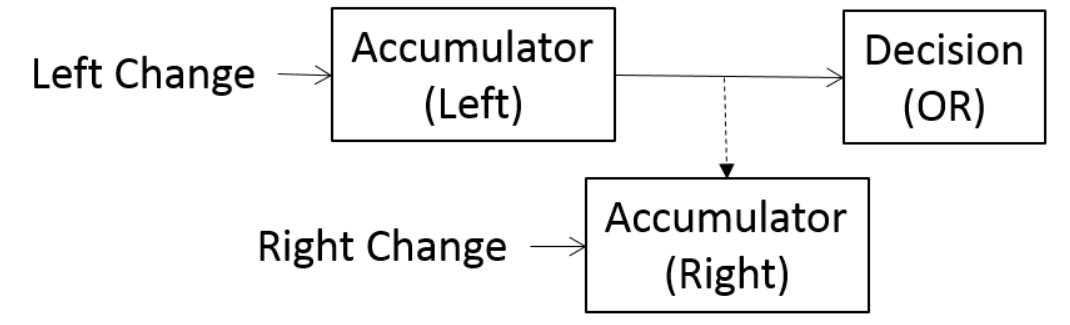

\section{Serial Exhaustive}

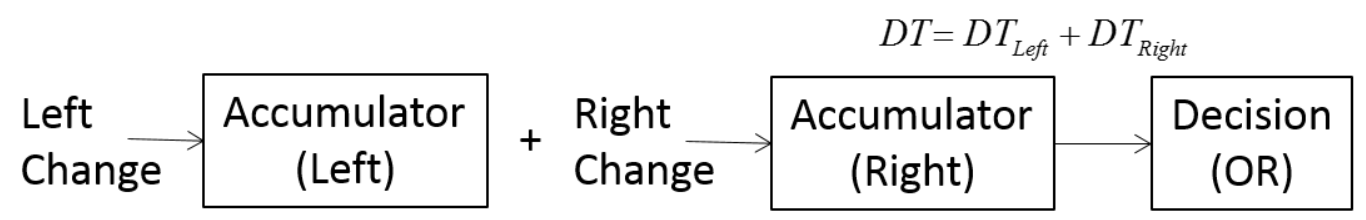

Figure 2. Schematic illustration of how the linear ballistic accumulators are combined to detect a change in the left hand channel for each of the different models. 


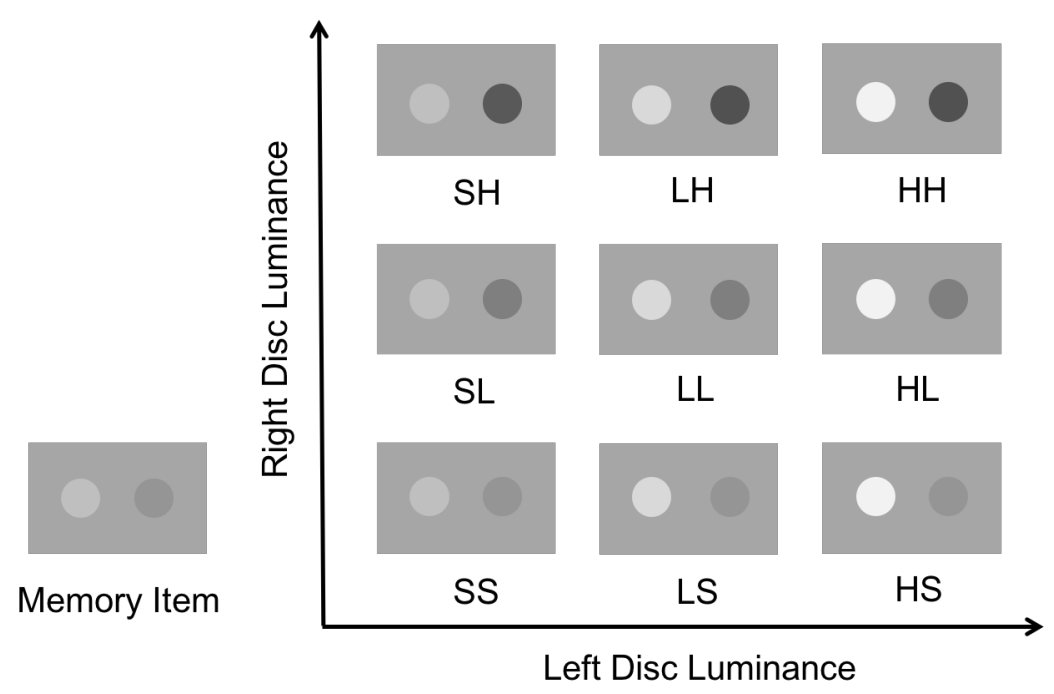

Figure 3. Schematic of stimulus space with an example memory array and the corresponding trial types. $\mathrm{S}$ denotes same or no change; $\mathrm{L}$ denotes low magnitude change; $\mathrm{H}$ denotes high magnitude change. These are combined to form nine possible change types. For example, LH indicates a low magnitude change in the left channel and a high magnitude change in the right. In practice, any item from the nine item stimulus space could appear as either the memory item or the probe item.

where $\overline{R T} T_{L L}$ is the mean RT from LL change condition. An additive pattern $(M I C=0)$ is evidence for a serial process; this is true for both the self-terminating and exhaustive cases. An under-additive MIC $(M I C<0)$ is indicative of a parallel exhaustive model, and an over-additive MIC $(M I C>0)$ is predicted by both a parallel self-terminating model and a coactive model. On its own the MIC provides a simple measure of processing architecture, but when combined with the full RT distribution (the survivor interaction contrast, SIC, which is outlined below), the MIC further provides a useful means of statistically assessing the significance of interactions shown at the distributional level.

The SIC is defined as follows:

$$
S I C(t)=\left[S_{L L}(t)-S_{L H}(t)\right]-\left[S_{H L}(t)-S_{H H}(t)\right]
$$

where $S(t)$ is the survivor function for a specific item condition. The SIC provides a more diagnostic contrast which utilizes the entire RT distribution. The SICs for serial, parallel, and coactive models, as well as the relevant stopping rules, are shown in Figure 4.

Consider the processing of a double change trial, such as HH. While both serial selfterminating and serial exhaustive models predict an $M I C=0$, the SIC functions of these model classes have very different forms. Serial self-terminating models predict an entirely flat curve (i.e., every time point is equal to zero) whereas serial exhaustive models predict an initially negative function which becomes positive, with the entire function integrating to zero (since the integral of the survivor function equals the mean RT, the integral of the SIC equals the MIC; Townsend, 1990b). For serial self-terminating models, only a single location needs to be processed before a change decision can be made and only the decision 

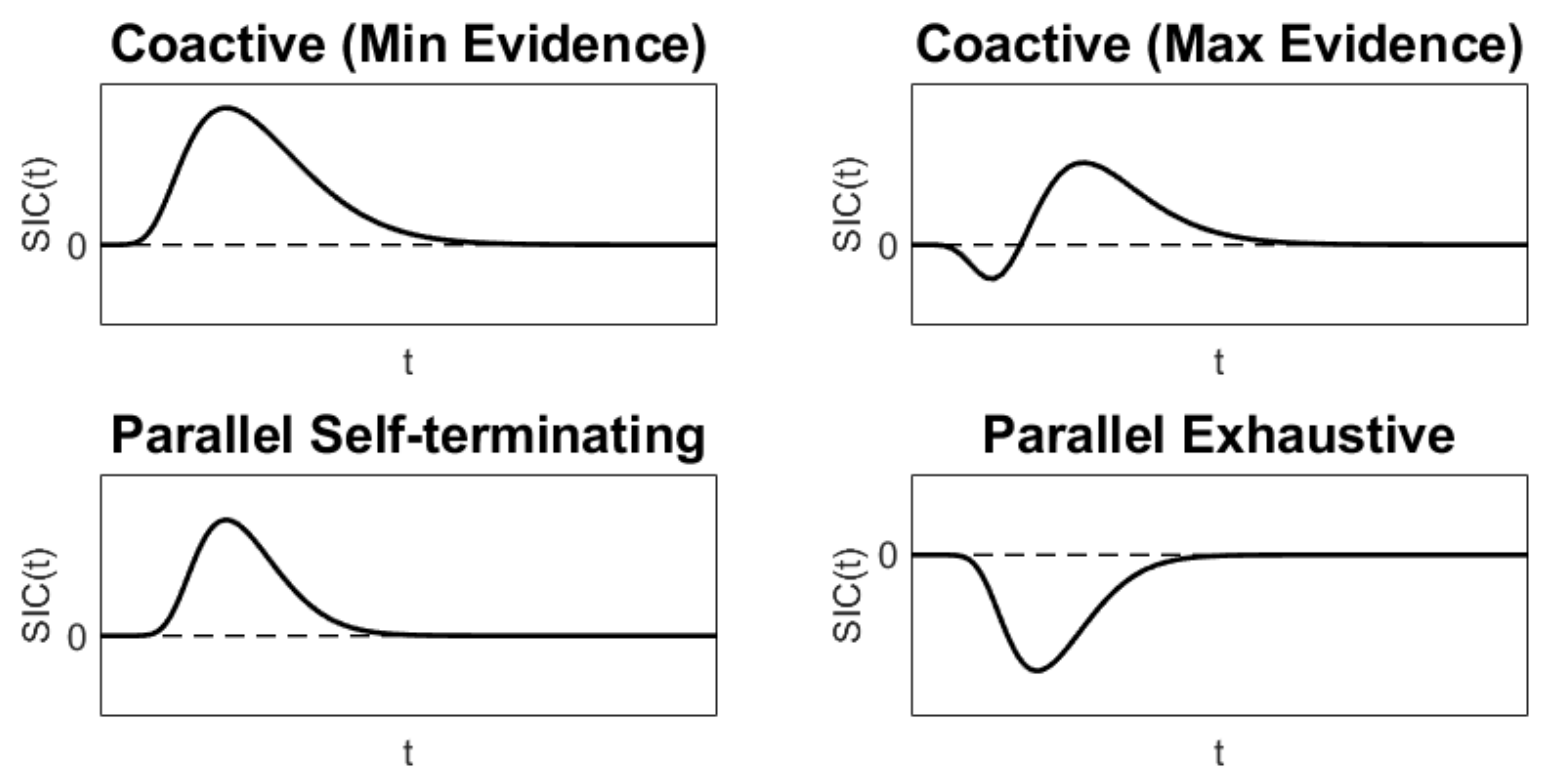

Serial Self-terminating

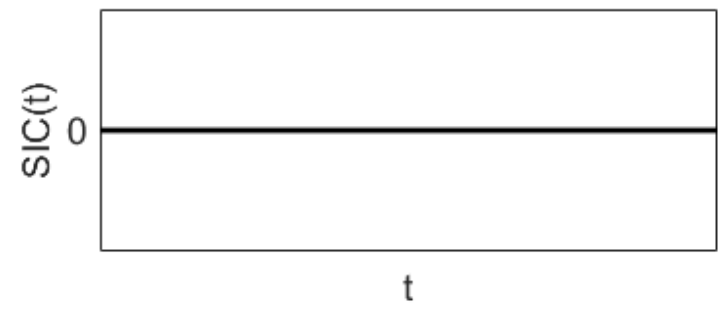

Serial Exhaustive
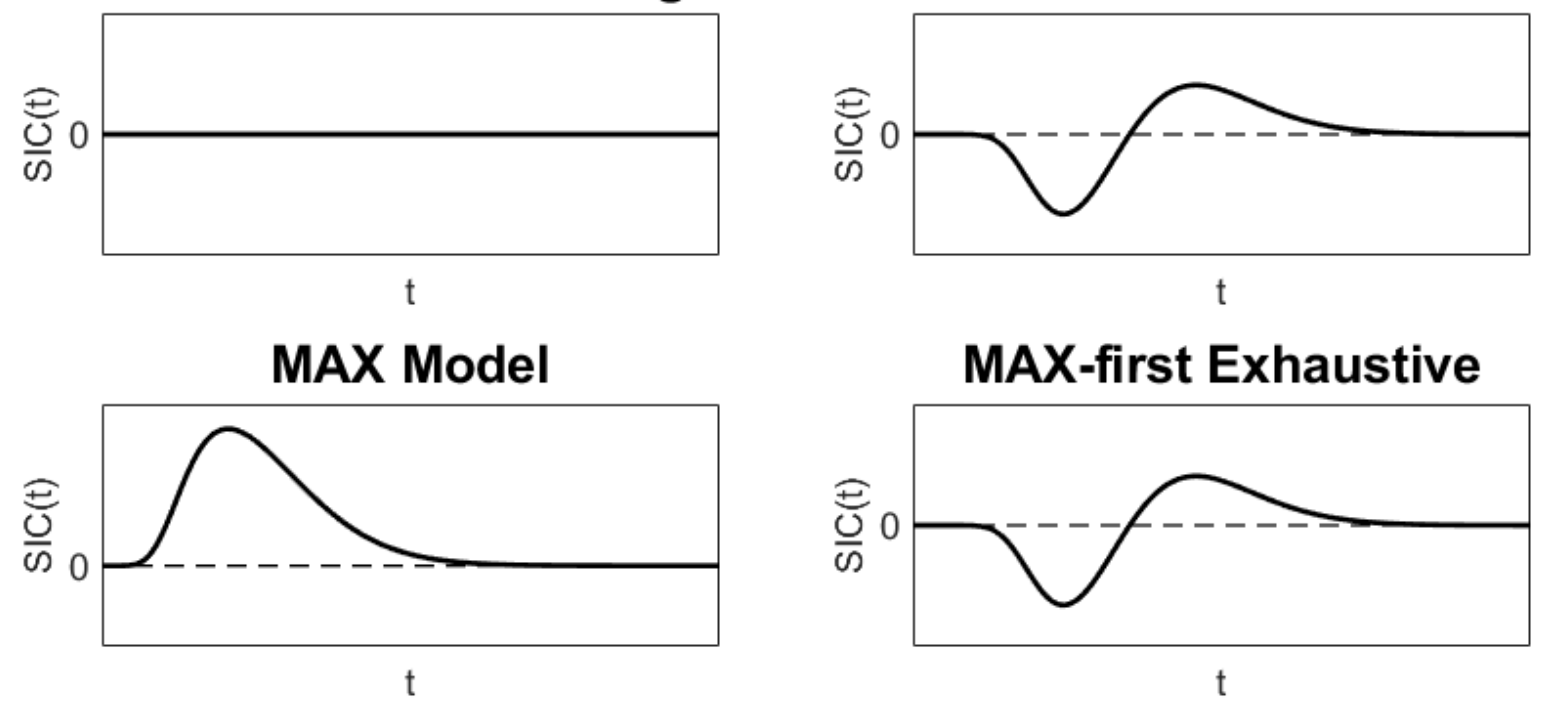

Figure 4. Survivor interaction contrast predictions for the coactive, parallel, serial, and MAX models 
time for that location contributes to the overall RT (see Figure 2, serial self-terminating). In the SIC, the survivor functions for $\mathrm{HH}$ and HL cancel out as do the survivor functions of LH and LL resulting in a curve which equals 0 for all $t$. By contrast, for serial exhaustive models, the overall RT is given by the sum of the times at each location (see Figure 2, serial exhaustive). For the SIC, an additive pattern arises, as both LH and HL items will show some slowing relative the to $\mathrm{HH}$ item due to their lower discriminability on one of the dimensions. The increase of RT for the LL item compared to the HH item is the sum of the individual sources of slowing.

For a parallel self-terminating model, the decision time is determined by the minimum processing time at each location (see Figure 2, parallel self-terminating). The implication is that HH, HL, and LH will all be processed faster than the LL item, resulting in a positive SIC. By contrast, the decision time in a parallel exhaustive models is determined by the maximum processing time at each location (see Figure 2, parallel exhaustive). Consequently, parallel exhaustive models predict an entirely negative function since the HL, LH, and LL change trials will have larger maximum times than the $\mathrm{HH}$ change trials.

The coactive-max model predicts an initial negativity in the SIC function followed by positivity at longer $t$. A key characteristic of coactive models is that the positive portion of the function is greater than the negative portion; the SIC of a coactive model therefore does not integrate to zero but rather integrates to a positive value. This over-additive pattern arises from a number of coactive models (including random walk models, accumulator models in which the rates of both channels are summed, and interactive models with complete facilitation between channels) and has been confirmed using mathematical proofs and simulations (Eidels, Houpt, Altieri, Pei, \& Townsend, 2011; Fifić et al., 2010, 2008; Houpt \& Townsend, 2011; Townsend \& Nozawa, 1995). By contrast, the coactive-min model predicts a completely positive SIC. We confirmed this prediction across a number of parameter settings by simulating the predictions of our bivariate folded-normal model coupled with an LBA. Finally, the MAX model also predicts a completely positive SIC function, while the MAX-first model predicts an SIC function which resembles the prediction of the serial processing model.

The MIC and SIC predictions are non-parametric in that they do not depend on the particular form of the RT distributions and can be computed directly from the data. For example, the SIC shape of the coactive-max model applies regardless of whether the true RT generating model is an LBA or drift diffusion model (Ratcliff, 1978). As shown in Figure 2, several of the models can be distinguished by their qualitative contrasts. The predictions become more powerful when considered across both OR and AND tasks.

In the OR task, although exhaustive and maximum evidence coactivity models are possible, it is likely that processing will self-terminate. This follows from the idea that observers act to conserve mental effort (e.g. Hull's "law of less work"; Hull, 1943; Kool, McGuire, Rosen, \& Botvinick, 2010). Indeed, across many perceptual decision-making experiments, we have not observed exhaustive processing when the experiment allowed for self-termination (Blunden et al., 2020, 2015; Chang, Little, \& Yang, 2016; Cheng, McCarthy, Wang, Palmeri, \& Little, 2018; Fifić et al., 2010; Little et al., 2011, 2013; Moneer et al., 2016; C.-T. Yang, Altieri, \& Little, 2018; C.-T. Yang, Little, \& Hsu, 2014; H. Yang, Little, Eidels, $\&$ Townsend, 2019). Further, there is also evidence that participants will attempt to apply self-terminating processing even in exhaustive tasks which do not permit self-termination 
without error (Bushmakin, Eidels, \& Heathcote, 2015; Howard, Garrett, Little, Townsend, \& Eidels, 2020). Conversely, in an AND task, exhaustive processing is dictated by the task, and we expect that observers will apply a max evidence or exhaustive processing model for the double change items, although participants may self-terminate where logical to do so for single change items. Below we will fit each of the models to each of the OR and AND tasks, but taken together, the most relevant predictions for the OR task are shown in the left-column of Figure 2 while the most relevant predictions for the AND task are shown on the right. Each row then presents a pair of predictions from a different model class for the OR task and the AND task. Across both tasks, each model class makes a unique set of predictions.

\section{Previous work using SFT to investigate change detection}

Similar work by C.-T. Yang (2011) as well as C.-T. Yang, Hsu, and Yeh (2011) and C.-T. Yang, Chang, and Wu (2013) has used SFT in the context of change detection. Unlike the current work, however, rather than investigating how information from multiple items is integrated, Yang and colleagues examined how various task manipulations affected attentional deployment and processing architecture. For example, C.-T. Yang et al. (2011) asked participants to detect contrast and orientation changes in a single Gabor patch. The experiment consisted of two conditions: equal and unequal salience changes. In the equal salience condition, changes to both dimensions were scaled such that discriminability of contrast and orientation changes were equated. In the unequal salience condition, the relative salience of changes to the two dimensions differed across participants. In the equal salience condition, performance was uniformly parallel and self-terminating, while in the unequal salience condition performance varied between serial and coactive.

Further, C.-T. Yang et al. (2013) found that the probability of a change occurring also affected the response architecture. In cases where spatial frequency changed more often than did orientation, the two changes were processed serially. However, when a change in either was equally likely, parallel processing was more likely to be adopted. This shift from serial to parallel occurred despite participants reporting that they were unaware of using information regarding the relative probability of a change to complete the task (see also Chang et al., 2016). Additionally, when there was a lack of cognitive resources, participants reverted to parallel processing even when relative change probability was not equated. While these studies provide further insight into how varying dimensional change probability and salience within a single object affects processing architecture, our question is more fundamental. In our study, we hold constant salience and change probability and instead look simply at the architecture of combining information from multiple locations in change detection decisions.

Thiele, Haaf, and Rouder (2017) examined processing architecture in a simultaneous vs sequential object comparison using an SFT design; the latter task can be considered a change detection task. For both tasks, using an AND design, they found that detection of changes for separable dimensions (orientation and size of a "screwhead") was consistent with serial processing. In a second experiment using digits, a within-dimension feature, there were a larger number of participants consistent with parallel processing. Here we additionally consider processing in an OR task along with a larger set of models. 


\section{Current Experiment}

In Experiment 1, we sought to diagnose the processing architecture of change detection decisions using two luminance discs of different polarity presented at different locations (see Figure 3). We limited the display size to two to avoid exceeding a potential item capacity limit (see e.g., Sewell, Lilburn, \& Smith, 2014). We investigated both an OR and an AND task. In the OR task, participants needed to detect any change in the probe array, whereas in the AND task, participants needed to verify that both items had changed. It is possible that participants may engage in different decision-making/integration strategies across these two tasks. To diagnose these strategies, we tested a wide variety of models consistent with both first- and second-order accounts which predict both choice probability and RT across the entire distribution. By characterizing the processing architecture of change detection decisions we answer Wilken and Ma's (2004) question of whether information integration is best described by a first- or second-order integration model.

We additionally considered the efficiency of detecting one change versus detecting two changes using the Systems Factorial Technology measure of workload capacity Townsend and Nozawa (1995). In short, we used performance on the single change targets to derive the prediction of a baseline unlimited capacity independent parallel processing model. This baseline is the expected minimum processing time in the OR condition and the expected maximum time in the AND condition. We then compare double target performance by taking the ratio of the double target processing time to the expected time, taking the integrated hazard function (or the reverse integrated hazard function in the case of the AND task) of both (Chechile, 2003, 2011; Townsend \& Eidels, 2011). This provides a baseline prediction which equals one across all $t$. Performance greater than one indicates better than parallel efficiency; performance less than one indicates worse than parallel efficiency. This measure is explained more fully in the Data Analysis section below and explored in more detail in Experiment 2; consequently, we defer our primary discussion of capacity until Experiment 2 but for comparison, we compute this measure as a secondary consideration in Experiment 1.

Our primary consideration in setting the frequency of each trial type was to collect an adequate number of RTs, within the allocated number of sessions, for each of the double target items to allow for accurate estimation of the response time distributions. The frequencies in the AND condition were the same as in the OR condition (see Table 2). We foreground that these choices have implications for the interpretation of our capacity analysis (Houpt, Blaha, McIntire, Havig, \& Townsend, 2014; Mordkoff \& Miller, 1993; Mordkoff \& Yantis, 1991), which we will address below, but will not affect the interpretation of the SIC analyses.

\section{Experiment 1}

\section{Method}

Participants. Twelve individuals with normal or corrected-to-normal vision (visual acuity of at least 20/25 as determined by a near-field Snellen eye chart; normal color vision as determined by Ishihara plates) from the University of Melbourne community participated in the study (OR condition: 5 males, 1 female; age range 25-30; AND condition: 3 males, 2 females, 1 undisclosed; age range 19-21, 3 undisclosed). Participants were recruited via 
advertising placed on notice boards within the Melbourne School of Psychological Sciences and through the school's online recruiting system. All participants were naïve to the purpose of the experiment. One participant from the OR condition and one participant from the AND condition did not complete all sessions and were excluded from the study. The remaining participants were labelled O1 - O5 (O denoting "OR") and A1 - A5 (A denoting "AND"). The AND condition was conducted after the OR condition. All participants were reimbursed $\$ 10$ per session. Testing was approved by the Melbourne Human Research Ethics Committee (Approval Number 1034866).

Apparatus and Stimuli. Stimuli were two discs subtending a visual angle of $1.91^{\circ}$ at $60 \mathrm{~cm}$ viewing distance presented on a gray background (RGB color space value [128 128 128]) with centers $11.34^{\circ}$ of visual angle to the left and right of fixation (center of the screen). Stimuli were presented on a CRT monitor with a resolution of $1280 \times 1024$ pixels with a refresh rate of $85 \mathrm{~Hz}$.

The disc on the left was always an increment relative to the background while the disc on the right was always a decrement. Each disc could take on one of three different luminance levels. In RGB color space these were [228 228 228; 192192 192; 156156 156] for the left disc and [40 40 40; 7676 76; 112112 112] for the right disc. These values were combined orthogonally to create a $3 \times 3$ matrix of possible stimuli presented in Figure 3. Both memory items and probe items were drawn from this display giving nine possible change types (see Figure 3 for an example of one memory item and probe set).

On a given trial, there could be no change, one change, or two changes between the memory and probe array. For change trials, luminance increased or decreased by one step (a low discriminability change) or two steps (a high discriminability change). For example, when considering only the left disc, a one-step change could have involved a change from the highest to the middle level or from lowest to the middle level. If the memory item was the middle level of luminance, the change could have been either incremented to the highest level or decremented to the lowest level for the probe item. A two-step change, however, could only involve a decrement from the highest to the lowest level and vice versa. In total, this gave 16 unique stimulus combinations for LL trials, eight unique stimulus combinations for HL and LH trials respectively, and four unique stimulus combinations for the HH item. Trial frequencies are shown in Figure 2. The experiment was conducted using Matlab and Psychophysics Toolbox (Brainard, 1997; Kleiner et al., 2007; Pelli, 1997); RTs were collected using a calibrated response time box (Li, Liang, Kleiner, \& Lu, 2010).

\section{Procedure}

The task was a one-shot change detection task with either an OR or an AND response rule. For the OR condition, participants were instructed to respond "YES" if either or both of the discs changed. For the AND condition, participants were instructed to respond "YES" only if both discs changed. Participants in the OR condition completed five sessions; participants in the AND condition completed six sessions. Sessions began with 15 practice trials, followed by two blocks of 220 trials each. Trials were randomized within each block. At the start of each session participants were shown a diagram of the stimulus space displaying all nine possible stimulus displays. Participants were given a short break every 74 trials. Each trial began with a fixation cross for $500 \mathrm{~ms}$, followed by the memory array for $1500 \mathrm{~ms}$. The memory array was then masked by two successive noise patches, 
each display for $500 \mathrm{~ms}$ with no gap, followed by a blank interval of $1000 \mathrm{~ms}$. Finally, the probe array was presented for $5000 \mathrm{~ms}$ or until a response was made.

Table 2

Trial frequencies per block and total presentation numbers for each trial type for Experiment 1 .

\begin{tabular}{lrrrrrrrrr}
\hline & \multicolumn{1}{c}{ Trial Types } \\
\cline { 2 - 10 } & \multicolumn{1}{c}{ Double Change Trials } & Single Change Trials \\
\cline { 2 - 10 } & LL & LH & HL & HH & HS & LS & SH & SL & SS \\
\hline Number of Unique Items per Block & 16 & 8 & 8 & 4 & 6 & 12 & 6 & 12 & 9 \\
Number of Repetitions per Block & 1 & 2 & 2 & 4 & 2 & 1 & 2 & 1 & 12 \\
Total per Experiment & 160 & 160 & 160 & 160 & 120 & 120 & 120 & 120 & 1080 \\
\hline
\end{tabular}

For the OR condition, upon presentation of the probe array, participants were asked whether the probe was the same as or different to the memory array. They responded "DIFFERENT" by pressing the right button on the RT Box and "SAME" by pressing the left button on the RT Box. For the AND condition, upon presentation of the probe array, participants were asked whether both objects were different. Participants responded "YES" by pressing the right button and "NO" by pressing the left button. Participants were then told whether their response was correct, incorrect, or too slow (in the event of taking longer than $5000 \mathrm{~ms}$ to respond). Feedback was presented for $1000 \mathrm{~ms}$. Participants were given feedback regarding their overall accuracy at the end of each session.

Data Analysis. Selective Influence. The SIC and MIC analyses of processing architecture rely on an assumption of effective selective influence (Dzhafarov, 2003; Schweickert et al., 2012; Townsend \& Schweickert, 1989). Selective influence implies that the experimental manipulation of salience in one location only affects processing at that location, leaving processing in the other locations unchanged. The manipulation should also be effective such that RTs for the high level channel should be shorter than the RTs for the low level channel. If this assumption holds, then the survivor functions will be ordered such that $S_{H H}(t) \leq S_{H L}(t) \approx S_{L H}(t) \leq S_{L L}(t)$ with the strict inequality holding for at least one time point (Townsend \& Nozawa, 1995). If this ordering is maintained, then the SIC and MIC can be interpreted to infer the processing architecture and stopping rule. We test this ordering using a series of Kolmogorov-Smirnoff (KS) tests comparing the ordering for the survivor functions for the following comparisons: $H H>H L, H H>L H, H L>L L$, and $L H>L L$, which we expect to be significant as these inequalities violate the expected survivor function ordering, and $H H<H L, H H<L H, H L<L L$, and $L H<L L$, which we expect to be non-significant because these inequalities are commensurate with the expected ordering if selective influence holds (Houpt et al., 2014).

Tests of MIC and SIC inference. We applied three inference techniques to assess the SIC pattern. First, we analysed the interaction effect (i.e., the MIC) using a Session $\times$ Left Change $\times$ Right Change ANOVA to assess whether the architecture (or processing strategy) changes across sessions and to assess whether the MIC is significantly underor over-additive. The interaction of Left Change $\times$ Right Change is a direct test of the significance of the MIC (Sternberg, 1969). Second, we tested the shape of the SIC curve by 
applying the $\mathrm{D}+$ and $\mathrm{D}$ - tests of the maximum and minimum SIC deflections as proposed by Houpt and Townsend (2010) and Houpt et al. (2014). To determine whether the negative and positive portions of the SICs were significantly different to zero, we used two onesided KS Tests from Houpt et al. (2014)'s SFT analysis package. Like the MIC, the null hypothesis for these statistics is $S I C(t)=0$ for all times $t$. A conservative significance level of $p<.05$ therefore biases the test toward retaining the null hypothesis (and therefore a bias toward and inference of serial processing). We therefore adopted a less conservative cut-off of $\alpha=0.33$. This value has been used in previous research (Blunden et al., 2020) and has shown to work well in model recovery tests using this statistic (Fox \& Houpt, 2016). Finally, we also constructed bootstrapped confidence intervals for the SIC to assess the uncertainty in the SIC.

Measuring Workload Capacity. The capacity coefficient is computed using the integrated hazard functions, $H(t)$, of the single and double targets. $H(t)$ gives the cumulative processing completed by the system until time, $t$. The hazard function is defined by the probability density function, $f(t)$, which gives the probability of observing a RT at time, $t$, divided by the survivor function, $S(t)$, which gives the probability that a process will finish after time, $t$ :

$$
H(t)=\int \frac{f(t)}{S(t)} d t=-\log (S(t))
$$

If we represent a change in the left position as $A$, a change in the right position as $B$, and a change in both positions as $A B$, then for the OR task, the capacity coefficient is defined as the ratio of the integrated hazard functions for the double target, $H_{A B}(\mathrm{t})$, over the sum of the two single target integrated hazard functions, $H_{A}(\mathrm{t})$ and $H_{B}(\mathrm{t})$. Because our single target conditions also contained an additional location which did not change, for clarity, we identify our single target conditions as, $H_{A} S(\mathrm{t})$ and $H_{B} S(\mathrm{t})$. As noted in Little et al. (2015) and Houpt and Little (2017), the inclusion of the "same" location can affect measurement of workload capacity (and even more so in the AND condition, see Howard et al., 2020). The problem for the AND task is that participants can shift attention to the non-target location, effectively self-terminating when a "same" stimulus is detected. This leads to a mis-estimation of capacity in the AND task (Howard et al., 2020). When this occurs, we expect there to be no difference between single targets of different salience. In the OR task, the SL and LS conditions were always slower than the SH and HS conditions, but this was not consistently the case in the AND task. Nonetheless, with that caveat in mind, the measure still provides an indication of performance relative to the unlimited capacity baseline model. Capacity in the OR task is computed as:

$$
C_{O R}(t)=\frac{H_{A B}(t)}{H_{A} S(t)+H_{B} S(t)}
$$

A system with unlimited capacity will produce a capacity coefficient equal to one across the entire time course of processing; $C_{O R}(t)=1$, whereas $C_{O R}(t)<1$ indicates limited capacity (worse than baseline performance for the redundant targets) and $C_{O R}(t)>$ 1 indicates supercapacity (better than baseline performance for the redundant targets).

Because the AND task requires exhaustive processing for the double targets, the baseline comparison is to an unlimited capacity, independent exhaustive parallel model. 
Consequently, the $C_{A N D}(t)$ requires use of the reverse integrated hazard function; $K(t)$ (Townsend \& Wenger, 2004). $K(t)$ can be defined as the log of $F(t)$ :

$$
K(t)=\log (F(t))
$$

Capacity for the AND case is defined as the ratio of the sum of the reverse integrated hazard functions for the single targets, $K_{A} S(\mathrm{t})$ and $K_{B} S(\mathrm{t})$, over the double target, $K_{A B}(\mathrm{t})$ :

$$
C(t)_{A N D}=\frac{K_{A}(t)+K_{B}(t)}{K_{A B}(t)}
$$

The use of the reverse integrated hazard function allows for $C_{A N D}(t)$ to have the same interpretation as $C_{O R}(t)$. That is, $C_{A N D}(t)=1$ indicates unlimited capacity, $C_{A N D}(\mathrm{t})<1$ indicates limited capacity, and $C_{A N D}(t)>1$ indicates super capacity.

To assess the capacity coefficient, we additionally computed two bounds which place upper and lower limits on extent to which the baseline model can predict capacity above and below $C(t)=1$. In the OR task, these bounds are the upper bound formed by the Race Model Inequality (Miller, 1982) and the Grice bound (Grice, Canham, \& Gwynne, 1984). For the AND task, the bounds are the upper and lower bounds defined by Colonius and Vorberg (1994). Exact mathematical forms of these bounds are given in Townsend and Eidels (2011). In essence, $C(t)$ values above the upper bound indicate extreme supercapacity whereas $C(t)$ values below the lower bound indicate extreme limited capacity. We also constructed bootstrapped confidence intervals on capacity to provide a measure of uncertainty in our estimates and analysed the significance of the capacity functions deviation from 1 using a $z$-test on the average capacity value across time (Houpt \& Little, 2017; Houpt \& Townsend, 2012).

\section{Results}

For the remaining participants, the first session was excluded as practice. Additionally, RTs less than $200 \mathrm{~ms}$ or greater than $3000 \mathrm{~ms}$ were excluded. In total, less than $3 \%$ of trials were removed using this method.

Mean correct RTs, mean error RTs, and error rates for the OR Task are presented in Table 3 and for the AND Task in Table 4. In the OR task, the error rate is consistently higher for the LS item condition than for any other item and somewhat higher for SL and LL compared to the remaining items. Looking at the single targets, SL and LS have consistently larger RTs than SH and LH.

\section{Tests of Selective Influence}

Figures B1 and B2, depicting the survivor functions for OR and AND tasks, respectively, are shown in Appendix B. The ordering of the double target mean RTs and survivor functions looks appropriate for all subjects except for O5, who has a smaller RT for LH compared to the other double target items, and A1, whose HL and LH survivor functions cross over the HH survivor function. Following Houpt et al. (2014), we analysed the stochastic ordering of the double target survivor functions using a series of KS tests (see Table ??). Participant A1 also performed near chance for the LL item. In addition to flagging participants $\mathrm{O} 5$ and $\mathrm{A} 1$, participant $\mathrm{O} 2$ also had a significant test for the $\mathrm{HH}>\mathrm{HL}$ comparison. 
Table 3

Observed Mean Correct and Error RTs (ms), and Error Rates for Individual Stimuli for each Participant in Experiment 1: OR Task

\begin{tabular}{llrrrrrrrrr}
\hline & & \multicolumn{10}{c}{ Item } \\
\hline Participant & Variable & \multicolumn{1}{c}{ HH } & HL & LH & LL & HS & LS & SH & SL & SS \\
\hline O1 & RT correct & 547 & 622 & 598 & 697 & 635 & 814 & 645 & 740 & 637 \\
& RT error & 1051 & 681 & 720 & 773 & 768 & 664 & 788 & 685 & 790 \\
& p(error) & .04 & .06 & .06 & .29 & .16 & .71 & .08 & .37 & .08 \\
O2 & RT correct & 572 & 592 & 673 & 793 & 587 & 826 & 681 & 956 & 773 \\
& RT error & 490 & - & - & 1304 & 2023 & 953 & 943 & 930 & 1171 \\
& p(error) & .01 & .00 & .00 & .07 & .01 & .37 & .02 & .17 & .03 \\
O3 & RT correct & 479 & 527 & 516 & 593 & 512 & 664 & 507 & 598 & 522 \\
& RT error & 764 & 628 & 494 & 563 & 671 & 563 & 646 & 544 & 657 \\
& p(error) & .01 & .07 & .02 & .30 & .09 & .54 & .05 & .39 & .15 \\
O4 & RT correct & 482 & 520 & 500 & 609 & 530 & 732 & 512 & 718 & 630 \\
& RT error & - & - & - & 658 & - & 646 & - & 600 & 924 \\
& p(error) & .00 & .00 & .00 & 0.01 & .00 & .37 & .00 & .09 & .02 \\
O5 & RT correct & 558 & 658 & 538 & 683 & 693 & 765 & 544 & 751 & 703 \\
& RT error & - & 1129 & 642 & 816 & 851 & 721 & 1406 & 849 & 1022 \\
& p(error) & .00 & .04 & .02 & .16 & .11 & .55 & .01 & .28 & .07 \\
\hline
\end{tabular}

Note: "-" indicates error free performance; $\mathrm{O} 1=$ participant 1 in the OR condition.

As shown in Figure B1, this is due to the partial collapse of the $\mathrm{HH}$ and HL functions. However, this violations does not appear to be too severe. We opted to retain participant O2 but remove $\mathrm{O} 5$ and A1 from the non-parametric analyses. We do continue to provide the data for these participants but do not discuss them. We do retain all participants for our parametric computational modeling analyses which is not dependent on the assumption of selective influence.

\section{Double Change Items}

Figures 5 and 6 show the SICs along with the $95 \%$ bootstrapped confidence intervals for the OR Task and AND Task, respectively. Additionally, the MIC is provided for each participant. For the OR Task, it can be seen that all SICs have a positive portion, which is greater than the negative portion. This is most consistent with a coactive or parallel selfterminating architecture. Although each SIC appears to have an initial negative deflection, this part of the curve always contains 0 within its confidence bounds. This suggests that the evidence for coactivity is not strong.

For the AND Task, the SICs for A2 and A3 appear to have positive and negative portions which are approximately equal, which is most indicative of serial exhaustive processing. For A4 and A5 the SIC is primarily negative, which is consistent with parallel exhaustive processing. 
Table 4

Observed Mean Correct and Error RTs (ms), and Error Rates for Individual Stimuli for each Participant in Experiment 1: AND Task

\begin{tabular}{llrrrrrrrrr}
\hline & & & \multicolumn{1}{c}{ Item } \\
\hline Participant & Variable & HH & HL & LH & LL & HS & LS & SH & SL & SS \\
\hline A1 & RT correct & 713 & 717 & 696 & 789 & 779 & 763 & 886 & 762 & 699 \\
& RT error & 784 & 765 & 780 & 756 & 811 & 803 & 709 & 785 & 844 \\
& p(error) & .07 & .28 & .08 & .51 & .57 & .22 & .94 & .42 & .13 \\
A2 & RT correct & 637 & 810 & 765 & 939 & 720 & 817 & 768 & 805 & 695 \\
& RT error & 896 & 996 & 838 & 956 & 869 & 1070 & 880 & 942 & 1165 \\
& p(error) & .01 & .05 & .20 & .24 & .03 & .08 & .12 & .12 & .01 \\
A3 & RT correct & 950 & 1119 & 1165 & 1328 & 1008 & 1056 & 908 & 928 & 817 \\
& RT error & 733 & 1218 & 1232 & 1380 & 1200 & 1336 & 1406 & 1241 & 915 \\
& p(error) & .004 & .05 & .09 & .18 & .08 & .03 & .12 & .09 & .01 \\
A4 & RT correct & 982 & 1301 & 1234 & 1447 & 1141 & 1138 & 1041 & 959 & 926 \\
& RT error & 1005 & 1411 & 1427 & 1415 & 1408 & 1772 & 1421 & 1409 & 1033 \\
& p(error) & .02 & .08 & .15 & .16 & .08 & .06 & .07 & .07 & 0.01 \\
A5 & RT correct & 1177 & 1461 & 1502 & 1710 & 1431 & 1485 & 1880 & 1805 & 1494 \\
& RT error & 1978 & 1868 & 1879 & 1841 & 1605 & 1954 & 1805 & 1861 & 2091 \\
& p(error) & .01 & .07 & .22 & .31 & .15 & .16 & .27 & .30 & 0.04 \\
\hline
\end{tabular}

Note: "-" indicates error free performance; A1 = participant 1 in the AND condition.

Table 5

Directional KS tests of the SIC for Experiment 1: OR task and AND task

\begin{tabular}{lcccc}
\hline Participant & $\mathrm{D}+$ & $\mathrm{p}$ & $\mathrm{D}-$ & $\mathrm{p}$ \\
\hline O1 & 0.20 & $.09^{*}$ & 0.11 & .45 \\
O2 & 0.25 & $.01^{*}$ & 0.05 & .86 \\
O3 & .42 & 0.10 & .52 \\
O4 & 0.12 & $.03^{*}$ & 0.11 & .46 \\
\hline Participant & $\mathrm{D}+$ & $\mathrm{p}$ & $\mathrm{D}-$ & $\mathrm{p}$ \\
\hline A2 & 0.11 & $.22^{*}$ & 0.29 & $<.001^{*}$ \\
A3 & 0.11 & $.26^{*}$ & 0.17 & $.03^{*}$ \\
A4 & 0.01 & .98 & 0.19 & $.01^{*}$ \\
A5 & 0.02 & .96 & 0.18 & $.03^{*}$ \\
\hline
\end{tabular}

Note: * indicates a significant difference with an alpha level of .33.

$\mathrm{D}+$ tests whether the SIC is significantly greater than zero.

D- tests whether the SIC is significantly lower than zero. 

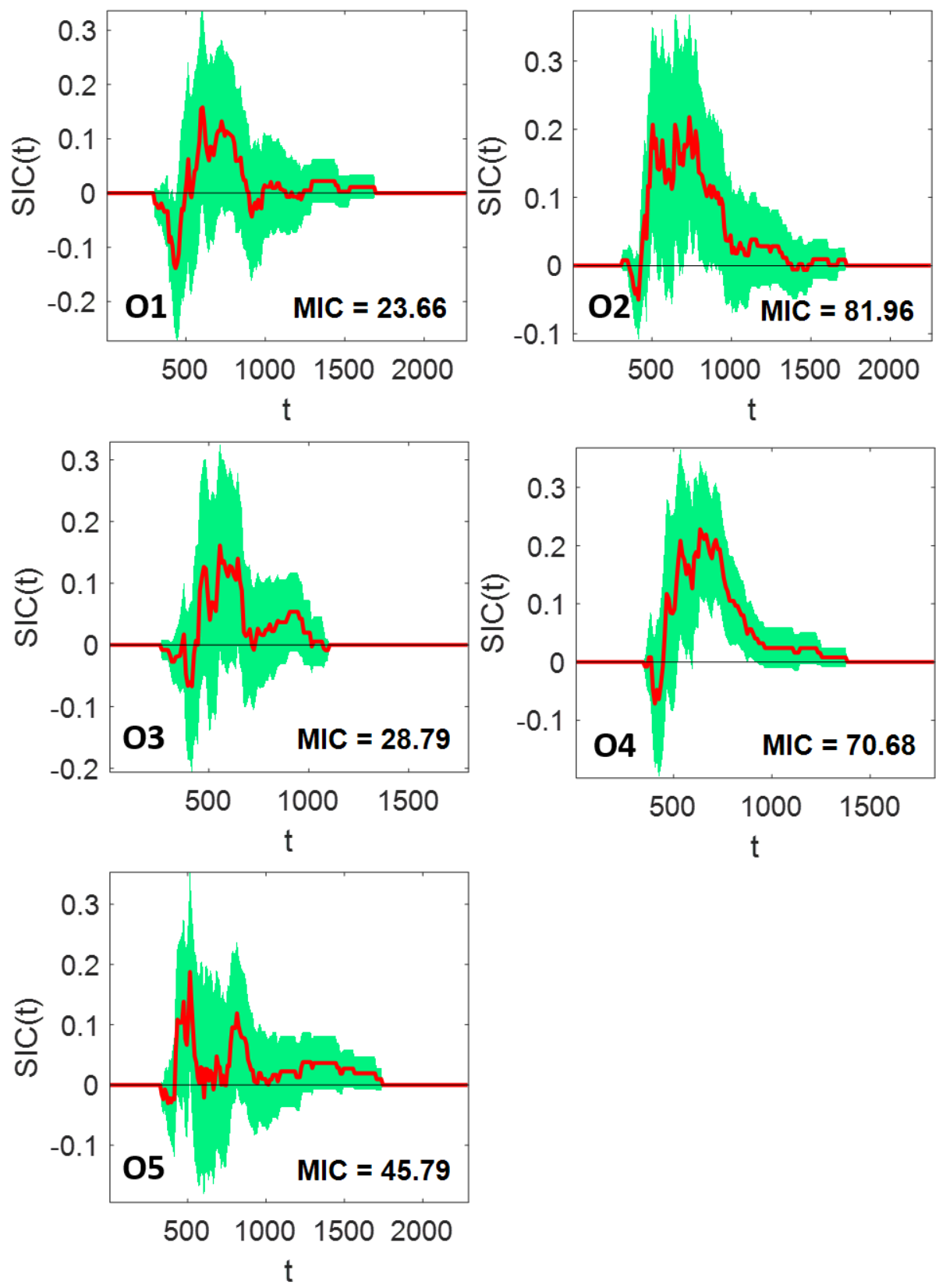

Figure 5. Observed double change item SICs (red line) for individual participants in Experiment 1: OR Task. Blue lines represent 95\% bootstrapped CIs. Note that participant O5 showed substantial violations of stochastic dominance. 

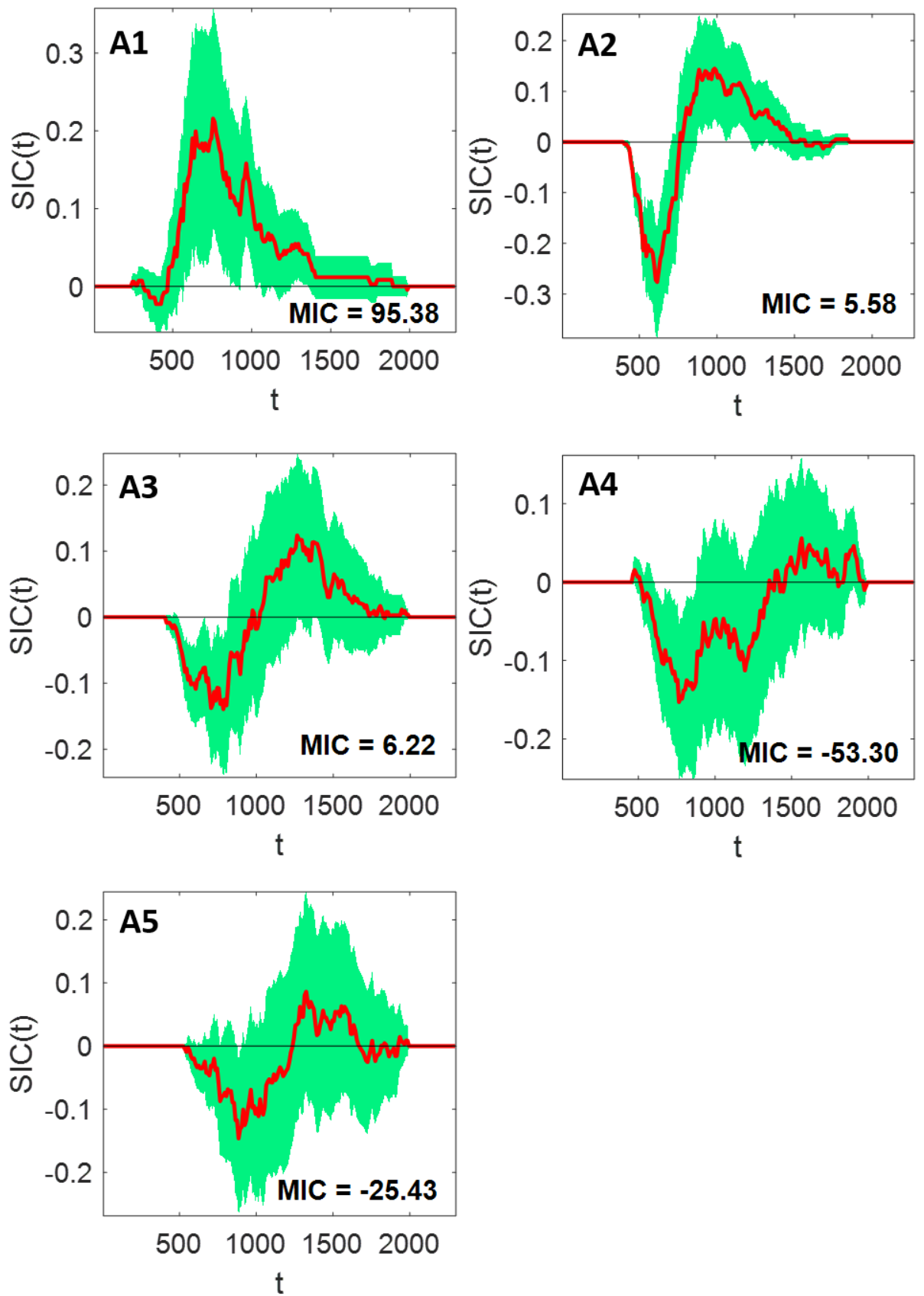

Figure 6. Observed double change item SICs (red line) for individual participants in Experiment 1: AND Task. Blue lines represent 95\% bootstrapped CIs. Note that participant A1 showed substantial violations of stochastic dominance. 
Table 6

Statistical results for Experiment 1: OR Task

\begin{tabular}{|c|c|c|c|c|c|c|}
\hline \multirow[b]{2}{*}{ Effect } & \multicolumn{3}{|c|}{$\mathrm{O} 1$} & \multicolumn{3}{|c|}{$\mathrm{O} 2$} \\
\hline & $d f$ & $F$ & $\mathrm{p}$ & $d f$ & $F$ & $\mathrm{p}$ \\
\hline Session & 3 & 17.16 & $<.001$ & 3 & 38.28 & $<.001$ \\
\hline Left & 1 & 12.28 & $<.001$ & 1 & 81.17 & $<.001$ \\
\hline Right & 1 & 22.83 & $<.001$ & 1 & 15.45 & $<.001$ \\
\hline Session $\mathrm{x}$ L & 3 & 2.01 & .11 & 3 & 1.85 & .14 \\
\hline Session $\mathrm{x} R$ & 3 & 0.37 & .77 & 3 & 0.91 & .44 \\
\hline Left x Right & 1 & 0.85 & .36 & 1 & 7.49 & .006 \\
\hline Session $\mathrm{x} L \mathrm{x} \mathrm{R}$ & 3 & 0.64 & .59 & 3 & 0.22 & .88 \\
\hline \multirow[t]{2}{*}{ Error } & 431 & & & 476 & & \\
\hline & \multicolumn{3}{|c|}{ O3 } & \multicolumn{3}{|c|}{$\mathrm{O} 4$} \\
\hline Effect & $d f$ & $F$ & $\mathrm{p}$ & $d f$ & $F$ & $\mathrm{p}$ \\
\hline Session & 3 & 11.42 & $<.001$ & 3 & 4.39 & .005 \\
\hline Left & 1 & 20.56 & $<.001$ & 1 & 22.99 & $<.001$ \\
\hline Right & 1 & 29.11 & $<.001$ & 1 & 44.41 & $<.001$ \\
\hline Session $x \mathrm{~L}$ & 3 & 1.68 & .17 & 3 & 1.03 & .38 \\
\hline Session $x \mathrm{R}$ & 3 & 2.53 & .06 & 3 & 0.37 & .78 \\
\hline Left $\mathrm{x}$ Right & 1 & 1.68 & .20 & 1 & 10.03 & .002 \\
\hline Session $\mathrm{x} L \mathrm{x} \mathrm{R}$ & 3 & 0.36 & .78 & 3 & 0.69 & .56 \\
\hline Error & 434 & & & 478 & & \\
\hline
\end{tabular}
$\mathrm{H}) \times 2$ (Right change: L vs H) ANOVAs for each individual participant (see Table 6). For the AND condition, as participants completed an additional session, we conducted a series of 5 (Sessions) $\times 2$ (Left change: $\mathrm{L}$ or $\mathrm{H}) \times 2$ (Right change: $\mathrm{L}$ or $\mathrm{H})$ ANOVAs for these participants (see Table 7).

In general, we find the following across all participants (exceptions noted):

1. There was a main effect of session for all participants except for A2, indicating that RTs become shorter over the course of the experiment.

2. There was a significant main effect of change salience for both discs indicating that the change discriminability manipulation was effective.

3. Session did not interact with either dimension, indicating the left and right channel changes tended to be processed at the same rate across sessions.

4. The three-way interaction was not significant, indicating a stable relationship between double change items across sessions. This indicates that participants were not changing processing strategy from session to session.

For the OR condition, we conducted a series of 4 (Session) $\times 2$ (Left change: L vs 
Table 7

Statistical results for Experiment 1: AND Task

\begin{tabular}{|c|c|c|c|c|c|c|}
\hline \multirow[b]{2}{*}{ Effect } & \multicolumn{3}{|c|}{$\mathrm{A} 2$} & \multicolumn{3}{|c|}{ A3 } \\
\hline & $d f$ & $F$ & $\mathrm{p}$ & $d f$ & $F$ & $\mathrm{p}$ \\
\hline Session & 4 & 2.03 & .09 & 4 & 29.07 & $<.001$ \\
\hline Left & 1 & 69.45 & $<.001$ & 1 & 71.23 & $<.001$ \\
\hline Right & 1 & 123.77 & $<.001$ & 1 & 68.27 & $<.001$ \\
\hline Session $\mathrm{x}$ L & 4 & 0.74 & .57 & 4 & 0.55 & .70 \\
\hline Session $\mathrm{x} R$ & 4 & 2.05 & .09 & 4 & 0.17 & .95 \\
\hline Left $x$ Right & 1 & 0.02 & .88 & 1 & 0.15 & .70 \\
\hline Session $\mathrm{x} L \mathrm{x} R$ & 3 & 0.50 & .73 & 4 & 1.37 & .24 \\
\hline \multirow[t]{2}{*}{ Error } & 819 & & & 832 & & \\
\hline & \multicolumn{3}{|c|}{$\mathrm{A} 4$} & \multicolumn{3}{|c|}{ A5 } \\
\hline Effect & $d f$ & $F$ & $\mathrm{p}$ & $d f$ & $F$ & $\mathrm{p}$ \\
\hline Session & 4 & 22.24 & $<.001$ & 4 & 10.47 & $<.001$ \\
\hline Left & 1 & 52.49 & $<.001$ & 1 & 33.82 & $<.001$ \\
\hline Right & 1 & 122.13 & $<.001$ & 1 & 43.92 & $<.001$ \\
\hline Session x L & 4 & 0.82 & .17 & 4 & 0.50 & .74 \\
\hline Session $x \mathrm{R}$ & 4 & 1.56 & .06 & 4 & 0.93 & .46 \\
\hline Left x Right & 1 & 1.26 & .20 & 1 & 0.00 & .99 \\
\hline Session $\mathrm{x} L \mathrm{x} R$ & 4 & 0.07 & .78 & 4 & 1.60 & .17 \\
\hline Error & 781 & & & 609 & & \\
\hline
\end{tabular}

In the OR Task, the interaction (see Figure 5) was significant for $\mathrm{O} 2$ and $\mathrm{O} 4$ which provides support for either a parallel self-terminating, coactive-min, or MAX model account of processing strategy. However, for $\mathrm{O} 1$ and $\mathrm{O} 3$ this interaction was not significant. Although the MIC was positive for these participants, the non-significant interaction is consistent with serial processing. Nonetheless, caution must be taken when interpreting a null result in this context since the MIC with a p-value criterion of .05 is biased toward the serial model. In the AND Task, the Left Change $\times$ Right Change interaction (see Figure 6) was not significant for any of the participants, consistent with the serial model and MAX-first model accounts.

Turning to the shape of the SIC, for the OR condition the SIC is largely positive (see Figure 5). For most participants, with the exception of O1, there does not appear to be a significant early negative deflection. For the AND task, all participants have an S-shaped SIC with roughly equivalent negative and positive portions (see Figure 6). One exception is participant A4 who appears to have a larger negative than positive portion.

Both positive and negative D-tests are displayed in Table 5 for the OR and AND tasks. For three of participants in the OR Task, the positive deflection was significantly different to zero whereas the negative deflection was not. This is consistent with parallel self-terminating processing, coactive-min, or the MAX model. For O3, however, neither positive nor negative deflections were significantly different from zero, consistent with serial 


\footnotetext{
${ }^{4}$ Unlike the OR task, the single change trials in the AND task offer us additional non-parametric diagnostic information (Fifić et al., 2010; see also Little et al., 2015, 2018). For completeness, we report these analyses in the supplementary materials.
}

self-terminating processing. For the AND Task, A2 and A3 both had positive and negative portions which were significantly different to zero, consistent with serial processing. A4 and A5, however, only had negative portions which were significantly different from zero, indicative of parallel exhaustive processing.

\section{OR Task Single Change Trials}

The single change trials are not diagnostic of processing architecture for the OR task using SFT; however, there are still aspects of the data worth considering. A summary of the mean RTs and accuracy for the single change trials, alongside the double change trials, for each participant is presented in Table 3 . We included participant O5 in this analysis.

For all participants, the high salience single targets were processed faster and more accurately than the low salience single targets. All of these differences were significant. The smallest RT benefit was for participant O2 (High minus Low $=-256$ msec; $t(375)=$ $-9.20, p<.001$ ), and the smallest accuracy benefit was for participant O4 (High minus Low $=.23, t(374)=7.45, p<.001)$.

We additionally compared whether the left targets were detected faster or more accurately than the right targets. All participants were significantly less accurate for the left target than the right target, with $\mathrm{O} 2$ having the largest difference (Left minus Right $=.20$, $t(372)=-4.34, p<.001)$. However, only participant O2 showed a significant difference in response time, with the left location detected faster than the right (Left minus Right $=94$ msec, $t(375)=-3.09, p<.001)$.

For all participants, trials with two changes were faster and more accurate than trials with one change (i.e., a redundant target effect). All of these differences were significant. The smallest RT benefit was for participant O2 (Double minus Single Target $=-28 \mathrm{msec}$; $t(877)=-2.89, p<.001$ ), and the smallest accuracy benefit was for participant O4 (Double minus Single Targets $=.11, t(877)=7.81, p<.001)$.

\section{AND Task Single Change Trials}

For the AND task, we focused again on comparing the salience of the targets, the location of the targets, and the redundant target effect ${ }^{4}$. We included participant A1 in this analysis.

The high salience single targets were not systematically faster or more accurate than the low salience targets. With the exception of A2, none of the participants showed a significant difference in mean RT or accuracy (largest t-value $=1.55, p=.12$ ). For A2, the high salience single targets were faster than the low salience single (High minus low $=77$ msec, $t(718)=-4.19, p<.001)$.

The was a tendency for the left location to be different from the right, but this was not systematic. Participants A1, A3, and A4 were slower on the left than the right (largest Left minus Right $=132 \mathrm{msec}, t(678)=4.79, p<.001)$. A5 was faster on the left than the right (Left minus Right $=-169 \mathrm{msec} ; t(449)=-4.90, p<.001$ ). All of the participants, 
save A4, were more accurate on the left than the right (largest Left minus Right $=.29$, $t(710)=8.08, p<.001)$.

Two participants (A1 and A5) had significant redundant target benefits for both RT (largest Double minus Single RT $=-95$ msec, $t(1154)=-4.34, p<.001$ ) and accuracy (largest Double minus Single accuracy $=.3, t(1665)=13.34, p<.00)$. By contrast, participant A3 and A4 were slower in responding to the double targets compared to the single targets (largest Double vs Single target difference $=135 \mathrm{msec}, t(1622)=7.20, p<.001$ ) . $\mathrm{A} 2$ and A4 were also less accurate on the redundant target (largest Double vs Single target difference $=-.04, t(1567)=-2.65, p<.001)$.

\section{Capacity Analysis}

A number of different mechanisms can produce a redundant target effect including probability summation (i.e., minimum time processing of a parallel race model, Raab, 1962; Townsend \& Ashby, 1983) and facilitatory interactions between channels (Eidels et al., 2011; Mordkoff \& Miller, 1993; Mordkoff \& Yantis, 1991); consequently, to compare the efficiency of double change detections to single change detections, we employ a measure which characterises the extent to which performance deviates, positively or negatively, from expectations of a baseline parallel process (Townsend \& Nozawa, 1995). To compute capacity, we pooled the response times from correct trials across all double target items and the response times from correct trials from all single target items (into either left or right changes) and applied Equation 6 for the OR task and Equation 8 for the AND task.

Before presenting these results, we note an important caveat to their interpretation. First, as illustrated by Mordkoff and Yantis (1991) and Mordkoff and Miller (1993), the presentation frequencies of each of the relevant trial types can lead to contingencies and response biases which can lead to violation of the Race Model Inequality (Miller, 1982). Capacity can be thought of as a generalization of the measure of the Race Model Inequality (Townsend \& Eidels, 2011; Townsend \& Nozawa, 1995) and is therefore also affected by these contingencies (Houpt et al., 2014). In a nutshell, contingencies that favor redundant target processing can lead to faster than the expected minimum time processing (e.g., in the OR case) pushing capacity to be greater than 1 . These contingencies cannot be fully eliminated in the double factorial design (Houpt et al., 2014) and are present here. Since the analysis of capacity is our main concern in Experiment 2, we defer discussion of the effect of these contingencies, and simply report the capacity results here. The capacity results for the OR condition are shown in Figure 7 . In all cases, the capacity coefficient is less than 1 but within the lower Grice bound.

The capacity results for the AND condition are shown in Figure 8. For most participants, capacity starts high (in some cases above the upper bound) and then decreases over time. The exception is A5 whose capacity curve is greater than the upper bound for all $t$.

\section{Discussion}

For most observers, the OR task SIC was overadditive indicative of either parallel self-termination, coactive-min or MAX model processing. The SICs for the OR task do contain a slight "negative-blip" (cf. Townsend \& Nozawa, 1995) for shorter RTs which could indicate coactive-max processing. However, the $95 \%$ CIs incorporate zero and the D- tests 

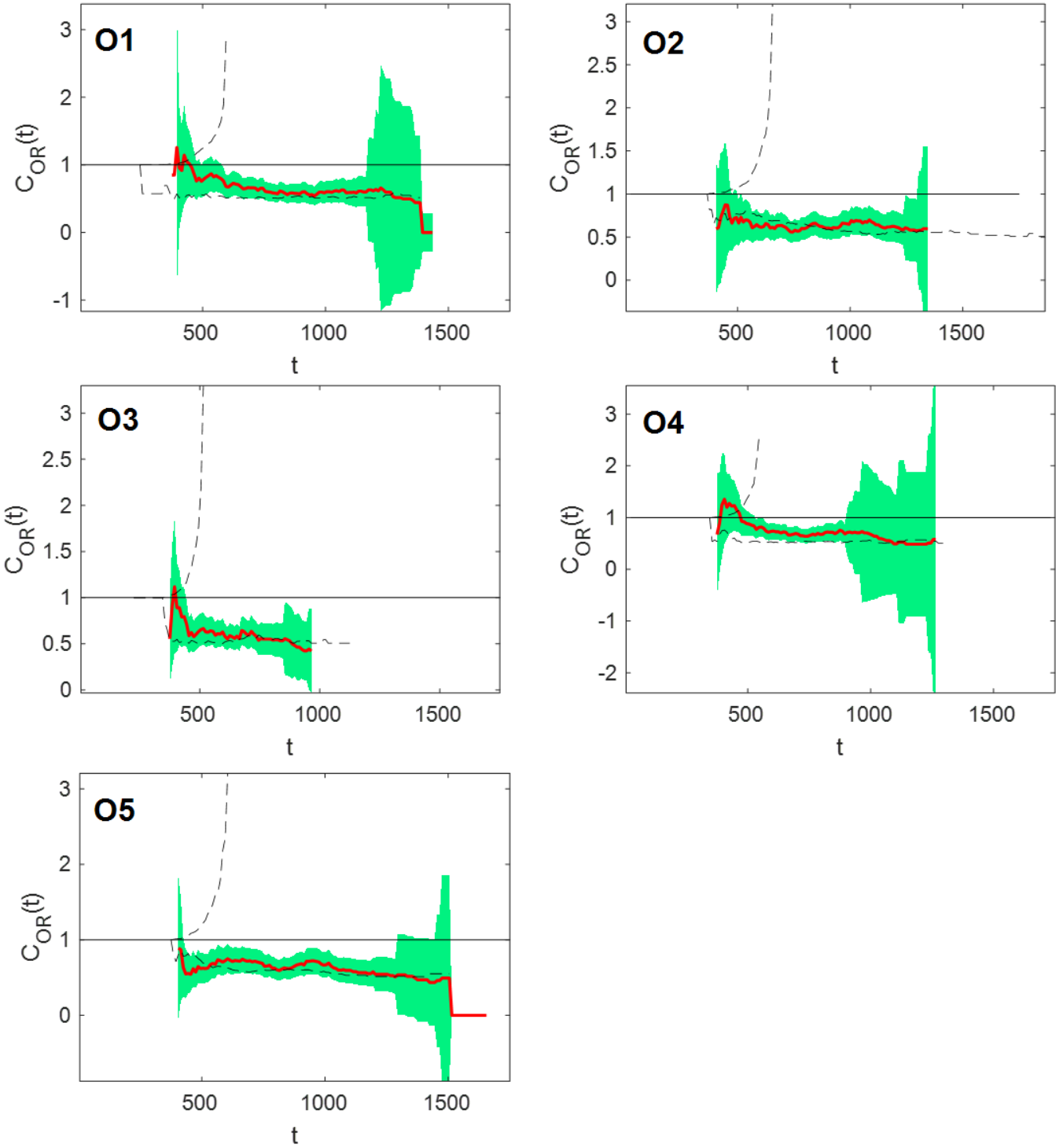

Figure 7. Capacity results for the OR condition from Experiment 1. $C_{O R}(t)$ along with the $95 \%$ bootstrapped confidence intervals are shown. The upper dotted line is the Race Model Inequality bound (Miller, 1982), and the bottom dotted line is the Grice bound (Grice et al., 1984). The bounds provide the upper and lower limits on the predictions of the baseline unlimited capacity parallel model. 

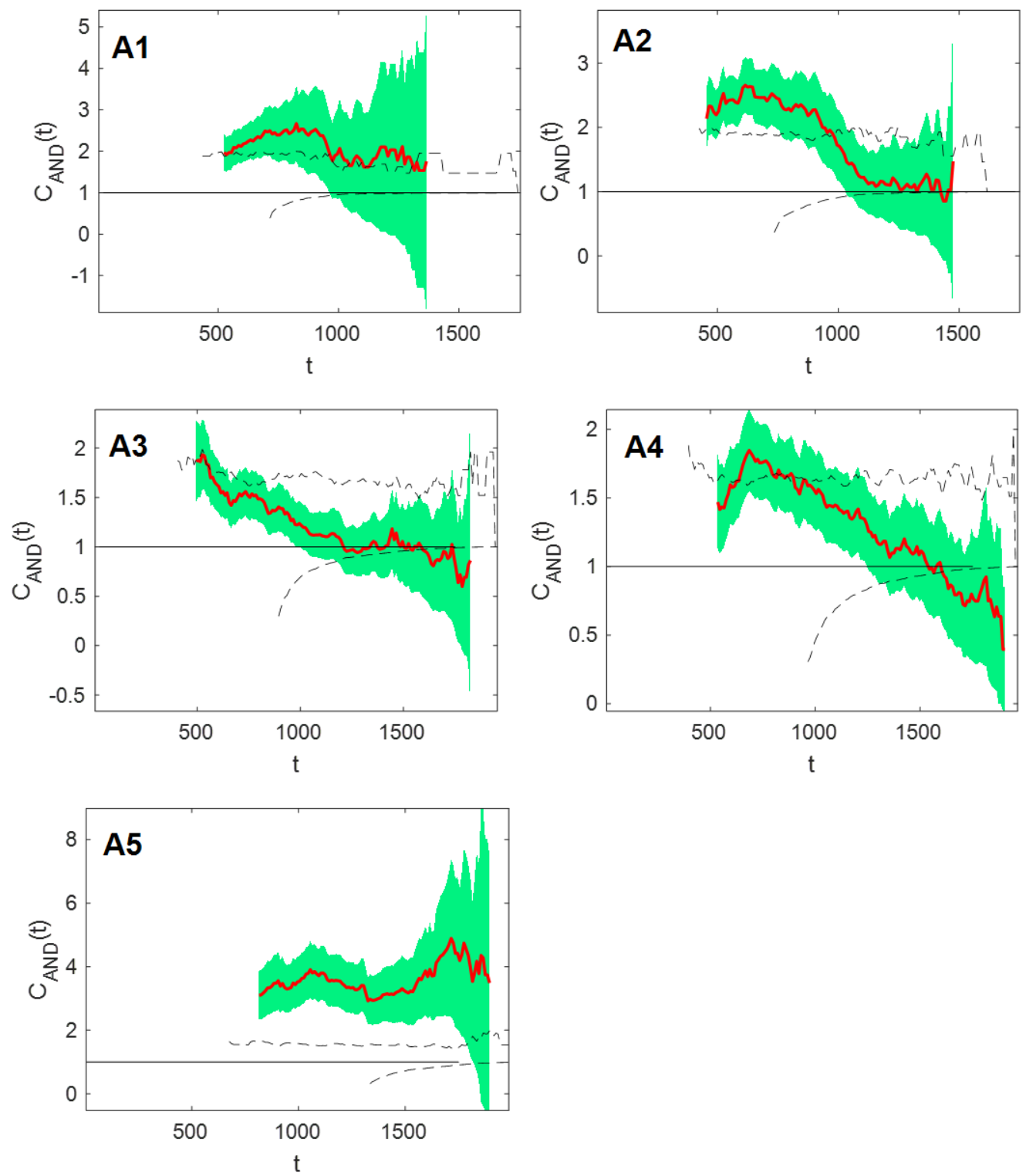

Figure 8. Capacity results for the AND condition from Experiment 1. $C_{A N D}(t)$ along with the $95 \%$ bootstrapped confidence intervals are shown. The upper and lower dotted lines are the Colonius-Vorberg bounds (Colonius \& Vorberg, 1994). The bounds provide the upper and lower limits on the predictions of the baseline unlimited capacity parallel model. 
were not significant, even at a less-conservative level, so it is unclear the extent to which coactivity can predict observed OR results. The AND task SIC, on the other hand, was additive for most observers, consistent with serial exhaustive processing (for the double targets) or a MAX-first model. The non-parametric analyses allow us to rule out some processing accounts, but there remain several candidates which might explain processing. To provide further insight, we instantiated these accounts using our folded-Normal-LBA models and compared the quantitative model fits between different accounts.

\section{Computational Model Fitting}

Our focus thus far has been on the non-parametric analysis of performance on the double change trials. However, by implementing the relevant models parametrically, we complement and extend our non-parametric analyses. First, in the analysis of the SIC, the single change items are not used. Computational modeling allows us to consider both single and double change items in both OR and AND designs simultaneously. Second, the nonparametric analyses only utilize correct responses, whereas with computational modeling we are able to fit both correct and error RT distributions, affording a more comprehensive understanding of the data. Third, we are able to compare the different quantitative results of models which make identical SIC predictions. Fourth, we can explore more flexible representations and determine whether they improve the fit of the model relative to their complexity.

With regard to this last item, we fit two versions of each model. We term the first version fixed variance. While this version assumes that the perception of a change on the left may be different from a change on the right (capturing difference in sensitivity to luminance increments and decrements), the fixed variance models assume that the variability in the perception of the low salience change is the same as the variability of a high salience change, and, for that matter, the same variability as perceiving no change. This is similar in spirit to an equal variance signal detection model. We suspect that this simplifying assumption is incorrect; however, the extent to which it matters in comparing across different models is unknown. We consequently also fit a free variance model, which allowed the perceptual variability to differ between no change, low change, and high change.

Summary of Models. Drift rate. A schematic summary of the models is shown in Figure 1 and 2. For each model, the drift rates are derived from the folded-normal representation of change strengths. For the coactive-min and coactive-max models, we use the bivariate folded normal representation and integrate the region associated with either the minimum or maximum evidence, respectively (see Figure 2). For the remaining models, the mean drift rate at each location is derived by integrating the marginal folded-normal distributions. For the MAX model, only the maximum of the mean drift rates is used to drive a single accumulation process. Hence, each model has parameters for the standard deviation at the left and right locations $\left(\sigma_{x}\right.$ and $\left.\sigma_{y}\right)$ as well as parameters for the decision bounds for the left and right locations $\left(d b_{x}\right.$ and $\left.d b_{y}\right)$. The bivariate distribution of the coactive model allows for a more complicated covariance structure. For simplicity and since we were interested in the comparison across different architecture, we set the covariance equal to 0 . The mean of the folded-normal distribution was set to 0 for no-change, 1 for a low change, and 2 for a high change. 


\footnotetext{
${ }^{5}$ We also fit "rule-breaking" versions of the parallel and serial models which always self-terminate as soon as the faster of the two locations makes a decision (Bushmakin et al., 2015). These models did not fit as

well as any of the other models, and we so omit further discussion of them.
} (Brown \& Heathcote, 2008). The LBA represents a choice between $N$ alternatives using $N$ accumulators (one for each response: change vs no-change). In the LBA, each trial begins with a starting amount of evidence $(k)$ drawn from a Uniform distribution bounded at $(0$, A) that increases linearly at an amount given by the drift rate $(v)$, which is drawn from a normal distribution with a mean, $\nu_{\text {change }}$ or $1-\nu_{\text {change }}$ for the Change and no-change accumulators, respectively, and a standard deviation of $s$. Evidence accumulates until a response threshold $(b)$ is reached. The first accumulator to reach a decision provides the decision time. The overall response time is the decision time plus a non-decision time $\left(t_{0}\right)$ which is a constant parameter.

Processing Architectures. In the coactive-min, coactive-max, and MAX models, there is a single pair of accumulators which race to determine the response and decision time. Hence, there are parameters for $s, A, b_{\text {Change }}, b_{\text {NoChange }}$, and $t 0$ in these models. These models differ in how the drift rate is calculated, either by integrating different regions of the decision space or by taking the maximum mean rate at each location.

The parallel, serial, and MAX-first models assume that decisions about changes in each location occur independently and can be modeled using independent evidence accumulation processes for each dimension. Hence, there are two pairs of LBA accumulators, one for each location. The minimum and maximum integration rules then conceptually map on to the self-terminating and exhaustive stopping rules, respectively (see Figure 1).

These models differ in how the information from each pair of accumulators is combined to determine the overall decision time. For the parallel exhaustive model, the overall decision time is the maximum decision time generated by each pair of accumulators regardless of whether the Change or No Change accumulator finishes first in each pair. For the parallel self-terminating model, we assume that the model terminates as soon as it is logically possible to do so. The overall decision time is therefore the minimum decision time generated by each pair of accumulators when both of the Change accumulator finishes faster in both pairs. If only one Change accumulator finishes faster, then the decision time is determined only by that pair. Conversely, if both of the No Change accumulators finish faster, the overall decision time is the maximum time. This captures the idea that processing of the SS item condition is logically exhaustive, even in the OR case, but that processing will terminate as soon as a change is detected ${ }^{5}$.

For the serial model, we applied a similar set of logical constraints. Serial exhaustive processing involved summing together the decision times of each pair of accumulators. Serial self-terminating processing was more complex in that whether or not the model terminates processing after detecting a change depends on which location is processed first. If a change is processed first, then the serial self-terminating model stops at that point, and the decision time is determined by only that accumulation process. If no-change is detected at the first processed location, then the serial self-terminating model continues to the second location, and the overall decision time is the sum of the decision times at each location. Hence, for the serial self-terminating model, we estimated an additional parameter, $p_{\text {Left }}$, capturing the probability that the left location was processed first. The MAX-first model is a special

Evidence Accumulation. The accumulation of evidence is modelled using the LBA 
case of the serial self-terminating model in which the location with the maximum mean drift rate is always processed first; the MAX-first model proceeds to the other location only when no change is detected at the first-processed location.

For simplicity, we assumed that $A, b_{\text {Change }}, b_{N o C h a n g e}$, and $s$ were the same across pairs of accumulators (e.g., any location differences are captured by the drift rate and not by changes is response confidence). All of the models, therefore had 9 parameters, except for the serial self-terminating model, which had one additional parameter.

Free variance models. For the fixed variance versions of the models, the variance the folded-normal distribution was equivalent across each level of change strength. Although the $\sigma_{x}$ and $\sigma_{y}$ do allow for different representations at each location, because we fixed the mean location of the folded-normal distribution, there is a strong constraint on the perception of low and high changes. The free variance versions of the models each have four additional parameters: $\sigma^{\text {same }}, \sigma^{\text {low }}$, and $\sigma^{\text {high }}$ at both locations, $x$ and $y$.

Formal model fitting procedure. We used probability density approximation (PDA; Turner \& Sederberg, 2014) to find the likelihood for each model by simulating 50,000 data points from the model and using Holmes' method (2015). The log likelihood for each trial was then summed over items. Each participant was fit separately. For each parameter, we first transformed the parameter to lie on the whole real line and then adopted reasonably informative priors based on our prior work with these models (see e.g., Blunden et al., 2020; Cheng et al., 2018. The transformations and priors are shown in Table 8.

Table 8

Prior parameter distributions and transformations for each parameter

\begin{tabular}{lllll}
\hline Parameter & Transformation & Distribution & Prior Parameter & Values \\
\hline$d b_{x}$ & $\hat{d}_{x}=\operatorname{logit}\left[d b_{x}-x_{0}\right]$ & Normal & $\mu=0$ & $\sigma=.5$ \\
$d b_{y}$ & $\hat{d b}_{y}=\operatorname{logit}\left[d b_{y}-y_{0}\right]$ & Normal & $\mu=0$ & $\sigma=.5$ \\
$\sigma_{x}$ & $\hat{\sigma}_{x}=\log \left(\sigma_{x}\right)$ & Normal & $\mu=-1.5$ & $\sigma=.2$ \\
$\sigma_{y}$ & $\hat{\sigma}_{y}=\log \left(\sigma_{y}\right)$ & Normal & $\mu=-1.5$ & $\sigma=.2$ \\
$A$ & $\hat{A}=\log (A)$ & Normal & $\mu=-1.05$ & $\sigma=.2$ \\
$b_{\text {Change }}$ & $\hat{b}_{\text {Change }}=\log \left(b_{\text {Change }}-A\right)$ & Normal & $\mu=-1.05$ & $\sigma=1$ \\
$b_{N o-C h a n g e}$ & $\hat{b}_{\text {No-Change }}=\log \left(b_{N o-C h a n g e}-A\right)$ & Normal & $\mu=-1.05$ & $\sigma=1$ \\
$s$ & $\hat{s}=\log (s)$ & Normal & $\mu=-1.39$ & $\sigma=.5$ \\
$t_{0}$ & $\hat{t}_{0}=\log \left(t_{0}\right)$ & Normal & $\mu=-1.51$ & $\sigma=.2$ \\
$p_{x}$ & $\hat{p}_{x}=\operatorname{logit}\left(p_{x}\right)$ & Normal & $\mu=0$ & $\sigma=2$ \\
\hline
\end{tabular}

We used Differential Evolution Markov Chain Monte Carlo (DEMCMC; Turner, Sederberg, Brown, \& Steyvers, 2013) to efficiently generate proposals from the posterior distributions of each parameter. However, the variability in the likelihood approximation can cause the chains to become stuck if an accepted parameter set results in an usually high likelihood. To prevent the chains getting stuck in this manner, we re-sampled the likelihood of any existing chains each time the current proposal was rejected (Holmes, 2015). This led to a good mixing of the chains and strong convergence after the burn-in period.

For the fixed variance models, we used a burn-in period of 2150 iterations and for the free variance models we used a burn-in period of 3150 iterations. Between iterations 501-700 there was a deterministic migration step (Turner et al., 2013) every 20 iterations. 
The remaining sampling used a probabilistic migration step instead of a crossover step with a probability of .05 . The number of chains was determined by taking three times the number of parameters for each model. A minimum of 20000 posterior samples were taken per parameter, with 750 iterations estimated for each chain.

Model Comparison. For model comparison we used the Deviance Information Criterion (DIC; Gelman, Hwang, \& Vehtari, 2014). The deviance of a posterior sample of parameters, denoted $\theta$, is calculated as:

$$
D(\theta)=-2 \ln L(y \mid \theta)
$$

The DIC is calculated as:

$$
D I C=\bar{D}(\theta)+2 p_{D}
$$

Here, $\bar{D}(\theta)$ is the mean of the distribution of posterior deviances and $p_{D}=2 \operatorname{var}[\ln L(y \mid \theta)]$. The DIC punishes model complexity by penalizing the average negative log likelihood by a term which accounts for the functional form complexity of the model. The DIC can be thought of as a Bayesian version of a maximum likelihood-based fit statistic such as the Akaike Information Criteria (AIC; Akaike, 1974).

To summarise, we fit the coactive-min, coactive-max, serial, parallel, MAX, and MAXfirst models (see Table 1). These models instantiate both first-order (i.e., pooled) and second-order (i.e., independent) integration rules via coactivity (pooled) architectures and serial, parallel, and MAX (independent) architectures.

Because the computational models do not rely on the assumption of stochastic dominance, we also fit each of the models to participants $\mathrm{O} 5$ and $\mathrm{A} 1$, who were omitted in our previous analyses of the SIC.

The DICs for each individual participant in the OR fixed variance, OR free variance, AND fixed variance, and AND free variance are displayed in Tables 9 and 10, respectively. The preferred model is the model with the lowest DIC.

In the OR task, the majority of participants were best fit by a parallel self-terminating model (with one participant being best fit by the coactive-min model). For two participants (O2 and O5) the more flexible free variance model was preferred. For the remaining three participants (O1, O3, and O4) the DIC favoured the simpler fixed variance account.

For the AND task, the majority of participants were best fit by a serial self-terminating model, although two of the five participants were best fit by the coactive-max model. For the majority of participants (A1, A3, A4, and A5) the simpler, fixed variance models were preferred. For A2, the free variance serial self-terminating model provided the best fit to the data.

Along with model comparison estimates, it is essential to provide an estimate of how well a model fits the data to ensure that the model is capturing the data well (Heathcote, Brown, \& Wagenmakers, 2015). In the interest of space, we present the posterior predictions of the best fitting model for each item, for one participant, along with posterior parameter estimates of the parameters for the best fitting fixed variance model, for all participants. The posterior parameter estimates for the best fitting free variance models and posterior predictive estimates for the other participants are shown in the supplementary material. 


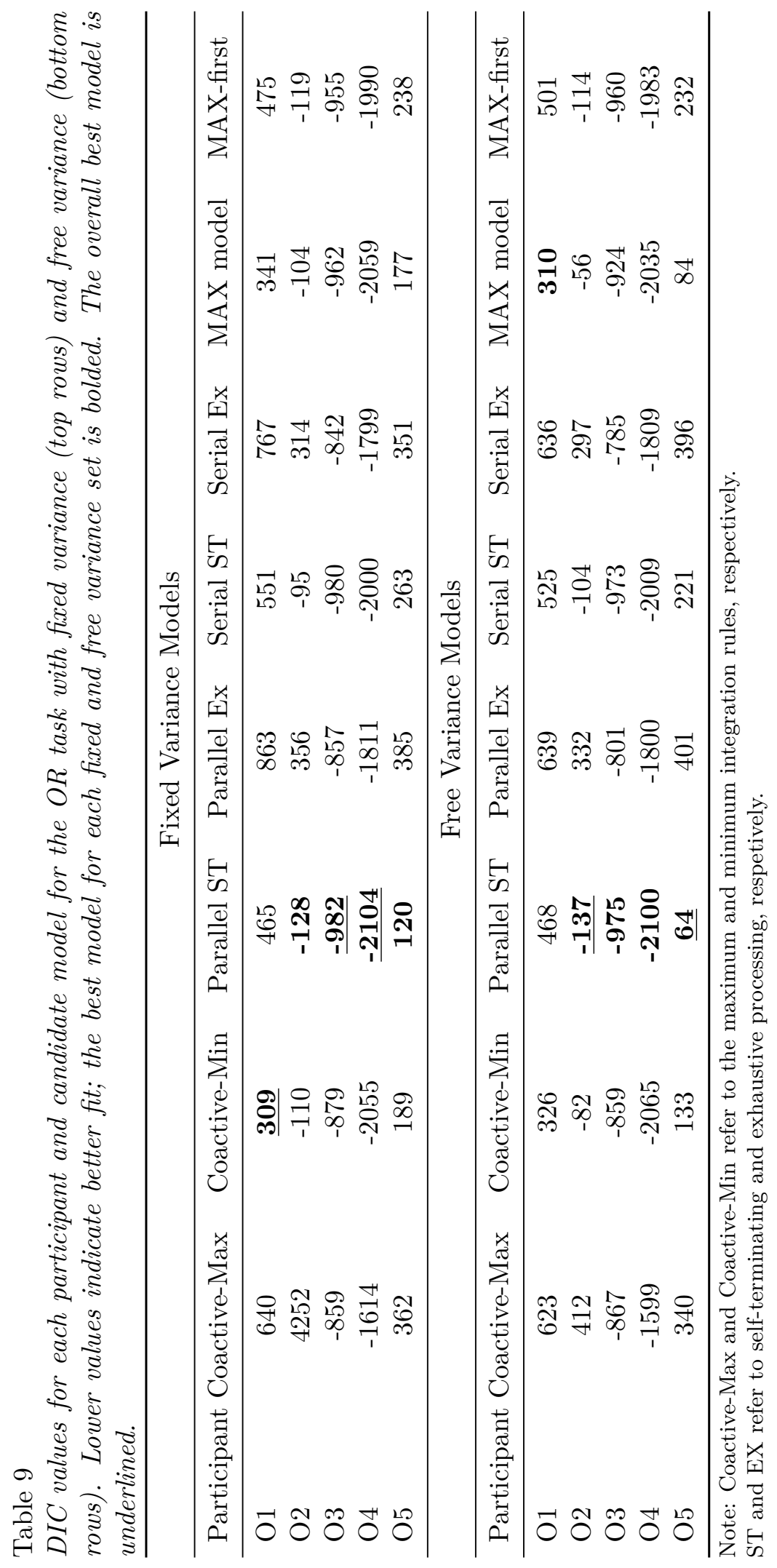




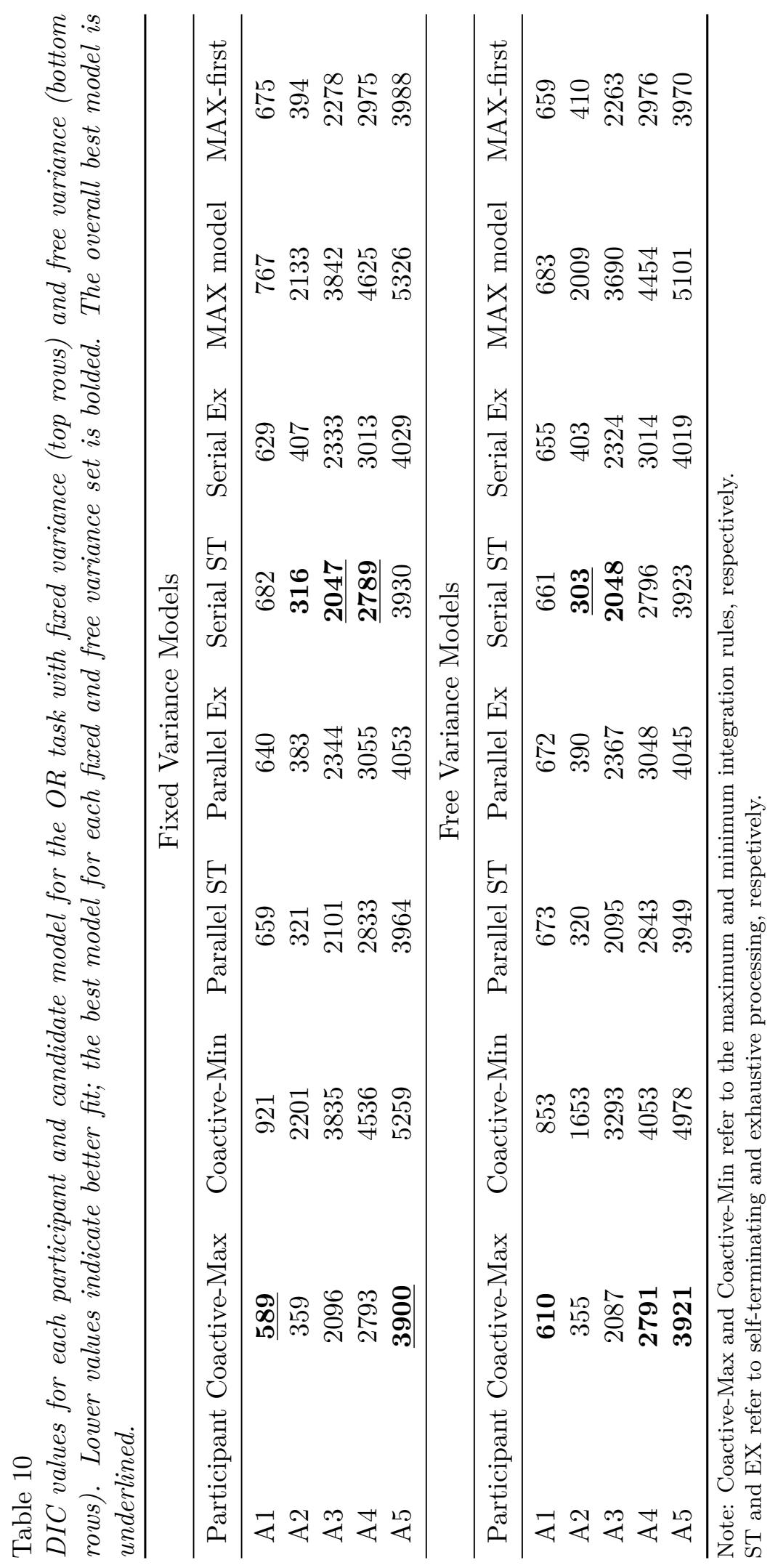


In Figure 9, we show the model predictions for O3 for the OR rule, and in Figure 10, we show the model predictions for A3 for the AND rule to show that the model provides a good fit to the data. The supplementary material shows that the fits of the best fitting models to the other participants are comparable. The posterior parameter estimates for each participant for the overall best fitting models for the AND and OR tasks are shown in Figures 11 and 12. It is clear from these figures that the models capture the proportion of responses along with the correct and error RTs accurately at the distributional level for each item.

Aside from answering our primary question of how changes from multiple locations are integrated, the posterior parameter values provide an explanation for some observations that we make of the data. For the OR condition, all of the participants have elevated error rates for low salience changes; the location of the decision bounds indicates some conservatism in responding change. That is, the boundaries for all participants are above .5 and closer to the low change region than the no change region at both the left and right locations. Consequently, all participants have at least some tendency to miss weak changes. Participant O4, who had the highest accuracy overall, had much higher perceptual variability for changes on the left and the right compared to the other participants along with lower values of the response criteria for change and no change responses. Finally, participant $\mathrm{O} 2$, who had the longest RTs overall, had higher response criteria than the other participants.

For the AND condition, all of the participants have good accuracy overall with the exception of A1 who performed poorly for most items. A1's posterior parameters suggest a quite different starategy from the other participants. The $p(X)$ parameter is shifted toward 1 indicating that the left location was almost exclusively processed before the right location. Likewise, the decision bound for the left location, $d b_{x}$, is very liberal indicating a propensity to report changes even when no change occurred. Coupled with the high uncertainty in the perceptual variability for the left location, $\sigma_{x}$, the model captures the high error rates for, e.g., the SH item (see Table 4).

In summary, the parameters governing of the change signal detection front-end component of the model used to generate the drift rate for each model contribute primarily to an explanation of the accuracy for each participant. The LBA parameters contribute primarily to the overall RT for each participant. By contrast, the different architectures explain the qualitative difference in the pattern of RTs across the double change trials as indicated by the MICs and SICs. As shown in the supplemental materials, although there was a tendency for the variability of the high salience changes to be less than the variability of the low salience changes, the $95 \%$ HDI's of the posterior difference had clear overlap with 0 . Likewise, there was no indication that the variability low salience changes were different from the perceived variability when there was no change. Consequently, although the free variance models fit better than the fixed variance models for a minority of subjects, this difference did not appear more generally or to a strong degree.

\section{Discussion}

Across both tasks, the qualitative SICs from the double target trials resemble the MAX and MAX-first predictions; however, it is apparent that explaining change detection required the influence of the weaker change location. For the OR task, the best fitting 

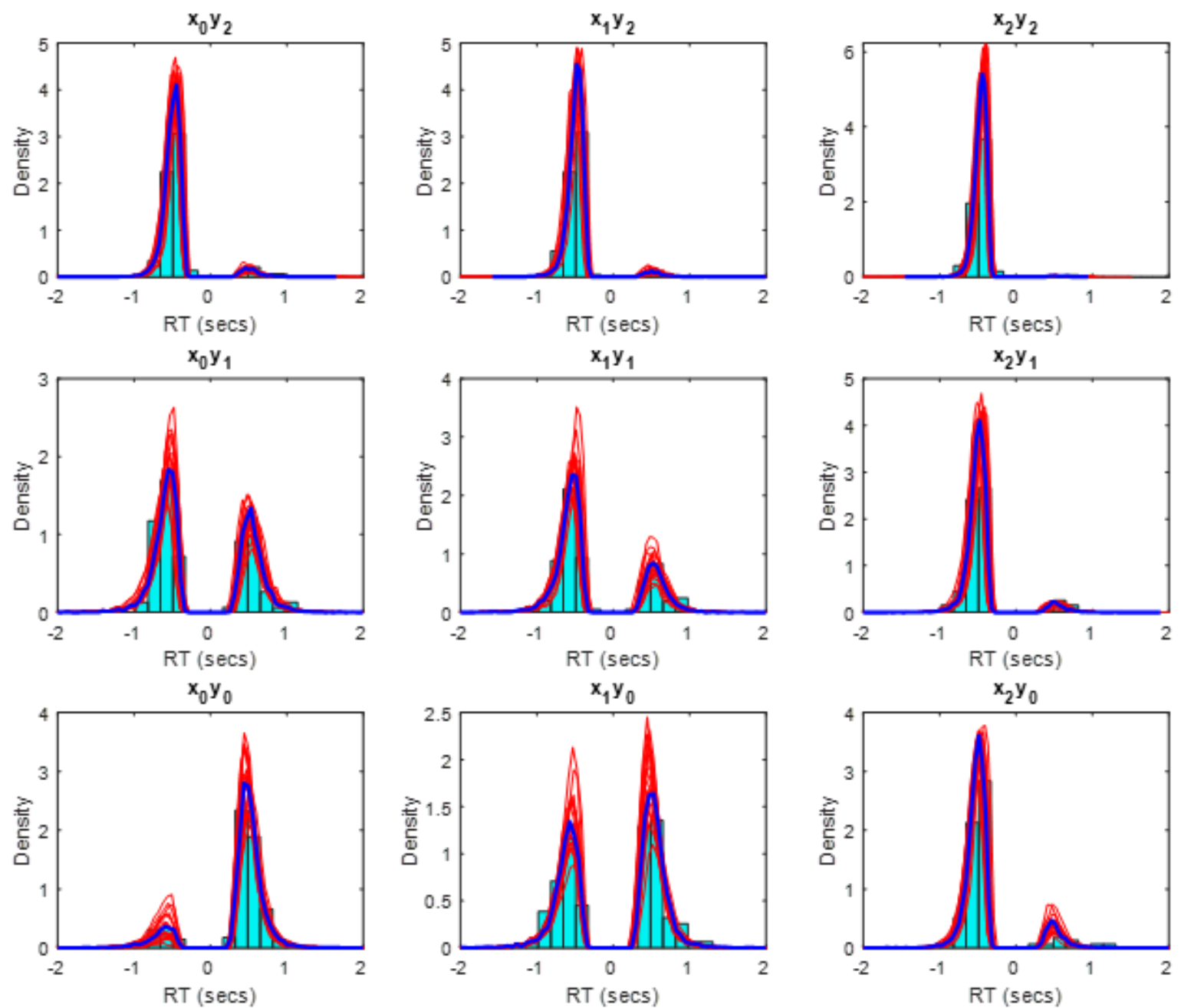

Figure 9. Posterior predictions from the fixed-variance parallel self-terminating model for observer O3. Each subplot shows the data and predictions for one item. Positive RTs indicate no change responses. Negative RTs indicate change responses. RT data are plotted in each histogram. Each red line indicates a posterior sample. The solid blue line indicates the predictions using the average posterior parameter values. 

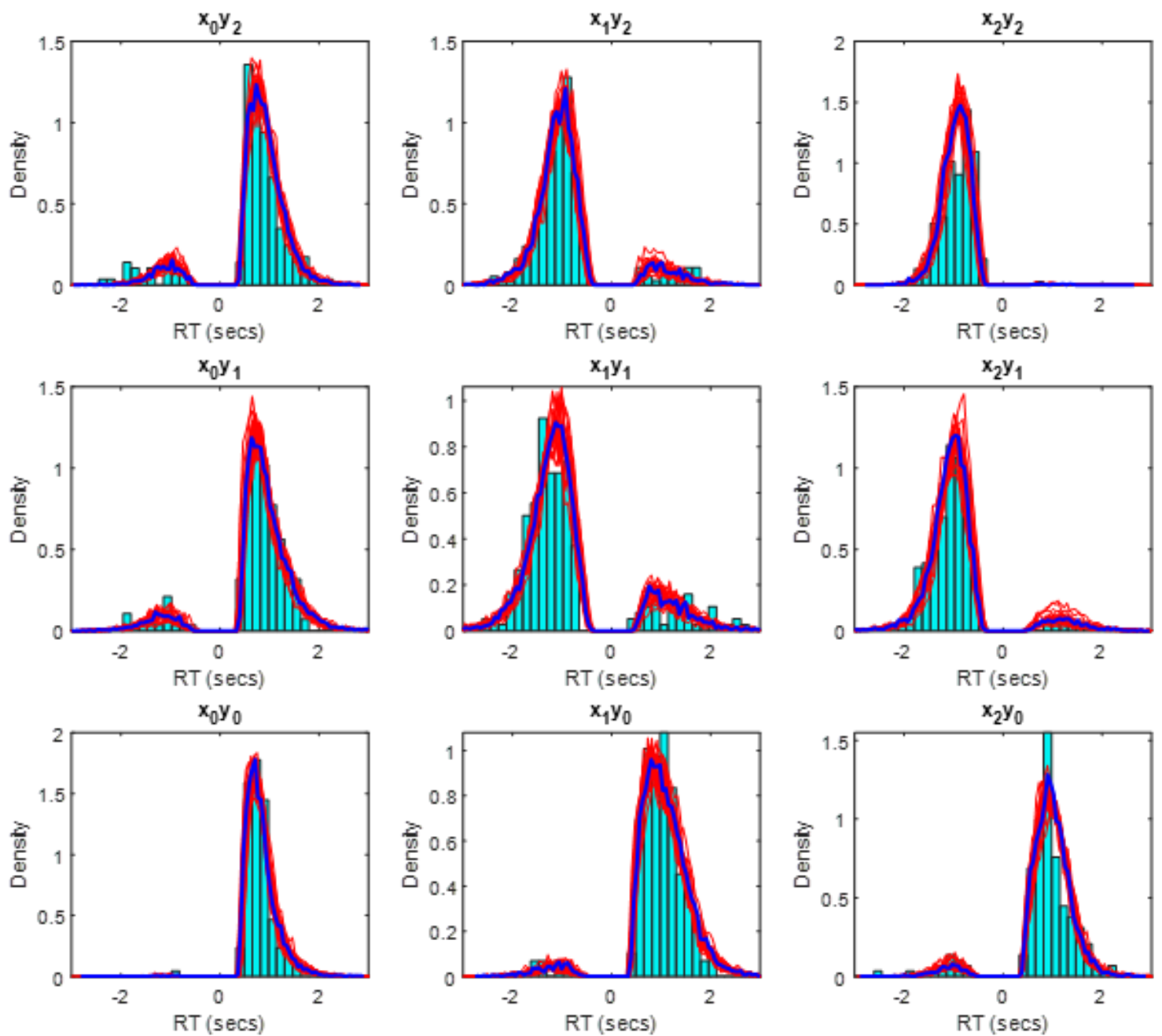

Figure 10. Posterior predictions from the fixed-variance serial self-terminating model for observer A3. Each subplot shows the predictions for one item. Positive RTs indicate "NO" responses (i.e., both items did not change). Negative RTs indicate a "YES" response (i.e., both items have changed). Data are plotted in the histogram. Each red line indicates a posterior sample. The solid blue line indicates the predictions using the average posterior parameter values. 

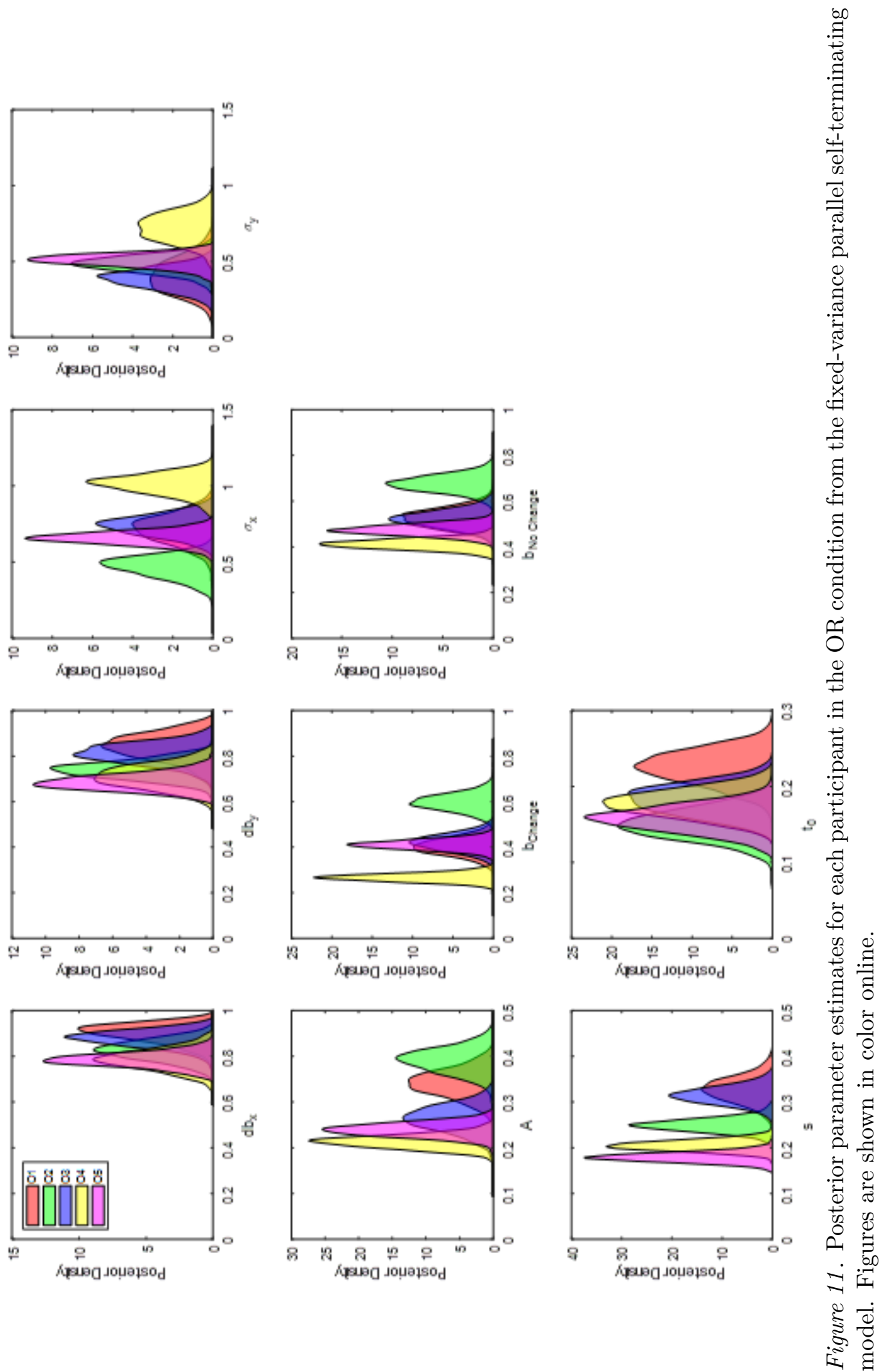

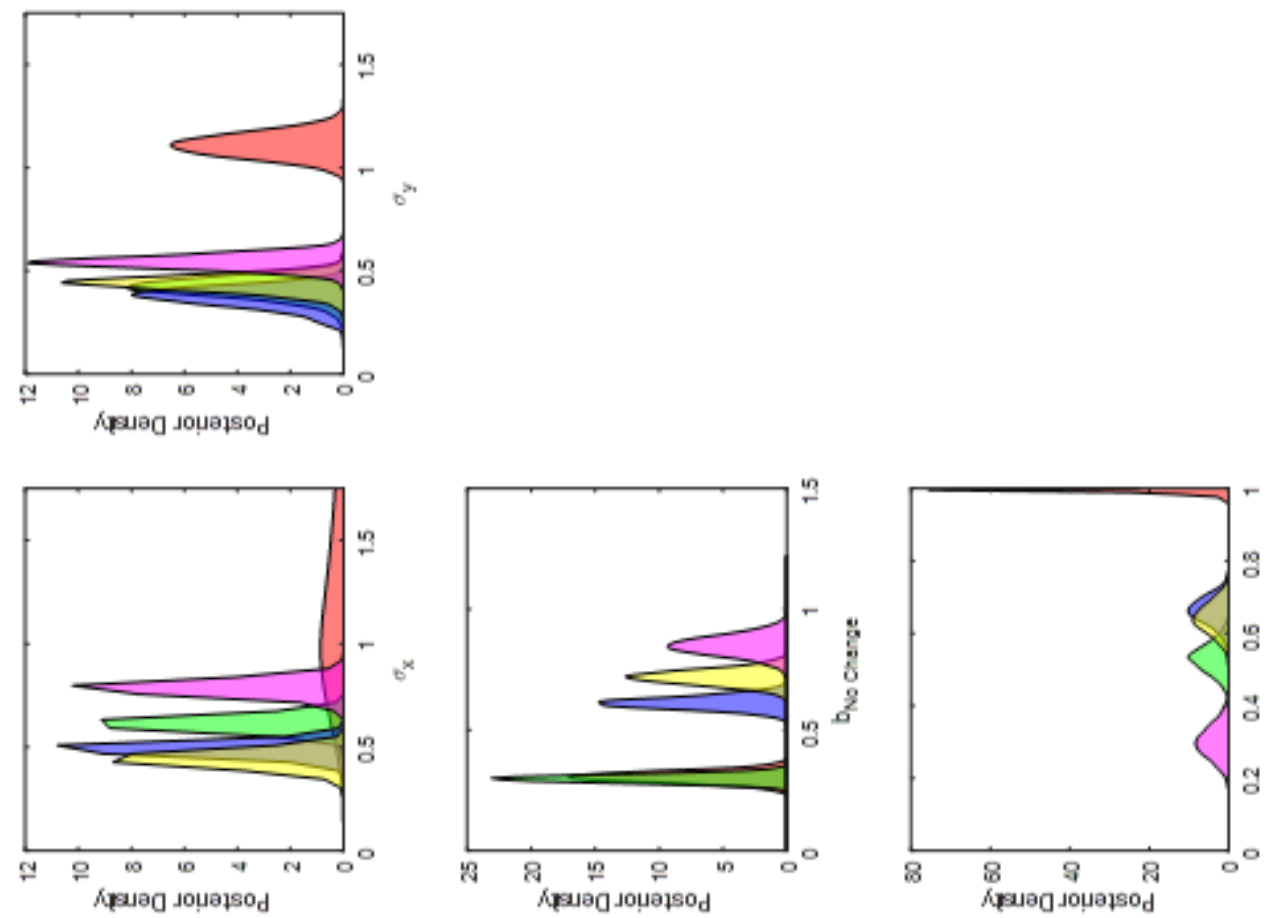

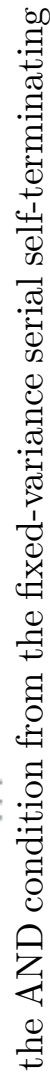
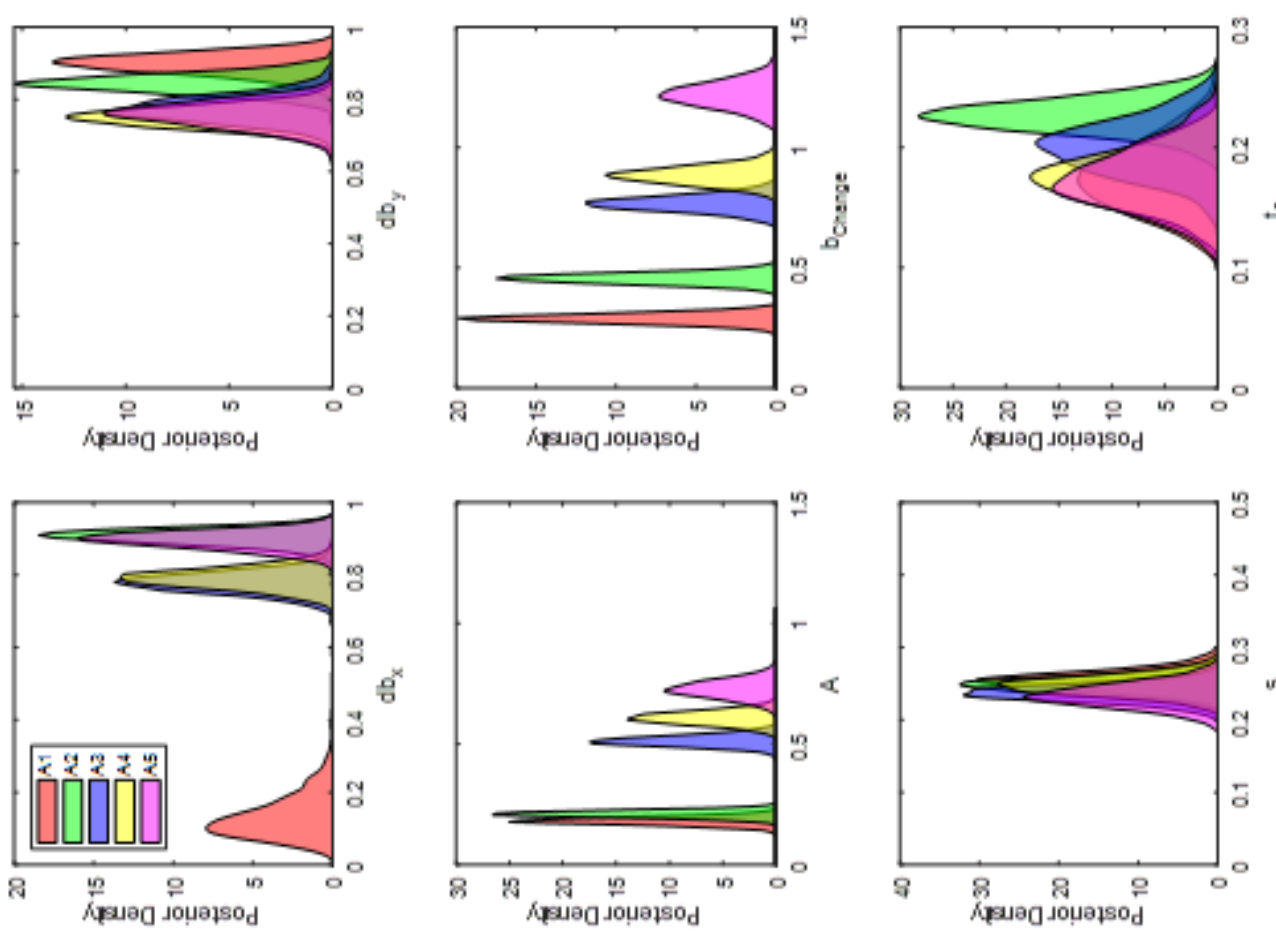

,

㭋 
model for most participants was the parallel self-terminating model. For the AND task, the best fitting model overall for three of the five participants (A2, A3, and A4) was the serial self-terminating model. Taken together, the evidence for the first-order coactive model is weaker than the evidence for models based on the independent accumulation of evidence at each location in both tasks. The different task demands of the OR task and the AND task appear to result in different attentional strategies.

Given the difference in architecture between OR and AND decision rules, a naturally arising question is whether the difference in strategy is also reflected by differences in the efficiency with which changes are detected. All of the participants in the OR task displayed capacity less than 1, and, hence, limited, but above the lower bound and consistent with the baseline parallel model. By contrast, in the AND task, two participants, A1 and A5, had capacity estimates which were greater than the upper bound for all $t$. These two participants were also best fit by the coactive-max model, which pools evidence from both locations. For the remaining AND participants, capacity tended to be greater than 1 but below the upper bound, again consistent with the parallel baseline model, despite the fact that the performance of these participants was best fit by a serial self-terminating model.

In Experiment 2, we pursued the specific question of how the efficiency of change detection varies as the number of possible changes in the display increases.

\section{Experiment 2}

In Experiment 2, we sought to compare the efficiency of change detection by comparing double targets to the case where single targets were presented with a second target which did not change (as in Experiment 1) and the case the single targets were presented in isolation. We term these conditions the single target + distractor condition and the single target alone condition. We utilized a redundant target change detection task in order to obtain measures of workload capacity in both cases. The stimuli were spatially separated luminance discs as in Experiment 1; however, we also incorporated trials where only a single item was presented (either on the left or right) in both probe and memory arrays.

Howard et al. (2020) recently presented evidence that capacity in the AND task can be misinterpreted when participants self-terminate on the "null" location instead of exhaustively processing both locations as intended by the design (see also Little et al., 2015). In our change detection task, this would arise as self-termination on the "same" location; consequently, we would not expect to see a difference between single target trials of different salience. In Experiment 1, we found that high salience single targets were not faster or more accurate than the low salience targets for most participants. One solution is the collect single target trials in a separate block as we do in our single target alone condition.

\section{Workload Capacity}

Increasing the number of features or objects that need to be processed before a decision can be made might increase, decrease, or leave unaffected the rate of processing changes (Townsend \& Ashby, 1983; Townsend, Fific, \& Neufeld, 2007; Wenger \& Townsend, 2000). Importantly, this differs from the common idea of item capacity often investigated in the change detection literature (see e.g. Luck \& Vogel, 1997) as it refers to limitations in 
processing rate, rather than a limit to the number of items held in memory. Workload capacity is also a continuous measure, measured as a function of time rather than a discrete item bottleneck.

In the redundant target detection task, two locations are monitored for the onset of stimuli or targets. Targets can appear in either of the locations, as well as both locations or neither location. In an OR design, participants respond affirmatively if any target is present; otherwise, participants respond negatively. In an AND design (see Figure 13), participants respond affirmatively only if both targets are present; otherwise, participants respond negatively. In the present change detection task, we implemented this design using possible changes from one display to the next rather than target onsets (cf., C.-T. Yang et al., 2014).

\section{Predictions}

Capacity in the OR task is measured using Equation 6, and capacity in the AND task is measured using Equation 8. The difference between the single target + distractor and single target alone condition is that different RTs are use for the single targets in both equations. Under simplifying assumptions of independence and context invariance, we might expect capacity to be unlimited in the OR task, in line with the finding that the architecture is consistent with parallel self-termination. By contrast, in the AND task, we might expect limited capacity since the architecture was consistent with serial exhaustive processing. On the other hand, there are theoretical indications that capacity might be limited. Lilburn (2016) showed that change detection decisions were consistent with the idea that a fixed number of samples were divided between the elements of the display. The implication is that the processing rate would be less efficient in the two item condition compared to the one item condition resulting in limited capacity. On the other hand, if processing is coactive and pooled, we might expect to see supercapacity. In Experiment 2, we examined capacity across the OR and AND conditions using a within-subjects design to determine whether the changes in capacity were due to changes in strategy across the tasks.

\section{Method}

Participants. Six individuals (4 F, 2 M; age range 25-29) with normal or corrected to normal vision (visual acuity of at least 20/25 as determined by a near-field Snellen eye chart; normal color vision as determined by Ishihara plates) from the University of Melbourne community participated in the study. Participants were reimbursed $\$ 10$ per session. One participant (C5), had also participated in Experiment 1 (as O1); the remaining participants were naïve to the experimental design.

Apparatus and Stimuli. The apparatus and stimuli were the same as in Experiment 1 with the addition of six single item stimuli comprising each left and right channel luminance level presented in isolation.

Procedure. The experiment was a one-shot change detection task in the same manner as Experiment 1 but with three conditions: OR, AND, and single-item. In the OR condition participants were asked to respond "different" if either one or the other target changed. In the AND condition participants were asked to respond with "different" only if 

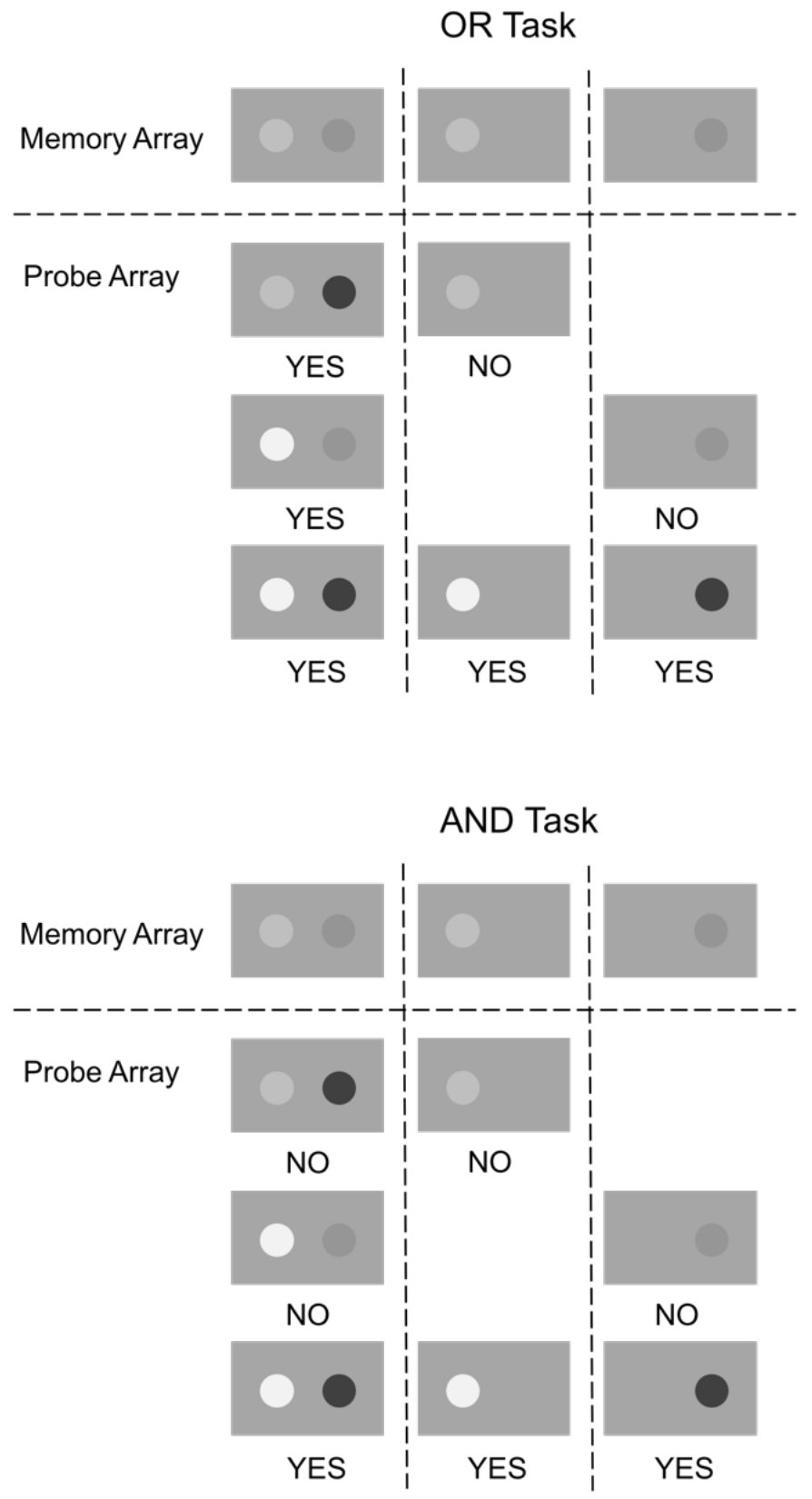

Figure 13. Example of redundant targets paradigm extended to a change detection task with an OR decision rule (top) and an AND decision rule (bottom). Note: as in Experiment 1 the memory and probe arrays varied and double change items could comprise no changes, one change, or two changes. 
both items had changed. The exact number of unique items and repetitions per block are printed in Table 11. Across both conditions, we ensured that change trials and same trials had equal frequency. In the single item condition, we presented the different trial types (High change, low changes, and same trials) and equal number of times.

Participants completed five experimental sessions (two OR sessions, two AND sessions, and one single-item session). Each session began with instructions explaining the response corresponding to the current condition followed by eight practice trials. Sessions consisted of six blocks of 48 trials each. The order of trials was randomized within each block. The order conditions were completed was also randomized.

Table 11

Trial frequencies per block and total presentation numbers for each trial type for Experiment 2.

\begin{tabular}{|c|c|c|c|c|c|c|c|c|c|}
\hline & & \multicolumn{8}{|c|}{ Item Conditions } \\
\hline & & $\mathrm{HH}$ & LL & HS & $\mathrm{LS}$ & $\mathrm{SH}$ & SL & SS & \\
\hline \multirow[t]{3}{*}{ OR } & Number of unique tokens & 4 & 4 & 4 & 4 & 4 & 4 & 8 & \\
\hline & Number of repetitions per block & 1 & 1 & 1 & 1 & 1 & 1 & 3 & \\
\hline & Total per session & 24 & 24 & 24 & 24 & 24 & 24 & 144 & \\
\hline \multirow[t]{4}{*}{ AND } & Number of unique tokens & 4 & 4 & 4 & 4 & 4 & 4 & 8 & \\
\hline & Number of repetitions per block & 3 & 3 & 1 & 1 & 1 & 1 & 1 & \\
\hline & Total per session & 72 & 72 & 24 & 24 & 24 & 24 & 48 & \\
\hline & & & & HX & LX & $\mathrm{XH}$ & $\mathrm{XL}$ & SX & $\mathrm{XS}$ \\
\hline \multirow[t]{3}{*}{ Single Items } & Number of unique tokens & & & 2 & 2 & 2 & 2 & 2 & 2 \\
\hline & Number of repetitions per block & & & 4 & 4 & 4 & 4 & 4 & 4 \\
\hline & Total per session & & & 72 & 72 & 72 & 72 & 72 & 72 \\
\hline
\end{tabular}

\section{Results}

RTs less than $200 \mathrm{~ms}$ or greater than $3000 \mathrm{~ms}$ were excluded from further analysis. Less than $1 \%$ of trials in total were removed using this method. Mean correct RTs, mean error RTs, and error rates for Experiment 2 are presented in Tables 12 to 14.

There was high consistency between participants in both the OR and AND conditions; for brevity, we report the median capacity functions for both the OR and AND conditions when capacity was computed both within condition (using the single target + distractor item conditions) and across conditions (using the single target alone condition; see Figure 14). The sole exception was the single target alone analysis of the AND condition; in this analysis, three participants showed limited capacity, two showed supercapacity, and one showed capacity near zero (see Table 15). Individual capacity estimates are reported in the supplementary material. Compared to the group, a participant might be faster or slower on either the redundant or single targets. To presented the group estimates, we therefore registered the RTs from all conditions by subtracting the median RT and adding the overall group median RT (see Burns, Houpt, Townsend, \& Endres, 2013). We additionally computed the median upper and lower bounds for each capacity estimate (i.e., the Race model 
Table 12

Mean Correct RTs (ms), Error RTs (ms) and Error Rates for the OR condition in Experiment 2.

\begin{tabular}{|c|c|c|c|c|c|c|}
\hline \multirow[b]{2}{*}{ Participant } & \multirow[b]{2}{*}{ Variable } & \multicolumn{5}{|c|}{ OR Condition } \\
\hline & & $\mathrm{HH}$ & $\mathrm{LL}$ & $\mathrm{HS} / \mathrm{SH}$ & $\mathrm{LS} / \mathrm{SL}$ & SS \\
\hline \multirow[t]{3}{*}{ C1 } & $\mathrm{RT}$ correct & 853 & 846 & 879 & 935 & 1036 \\
\hline & RT error & - & - & 1056 & 1156 & 1458 \\
\hline & $\mathrm{p}($ error $)$ & .00 & .00 & .25 & .43 & .10 \\
\hline \multirow[t]{3}{*}{$\mathrm{C} 2$} & RT correct & 923 & 1148 & 1042 & 1189 & 1090 \\
\hline & RT error & - & 1606 & 1271 & 1301 & 1390 \\
\hline & $\mathrm{p}$ (error) & .00 & .12 & .09 & .48 & .06 \\
\hline \multirow[t]{3}{*}{ C3 } & RT correct & 486 & 561 & 542 & 621 & 553 \\
\hline & RT error & - & 602 & 619 & 569 & 614 \\
\hline & $\mathrm{p}$ (error) & .00 & .21 & .14 & .43 & .27 \\
\hline \multirow[t]{3}{*}{$\mathrm{C} 4$} & RT correct & 703 & 914 & 768 & 995 & 780 \\
\hline & RT error & 465 & 986 & 652 & 1049 & 1447 \\
\hline & $\mathrm{p}$ (error) & .02 & .02 & .03 & .25 & .03 \\
\hline \multirow[t]{3}{*}{$\mathrm{C} 5$} & RT correct & 757 & 931 & 804 & 930 & 843 \\
\hline & RT error & 776 & 927 & 787 & 786 & 1008 \\
\hline & $\mathrm{p}$ (error) & .1 & .13 & .05 & .28 & .05 \\
\hline \multirow[t]{3}{*}{$\mathrm{C} 6$} & RT correct & 1131 & 1125 & 1171 & 1381 & 1228 \\
\hline & RT error & 1430 & 873 & 1201 & 1051 & 1251 \\
\hline & $\mathrm{p}$ (error) & .04 & .02 & .07 & .10 & .05 \\
\hline
\end{tabular}

Note: "-" indicates error free performance. 
Table 13

Mean Correct RTs (ms), Error RTs (ms) and Error Rates for the AND condition in Experiment 2.

\begin{tabular}{clccccc}
\hline \multirow{2}{*}{ Participant } & & \multicolumn{5}{c}{ AND Condition } \\
\cline { 3 - 6 } C1 & Variable & HH & LL & HS/SH & LS/SL & SS \\
& RT correct & 1129 & 1512 & 1474 & 1415 & 1365 \\
& RT error & 1658 & 1412 & 1337 & 1581 & 1586 \\
& p(error) & .07 & .47 & .34 & .20 & .01 \\
& RT correct & 1149 & 1488 & 1418 & 1410 & 1217 \\
& RT error & 1692 & 1610 & 1266 & 1445 & 1974 \\
& p(error) & .06 & .43 & .19 & .15 & .01 \\
C3 & RT correct & 670 & 791 & 725 & 733 & 711 \\
& RT error & 657 & 741 & 743 & 772 & 818 \\
& p(error) & .08 & .51 & .61 & .35 & .26 \\
C4 & RT correct & 956 & 1321 & 1292 & 1316 & 999 \\
& RT error & - & 1615 & 1152 & 1149 & 1345 \\
& p(error) & .00 & .17 & .14 & .12 & .03 \\
C5 & RT correct & 874 & 1008 & 918 & 845 & 761 \\
& RT error & 980 & 962 & 920 & 1052 & 1135 \\
& p(error) & .11 & .25 & .18 & .18 & .09 \\
C6 & RT correct & 1301 & 1753 & 1451 & 1531 & 1278 \\
& RT error & 1331 & 1743 & 1538 & 1659 & 1974 \\
& p(error) & .04 & .25 & .28 & .20 & .02 \\
\hline
\end{tabular}

Note: "-" indicates error free performance.

For the OR condition, capacity was below 1 both for the single target + distractor analysis and for the single target alone analysis; capacity tended to fall along the lower Grice boundary. For the AND condition, when computed with distractors, capacity was greater than 1 and above the upper boundary indicating supercapacity. By contrast, when computed using the single targets presented alone, capacity was less than 1 and below the lower bound when combined across the group.

We used Houpt and Townsend's (2012) null-hypothesis-significance test for the workload capacity analysis, which uses a z-test to make inferences about the capacity coefficient (see Table 15). If the Cz-statistic is negative then processing is limited in capacity, while if it is positive then related processing is supercapacity. In the OR condition, capacity was uniformly limited when compared with the baseline model, while in the AND condition performance across individuals super when computed within condition and was a mixture of limited and supercapacity when computed across conditions.

\section{Discussion}

Taken together, the capacity results suggest uniformly limited capacity processing for the OR task. For the AND task, the capacity with distractors was always super, but when presented with single targets alone, capacity was largely limited with some participants 
Table 14

Mean Correct RTs (ms), Error RTs (ms) and Error Rates for the Single Item condition in Experiment 2.

\begin{tabular}{clccccc}
\hline \multirow{2}{*}{ Participant } & & \multicolumn{5}{c}{ Single Item } \\
\cline { 3 - 6 } C1 & Variable & HX & LX & XH & XL & SX, XS \\
\hline \multirow{2}{*}{ C2 } & RT correct & 795 & 1088 & 679 & 690 & 1044 \\
& RT error & 1151 & 1343 & - & - & 1345 \\
& p(error) & .02 & .44 & .00 & .00 & .12 \\
& RT correct & 731 & 1022 & 707 & 792 & 760 \\
& RT error & 966 & 845 & 739 & 908 & 860 \\
C3 & p(error) & .04 & .44 & .02 & .06 & .15 \\
& RT correct & 503 & 529 & 478 & 524 & 606 \\
& RT error & 559 & 634 & - & 612 & 637 \\
C4 4 & p(error) & .12 & .54 & .00 & .17 & .29 \\
& RT correct & 886 & 1030 & 848 & 864 & 760 \\
& RT error & - & 1021 & - & 856 & 1426 \\
& p(error) & .00 & .21 & .00 & .15 & .07 \\
& RT correct & 920 & 1150 & 850 & 991 & 960 \\
& RT error & 829 & 1049 & 653 & 1325 & 1024 \\
& p(error) & .11 & .33 & .04 & .17 & .09 \\
C6 & RT correct & 1099 & 1322 & 941 & 1316 & 1321 \\
& RT error & - & 1779 & 967 & 1321 & 1686 \\
& p(error) & .00 & .17 & .02 & .06 & .13 \\
\hline
\end{tabular}

Note: "-" indicates error free performance.

exhibiting supercapacity. In both conditions, the results of the single targets + distractor analysis are commensurate with the capacity results from Experiment 1 . To interpret these results, we first consider the potential influence of stimulus and response contingencies.

Stimulus and Response Contingencies. Mordkoff and Yantis (1991) showed that when the value of a target in one location is predictable from the value of a nontarget in the other location, or when one response is predictable from the value of a non-target, various biases can accrue that can benefit redundant targets relative to the single targets. When the redundant target is benefited in a go/no-go redundant target detection task, violations of the race model inequality may be observed, but when these biases are absent, performance in the same task is consistent with the race model inequality (Mordkoff \& Miller, 1993; Mordkoff \& Yantis, 1991).

The amount of interstimulus contingency can be determined from the stimulus presentation frequencies. We first find the probability that the left location changes given that the right location stays the same, or that the left location is a target, $T$, given the right location is a non-target, $P\left(T_{L} \mid N_{R}\right)^{6}$. We then compute the interstimulus contingency, ISC, by subtracting the baseline probability a target ( $\mathrm{H}$ or $\mathrm{L}$ ) appearing on the left, $P\left(T_{L}\right)$,

\footnotetext{
${ }^{6}$ Our presentation frequencies did not vary by target location so the effect of the left non-target on the right is the same as the right non-target on the left.
} 

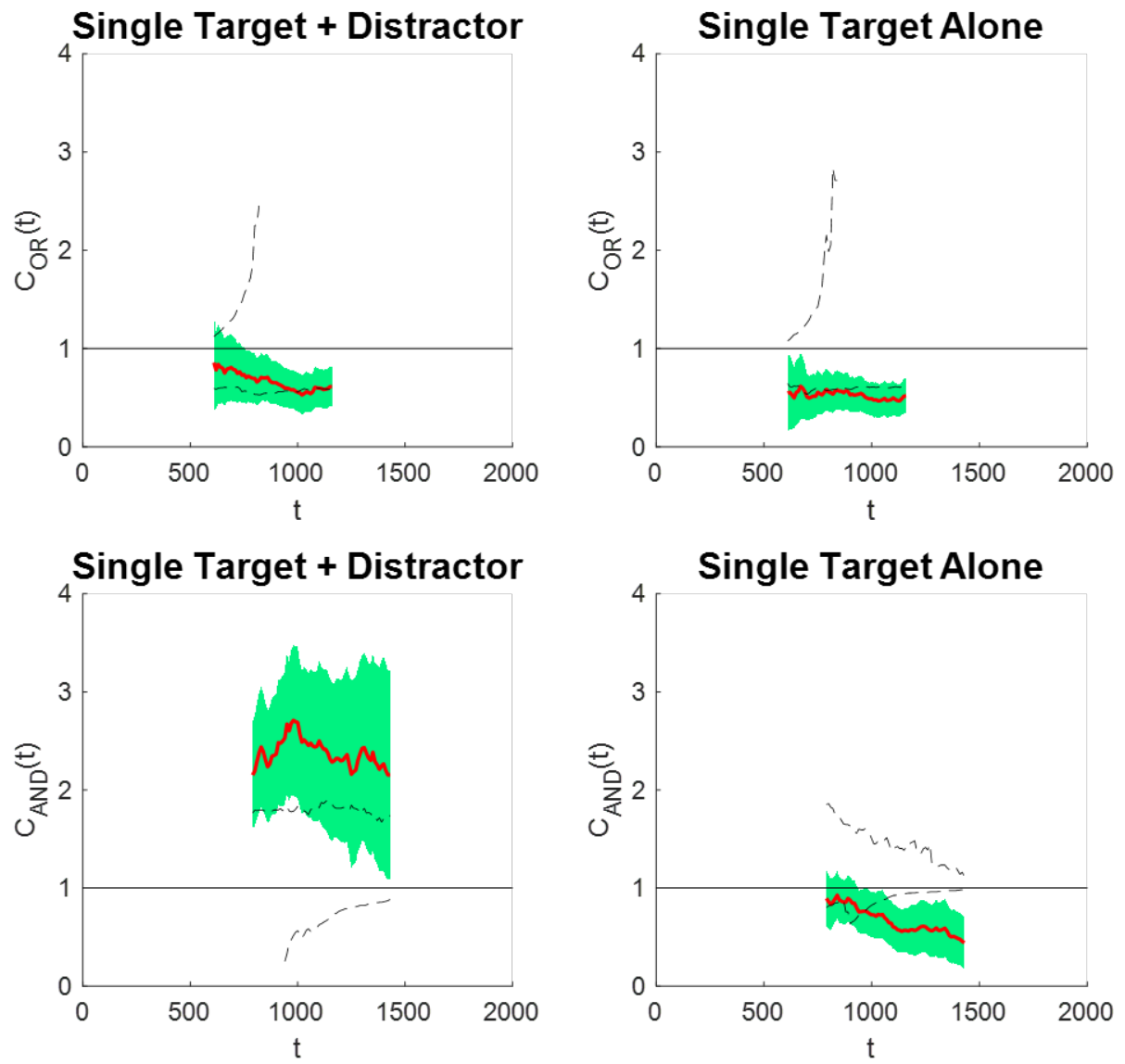

Figure 14. Median capacity estimates for each individual participant in the OR (top panels) and AND (bottom panels) conditions. Red line represents the capacity coefficient. Black dotted lines represent the Miller (upper) and Grice (lower) bounds, respectively. Left column: redundant target vs single targets+distractors. Right column: redundant target vs single targets alone.

$I S C\left(N_{R} \Rightarrow T_{L}\right)=P\left(T_{L} \mid N_{R}\right)-P\left(T_{L}\right)$. We also compute the ISC for a target on the left and a target on the right, $I S C\left(N_{R} \Rightarrow T_{L}\right)$. The redundant target benefit from an interstimulus contingency is given by subtracting the two:

$$
I S C B\left(N_{R}\right)=I S C\left(T_{R} \Rightarrow T_{L}\right)-I S C\left(N_{R} \Rightarrow T_{L}\right) .
$$

If Equation 11 is non-zero, then the value of the target channel is correlated with the value of the non-target channel. If Equation 11 is positive, then the information carried by the correlation favours the redundant targets over the single targets.

A second form of contingency bias, the non-target response contingency, is determined from the probability that a non-target (same) stimulus, $N$, predicts a target (change) response, + . Here the benefit for the redundant target is computed as $N R C B(N)=P(+)-P(+\mid N)$. If this value is positive, then there is a potential bias against responding with a change response for the single targets, which could lead to slower processing of the single targets, thus providing a benefit for redundant targets. If participants are able to track and make 
Table 15

Houpt-Townsend UCIP Test of Capacity for both AND and OR conditions

\begin{tabular}{|c|c|c|c|c|c|c|}
\hline \multirow[b]{2}{*}{ Participant } & \multicolumn{3}{|c|}{ OR: Single Targets + Distractors } & \multicolumn{3}{|c|}{ OR: Single Targets Alone } \\
\hline & Capacity & $\mathrm{z}$ & $\mathrm{p}$ & Capacity & $\mathrm{z}$ & $\mathrm{p}$ \\
\hline $\mathrm{C} 1$ & Non-significant & -0.22 & .83 & Limited & -5.59 & $<.001$ \\
\hline $\mathrm{C} 2$ & Limited & -2.15 & .03 & Limited & -8.84 & $<.001$ \\
\hline C3 & Non-significant & -1.61 & .11 & Limited & -4.24 & $<.001$ \\
\hline $\mathrm{C} 4$ & Limited & -2.94 & .003 & Limited & -3.17 & .001 \\
\hline C5 & Limited & -4.79 & $<.001$ & Non-significant & -1.81 & .07 \\
\hline \multirow[t]{2}{*}{ C6 } & Limited & -3.11 & .002 & Limited & -4.59 & $<.001$ \\
\hline & \multicolumn{3}{|c|}{ AND: Single Targets + Distractors } & \multicolumn{3}{|c|}{ AND: Single Targets Alone } \\
\hline Participant & Capacity & $\mathrm{z}$ & $\mathrm{p}$ & Capacity & $\mathrm{z}$ & $\mathrm{p}$ \\
\hline $\mathrm{C} 1$ & Super & 6.80 & $<.001$ & Limited & -5.88 & $<.001$ \\
\hline $\mathrm{C} 2$ & Super & 6.74 & $<.001$ & Limited & -8.76 & $<.001$ \\
\hline C3 & Super & 3.42 & $<.001$ & Limited & -3.06 & .002 \\
\hline $\mathrm{C} 4$ & Super & 8.46 & $<.001$ & Super & 3.29 & $<.001$ \\
\hline C5 & Super & 3.86 & $<.001$ & Super & 5.39 & $<.001$ \\
\hline C6 & Super & 4.04 & $<.001$ & Non-significant & 0.03 & .98 \\
\hline
\end{tabular}

use of these contingencies, then a positive ISCB and/or NRCB could lead to violation of the race-model inequality, which would imply supercapacity in our analysis (cf. Townsend \& Eidels, 2011).

Across all of our analyses, the ISCB and NRCB values were positive for Experiment 2: for the single target+distractor OR condition, $I S C B=.25$ and $N R C B=.25$; for the AND condition, $I S C B=.25$ and $N R C B=.5$. The results were similar for Experiment 1, which also used single target+distractors for the OR condition, $I S C B=.55$ and $N R C B=.33$, and the AND condition, $I S C B=.55$ and $N R C B=.29$. For the single target alone condition, these biases do not apply as one location contained a stimulus, but there was a bias toward responding "change", since targets appeared more than non-targets, which might have resulted in faster processing of the single targets relative to the comparative redundant targets. In sum, our single target+distractor analyses of Experiment 1 and 2 involved contingencies which, if relevant, should have facilitated redundant target processing leading to supercapacity. Our single target alone analyses involved biases, which should have favoured single targets, leading to limited capacity.

Despite these biases, we observed consistently limited capacity in the OR condition, which suggests that either responding was not affected by these contingencies or that there were other influences which exhibited a larger effect on response times. In the AND condition, we observed supercapacity for some participants, this could indicate a facilitatory effect of the contingences on response times in the single-target plus distractor analysis. On the other hand, the single target alone capacity in the AND condition could have been affected by the frequency of change responses in the single targets, which could have acted to push the capacity estimate below 1 , at least for three of the participants. 
There are a few reasons to suspect that the ISCB and NRCB may not have influenced responding in our task. For one, the effects of these contingencies have been demonstrated most conclusively in simple detection tasks involving Go/No-Go responding. Their influence has, to our knowledge, never been shown in a $2 \mathrm{AFC}$ change detection task. There are known differences between Go/No-Go and 2AFC tasks (Gomez, Ratcliff, \& Perea, 2007; Shenoy $\& \mathrm{Yu}, 2012)$. Primarily, the added time cost of withholding the No-Go response can lead to a Go bias (Shenoy \& Yu, 2012). Further, we are aware of no reports demonstrating an influence of the ISCB or NRCB on an AND detection task. It is beyond the scope of this paper to determine whether these contingencies affected capacity; however, the above caveats notwithstanding, we do note the possibility here at least for some participants in the AND condition. We can conclusively rule out a strong influence of these biases in the OR task since our observed capacity results are in the opposite direction.

Self-terminating on non-target location. An alternative explanation for supercapacity in the AND condition is that participants may be self-terminating on the locations which stay the same in single-target distractor displays, instead of processing the display exhaustively as expected by the baseline model. Howard et al. (2020) showed that if processing is exhaustive, then $\mathrm{H}$ single targets should be faster than $\mathrm{L}$ single targets. However, this relationship can break down if participants self-terminate on the unchanged location. As shown in Table 13, only three of six participants (C3, C4 and C6) have faster $\mathrm{H}$ single targets than L single targets. Further, none of these differences were significant (largest t-value was for C6, $t(180)=-1.00, p-.32$. (Howard et al., 2020) showed that in the case where processing self-terminates on the non-target channel in the AND task, then capacity will be greater than 1 if the non-target location is processed slower than the target location, that is, if deciding a location is the same is slower than deciding that a location has changed. We argue that this is a viable explanation for the supercapacity estimates that we observed in the AND task.

Howard et al. (2020) proposed a number of solutions to accurately diagnose capacity in the face of self-termination on the single targets. The first was to collect the single target data from a separate condition; this is what we have done in the single target alone analysis. The second suggestion was to use a new paradigm in which responses are collected for each type of stimulus in an identification task, where exhaustive processing is ensured. The data from this task can then be used to recover a more accurate estimate of capacity. We leave this as a target for future research.

\section{General Discussion}

In this paper, we sought to identify whether change-detection is best described by a first-order differencing integration model (Sorkin, 1962) or a second-order independent decisions integration model (Noreen, 1981; Shaw, 1982). We extended a set of change detection models, using different integration rules, to form a synthesized account of the time course of change detection. This account draws on prior work on mental architectures in categorization (Fifić et al., 2010) to provide a framework that allows for powerful non-parametric anlayses coupled with computational models of detection and response times. Our novel experimental method allows us to draw inferences about the processing architecture and capacity of detection decisions both when distractors are present and when they are absent. We further investigated how task requirements affect both architecture and capacity 
by utilizing disjunctive (OR) and conjunctive (AND) decision rules. We characterize the strategies of individual participants in line with the small-N approach (Smith \& Little, 2018).

Overall, our main empirical results were as follows: First, we found that changedetection decisions for the luminance level of two spatially separated discs generally occurred independently, consistent with second-order integration models for most participants. Second, we found that the task requirements affected processing strategy. Qualitatively, across both tasks, our non-parametric results agree with the predictions of the MAX model, which we extended to allow for serial exhaustive processing in the AND task by allowing for the maximum evidence source to determine the order of processing (see Figure 4). Quantitatively, for participants in the OR task, change detection decision-making was best described as parallel self-terminating in nature. This pattern of parallel self-terminating processing was consistent across both non-parametric, MIC and SIC results for the double change items, and parametric, computational modeling analyses. For the AND task, most of our participants showed that decision-making proceeded in serial, self-terminating fashion. Our modelling also showed some evidence that the change signal varied differentially as a function of the strength of the change. Our capacity analysis showed that in the AND tasks, participants self-terminate where possible when there is an absence of a change (cf. Howard et al., 2020; Little et al., 2015). For the OR task, there was little difference between the estimates when redundant targets were compared to single changes (i.e., when distractors were present) compared to single locations (i.e., when single targets were presented alone). Finally, setting aside the AND task results, the fifth major finding of the current work was that capacity tended to be limited in the OR task.

\section{Implications for Theories of Change Detection}

Our first major finding was that that change-detection decisions for the luminance level of two spatially separated used independent accumulation of evidence at each location in both tasks, consistent with second-order integration models for most participants. While Wilken and Ma (2004) ruled out a high threshold account of change detection decisionmaking, considering only choice probability meant it was difficult for their analysis to differentiate between first- and second-order accounts. This question has now been addressed in the current work.

Our second major finding that the attentional strategy (e.g., parallel in the OR task but serial in the AND task) changes as a function of the task is consistent with other findings in change-detection. For example, C.-T. Yang (2011) and C.-T. Yang et al. (2013, 2011) found that varying the probability and salience of a changes in dimensions of a single object affected the processing strategy used. Further, Donkin, Kary, Tahir, and Taylor (2016) found that when the environment was predictable (i.e., set size remained constant during each block), participants were able to flexibly allocate their attention across items. However, when the environment was unpredictable (i.e., set size varied from trial to trial) participants focused their attention on a smaller subset of items.

Within this context, studies of change detection often utilize set sizes of four item locations or more (Alvarez \& Cavanagh, 2004; Cowan, 2001; Donkin et al., 2016; Luck \& Vogel, 1997; Pashler, 1988; Phillips, 1974). In order to make further inferences regarding change detection decision-making in a one-shot change detection task, a worthwhile pursuit 
for future research would be investigating potential differences in the decision architecture for varying set-sizes in both OR and AND tasks. While it is well known that accuracy decreases with increasing set size (Lilburn, 2016; Luck \& Vogel, 1997; Pashler, 1988; Smith, 2016; Vogel, Woodman, \& Luck, 2006; Wilken \& Ma, 2004), especially above a limit of about four items (Cowan, 2001; Luck \& Vogel, 1997), it is unclear whether the processing architecture will change, reflecting a change in strategy for integrating information. Given change detection strategy has been shown to be sensitive to task demands (Donkin et al., 2016), a worthwhile pursuit for future research would be to test change detection performance in blocked and un-blocked experimental designs.

The third major finding of the current work was that workload capacity tends to be limited in the OR task. We consider three possible explanations for this results:

(1) The capacity analyses depends on an assumption of context invariance in order to draw conclusions about how efficiency varies compared to a baseline parallel model. The assumption of context invariance implies that the processing of a channel does not change based on the contents of the other channel. For example, the left location is assumed to be processed at the same rate regardless of the strength of the change in the right location including the case where nothing is presented on the right at all. If context invariance holds, then capacity can be limited simply because the processing architecture is not parallel as assumed by the baseline model. For instance, in the OR task, if processing is serial or exhaustive and context invariance holds, then capacity will be limited. In the AND task, if processing is serial, then capacity will be limited. In the present case, the limited capacity we observe in the OR task of Experiment 2 is not commensurate with the parallel selfterminating architecture inferred in Experiment 1.

(2) A second possibility is that there is a systematic violation of context invariance such that the processing rates for detecting changes in the redundant target are slower than processing rates for detecting changes in the corresponding location in the single targets. One explanation which predicts a systematic violation of context invariance is based on the idea that only a fixed amount of evidence or a fixed number of samples can be accumulated from a display in a given amount of time (Corbett \& Smith, 2017; Lilburn \& Smith, 2020; Palmer, 1990; Sewell et al., 2014). When set size is increased from one item to two items, the amount of samples is divided between two items leading to less efficient processing of each location with the larger set. In other words, the processing rate is slower when the sample size is higher. This would naturally violate the assumption of context invariance, consequently predicting limited capacity (see Altieri, Fifić, Little, \& Yang, 2017; Townsend, Liu, Zhang, \& Wenger, 2020, for a discussion of how violation of context invariance affects capacity). We discuss this sample size account in detail below, after discussing several empirical effects which can be elucidated by our findings.

(3) A third possibility for the OR task is that processing is parallel but that the parallel channels interact with each other. Eidels et al. (2011) investigated a series of parallel models which allowed for facilitatory and inhibitory interaction between the two channels. Inhibitory parallel models, in which one channel influences the other such that it slows evidence accumulation, can lead to limited capacity. Further, Townsend et al. (2020) showed that if context invariance holds, then interactions between channels can lead capacity to reverse across OR and AND decision rules. Although we observed such a reversal here, the fact that none of our AND subjects showed a difference between single targets of 
different salience suggests that this idea requires further empirical work. Nevertheless, an inhibitory parallel model is a plausible model of performance in the OR decision task. An example of this in practice is work by Smith and Sewell (2013) who proposed a model which uses parallel inhibitory interactions to capture the sample size model's effect in a dynamic fashion. We describe this model further below.

Our major findings that change detection is best described as a second-order model, that different tasks lead to different architectures, and that capacity is primarily limited with and without distractors, can inform several results in change detection and in the related literature.

Scene statistics in change-detection and change-blindness. Change blindness is a failure to notice otherwise perceptible changes in a visual environment when those changes are masked in some way (e.g., with an intervening blank screen or occlusions in the scene). This failure is thought to be associated with a lack of transient signals that would otherwise accompany a change (Rensink, O'Regan, \& Clark, 1997). One view suggests that change-blindness reflects a limit of visual attention (Rensink, 2000). Consequently, if changes occur in an attended region of space, then a comparison between the memory and visual scene can take place and changes are readily detected. The object or feature that has changed can then be identified. Such a finding accords well with the idea that processing occurs independently at different locations.

On the other hand, some studies have suggested that participants are able to use more general knowledge of the statistics of a scene to detect changes (Brady \& Tenenbaum, 2013; Howe \& Webb, 2014). For example, using a one-shot change detection paradigm which varied the proportion of green discs versus red discs, Howe and Webb (2014) showed that participants were accurately able to detect a change in the scene but were not always able to identify the location of the change. In a follow up experiment, Howe and Webb (2014) used the same number of individual item changes as the previous experiment but ensured that the overall proportion of colors remained the same between the displays. In this condition, participants were no longer able to accurately detect a change independently of identifying an item which had changed. This suggests that the underlying summary statistics (e.g., overall redness) were driving the detection of changes. In the latter experiment, however, the change had to be directly attended.

The finding that change detection performance can be affected by changes in scene statistics also suggests some implications for our understanding of processing architecture. In an OR design, where participants need to identify any change in the scene, it is possible that participants are simply monitoring the overall scene statistics in parallel. In the AND task, however, participants must instead verify that changes have occurred in both discs, resulting in a serial comparison. That serial analysis implies that attention must be applied to each location in the AND task.

A limitation of our current analysis is that more detailed differences in the overall composition of the scenes are not taken into account. By taking the absolute value of the perceptual distributions from the memory and probe arrays, we average across a number of potential different types of changes, assuming that the change magnitude for each disc (regardless of location or direction) have identical properties. For example, we treated $\mathrm{HH}$ trials where the left location became darker and the right location became lighter as identical to trials where both locations became darker. In the first example, the change in the two 
discs increases the overall range of luminance values present in the scene (i.e., the contrast) while the scene's mean luminance is left unaltered. In the second example, however, the contrast is unaltered while the mean luminance of the visual scene is decreased. A worthwhile direction for future research would be identifying whether or not changes which alter the overall scene statistics in these two ways alter the decision-making architecture. This could be accomplished by controlling the proportion of trials that changed in each manner.

Any-sameness and any-difference tasks. In a comparative visual search study, Taylor (1976) presented participants with two four-letter arrays side-by-side. In one condition, participants were asked was there any difference between the two arrays (i.e., an OR task). In the other condition, participants were asked if any of the items had remained the same (this is effectively an AND task since it is equivalent to asking have all items changed). Taylor (1976) found that in the any-difference task, RTs became shorter as the number of differences between the arrays (varying from one to four differences) increased. For their any-sameness condition, however, while the general pattern of RTs remained the same (RTs increased as the number of critical features decreased), the effect was much larger than in the any-difference task. Further, in the any-difference condition, there was an advantage for identical arrays, compared to arrays where only one or two items changed. However, when the critical number of features in the any-sameness condition was zero, there was no RT advantage. Taken together, these results suggest that any-sameness decisions are more difficult than any-difference decisions. These results would further be expected from a change in the decision strategy from parallel in the OR task to serial in the AND task.

Hyun, Woodman, Vogel, Hollingworth, and Luck (2009) further investigated the effects of any-sameness and any-difference decision rules in a change detection paradigm, hypothesizing that a change detection task would have a number of similarities to effects seen in visual search. In particular, they hypothesized that there would be a fundamental difference between the two decision-rules, as there is a fundamental difference in identifying presence versus absence of features (Taylor, 1976; Treisman, 1985). As in Taylor's (1976) comparative visual search task, Hyun et al. (2009) found that set size slopes for the anydifference condition were significantly shallower than set size slopes for the any-sameness condition, suggesting that detecting the absence of changes is more difficult than detecting the presence of changes. Additionally, both set size slopes were steeper than what would typically be expected for an unlimited capacity system. Again, these results would be expected if processing were parallel in the any-difference task but serial in the any-sameness task. Hyun and colleagues' finding that mean RT increased with set size even in the anydifference task is also commensurate with our finding of limited capacity in our OR task.

Hyun et al. (2009) looked in further detail at the locus of capacity limitations using the N2pc component which reflects the focusing of attention onto an object (Woodman \& Luck, 1999). They found that the latency of the N2pc component remained the same with varying set sizes, suggesting firstly, that changes attract spatial attention, and secondly, that identifying changes is unlimited in capacity. However, the P3 component (thought to represent a limited-capacity process that follows stimulus categorization; Kok, 2001) indicated a later limited-capacity comparison process. Hyun et al. (2009) suggested that this could be consistent with an effortful post-change-detection verification stage of all of the items. Alternatively, they suggested that the comparison process could be limited to the attended changes, but that this process becomes less efficient when more items are 
contained within visual short term memory (VSTM).

The double-target deficit. When attention must be divided between multiple sources of information, there are some small to moderate costs in detecting a target compared to focusing attention on a single source. However, when two targets which must be detected independently are presented simultaneously, there are large additional costs. This is commonly referred to as the double-target deficit and has been studied in the auditory literature (Moray, 1970a, 1970b; Sorkin \& Pohlmann, 1973; Sorkin, Pohlmann, \& Gilliom, 1973; Sorkin, Pohlmann, \& Woods, 1976) and later in visual perception (Corbett \& Smith, 2017; Duncan, 1980). Corbett and Smith (2017) suggested that the double-target deficit is caused by a capacity limitation in VSTM. This prediction relies on a two-stage system which can pre-attentively select targets while rejecting non-targets. The doubletarget deficit therefore arises because two target representations need to be formed in the double-target case but only one needs to be formed in the single-target case. Using a visual search task where participants searched for either one or two targets amongst three or two distractors, they found that this capacity limitation was well described by the sample-size relationship $1 / \sqrt{2}$ for the double-target deficit.

Burmester and Wallis (2012), like Wilken and Ma (2004), compared a low-threshold model with a high threshold (i.e., "slots-based") account. Specifically, they were interested in testing a sample-size account (Palmer, 1990; Sewell et al., 2014; Shaw, 1980), which assumes, like signal-detection theories, that stimuli are represented by a normally distributed noisy strength signal, that observers set a threshold for responding that can be exceeded based on noise alone, and that noise increases with the number of items which must be encoded in order to make a decision. The sample-size account has the additional constraint that a finite number of samples can be taken from the display in a given time period. If samples are taken uniformly from each location, then increasing the amount of relevant information in a visual scene necessarily decreases the quality of the samples. This process can be described by the equation $1 / \sqrt{m}$ where $\mathrm{m}$ is the number of stimuli relevant to a decision. Burmester and Wallis (2012) found that their sample-size model better accounted for participant choice probability in a change detection task varying changes in the color, speed, size, and orientation of Gabor patches, as well as in set size (1,2, 4, or 6 items) when compared to the high threshold, slot-based model. Sewell et al. (2014) also found that workload capacity limitations were well described by a sample-size relationship.

Smith and Sewell (2013) incorporated a sample-size relationship in their model of visual selection for briefly presented, multi-element visual displays. Using this relationship they characterized capacity limitations as occurring at the level of the visual representation in VSTM. In their model, the information retained by VSTM is determined by the selection and encoding stages which occur in parallel with competitive interaction. In Smith and Sewell (2013)'s model, when performing tasks such as visual search, items which contain task-relevant attributes or features excite the "where" pathway of attentional selection and in turn, mutually inhibit each other via competitive interaction. The excitation rate is modulated by attention. If the attention weights for items in VSTM are equivalent, the information capacity is described using a sample-size account $(1 / \sqrt{m}$ where $m$ is the number of items selected into VSTM). Their model therefore suggests that capacity limitations occur at the level of the visual representation, rather than, for example, at the decision-making stage. 
Our account is largely in agreement with their model, with the following exception: In their model, the decision-making stage is represented by a diffusion model accumulating noisy samples from this single, imperfect VSTM trace. In our account, the decision stage varies depending on the task demands. A diffusion model would be most consistent with a single channel model, like the MAX model, whereas we show here that processing is best accounted for by a parallel processing architecture in the OR task and a serial architecture in the AND task. This difference can be explained due to differences in experimental paradigm. For example, in Sewell et al. (2014) participants are asked to identify the value of a probed item presented after a to-be-remembered display is presented. In this paradigm, the probe display must be encoded into VSTM, but the decision only requires information from a single location unlike in our paradigm, where all locations are needed for the decision. The difference in decision architecture therefore maps closely to the task demands, but the fundamental sampling limitation of VSTM predict our corresponding limited capacity results.

\section{Conclusion}

In summary, the current work provides novel insight into the integration of information in change detection decision-making by accounting for the time course of information processing. This was achieved through the synthesis of the mental architectures approache (Fifić et al., 2010) with signal detection theories of change detection (Wilken \& Ma, 2004). In particular, we made the novel finding that for most participants change detection decisionmaking is best described by a second-order integration model whereby decisions are made about each stimulus independently. Furthermore, we found that the task requirements affected processing strategy. When change detection decisions involved a disjunctive rule (OR task), decision-making was best described as a parallel self-terminating process. When change detection decisions involved a conjunctive rule (AND task), decision-making was generally best described as a serial self-terminating process, although there were some individual differences. Taken together with our findings of limited capacity in both tasks, our strong inference method allows us to rule out a large number of different hypotheses and to provide a basis which can inform future modeling of change detection decisions using RT. Lastly, we find our results in accord with recent theorizing from related domains, and we hope that our results are, in conjunction, mutually informative. These findings provide a way-point for moving forward in the study of change detection. In particular we have identified a number of important avenues for future study, the most important of which are a) examining whether and how architecture changes with set size in both the OR and AND tasks b) examining whether changing the predictability of the number of changes affects processing architecture and c) examining whether changes affecting the overall scene statistics changes processing architecture. 
References

Akaike, H. (1974). A new look at the statistical model identification. IEEE Transactions on Automatic Control, 19, 716-723.

Altieri, N., Fifić, M., Little, D. R., \& Yang, C.-T. (2017). A tutorial introduction and historical background to systems factorial technology. In D. R. Little, N. Aliteri, M. Fifić, \& C.-T. Yang (Eds.), Systems factorial technology: A theory driven methodology for the identification of perceptual and cognitive mechanisms. Elsevier.

Alvarez, G. A., \& Cavanagh, P. (2004). The capacity of visual short-term memory is set both by visual information load and by number of objects. Psychological Science, 15(2), 106-111.

Ashby, F. G., \& Gott, R. E. (1988). Decision rules in the perception and categorization of multidimensional stimuli. Journal of Experimental Psychology: Learning, Memory and Cognition, $14,33-53$.

Ashby, F. G., \& Townsend, J. T. (1986). Varieties of perceptual independence. Psychological Review, 93(2), 154-179-.

Bays, P. M., \& Husain, M. (2008). Dynamic shifts of limited working memory resources in human vision. Science, 321 (5890), 851-854.

Blunden, A. G., Howe, P. D. L., \& Little, D. R. (2020). Evidence that within-dimension features are generally processed coactively. Attention, Perpection, \&3 Psychophysics, 82(1), 193-227.

Blunden, A. G., Wang, T., Griffiths, D., \& Little, D. (2015). Logical-rules and the classification of integral dimensions: Individual differences in the processing of arbitrary dimensions. Frontiers in Psychology, 5.

Brady, T. F., \& Tenenbaum, J. B. (2013). A probabilistic model of visual working memory: Incorporating higher order regularities into working memory capacity estimates. Psychological Review, 120, 85-109.

Brainard, D. (1997). The psychophysics toolbox [Journal Article]. Spatial Vision, 10, 433-436.

Brown, S. D., \& Heathcote, A. (2008). The simplest complete model of choice response time: Linear ballistic accumulation. Cognitive Psychology, 57, 153-178.

Burmester, A., \& Wallis, G. (2012). Contrasting predictions of low- and high-threshold models for the detection of changing visual features. Perception, 41, 505-516.

Burns, D., Houpt, J. W., Townsend, J. T., \& Endres, M. J. (2013). Functional principle components analysis of workload capacity functions [Journal Article]. Behavior Research Methods, 45, 1048-1057.

Busemeyer, J. R. (1985). Decision making under uncertainty: A comparison of simple scalability, fixed-sample, and sequential-sampling models. Journal of Experimental Psychology: Learning, Memory and Cognition, 11, 538-564.

Bushmakin, M., Eidels, A., \& Heathcote, A. (2015). Breaking the rules in perceptual information integration. In Presented at the meeting of the mathematical psychology society.

Chang, T.-Y., Little, D. R., \& Yang, C.-T. (2016). Selective attention modulates the effect of target location probability on redundant signal processing. Attention, Perception, $E_{3}$ Psychophysics, $78,1603-1624$.

Chechile, R. A. (2003). Mathematical tools for hazard function analysis. Journal of Mathematical Psychology, 47, 478-494.

Chechile, R. A. (2011). Properties of reverse hazard functions. Journal of Mathematical Psychology, $55,203-222$.

Cheng, X. J., McCarthy, C. J., Wang, T. S., Palmeri, T. J., \& Little, D. R. (2018). Composite faces are not (necessarily) processed coactively: A test using systems factorial technology and logical-rule models. Journal of Experimental Psychology: Learning, Memory and Cognition, $44(6), 833$.

Colonius, H., \& Vorberg, D. (1994). Distribution inequalities for parallel models with unlimited capacity. Journal of Mathematical Psychology, 38, 35-58. 
Corbett, E. A., \& Smith, P. L. (2017). The magical number one-on-square-root-two: The doubletarget deficit in brief visual displays. Journal of Experimental Psychology: Human Perception and Performance, 43(7), 1376-1369.

Cowan, N. (2001). The magical number 4 in short-term memory: A reconsideration of mental storage capacity. Behavioral and Brain Sciences, 24(1), 87-114.

Cowan, N., \& Rouder, J. N. (2009). Comment on "dynamic shifts of limited working memory resources in human vision". Science, 323(5916), 877.

Dai, H., Versfeld, N. J., \& Green, D. M. (1996). The optimum decision rules in the same-different paradigm. Perception 83 Psychophysics, 58(1), 1-9.

Donkin, C., Kary, A., Tahir, F., \& Taylor, R. (2016). Resources masquerading as slots: Flexible allocation of visual working memory. Cognitive Psychology, 85, 30-42.

Donkin, C., Nosofsky, R. M., Gold, J. M., \& Shiffrin, R. M. (2013). Discrete-slots models of visual working-memory response times. Psychological Review, 120, 873-902.

Duncan, J. (1980). The locus of interference in the perception of simultaneous stimuli. Psychological Review, 87, 272-300.

Dzhafarov, E. N. (2003). Selective influence through conditional independence. Psychometrika, 68, $7-26$.

Eidels, A., Houpt, J. W., Altieri, N., Pei, L., \& Townsend, J. T. (2011). Nice guys finish fast and bad guys finish last: Facilitatory vs. inhibitory interaction in parallel systems. Journal of Mathematical Psychology, 55, 176-190.

Fifić, M., Little, D. R., \& Nosofsky, R. (2010). Logical-rule models of classification response times: A synthesis of mental-architecture, random-walk, and decision-bound approaches. Psychological Review, 117, 309-348.

Fifić, M., Nosofsky, R. M., \& Townsend, J. (2008). Information-processing architectures in multidimensional classification: A validation of test of the systems factorial technology. Journal of Experimental Psychology: Human Perception \&3 Performance, 34, 356-375.

Fox, E. L., \& Houpt, J. W. (2016). The perceptual processing of fused multi-spectral imagery. Cognitive Research: Principles and Implications, 1(31). Retrieved from $10.1186 / \mathrm{s} 41235-016-0030-7$

Gardner, G. T. (1973). Evidence for independent parallel channels in tachistoscopic perception. Cognitive Psychology, 4(1), 130-155.

Gelman, A., Hwang, J., \& Vehtari, A. (2014). Understanding predictive information criteria for bayesian models. Statistics and Computing, 24(6), 997-1016.

Gomez, P., Ratcliff, R., \& Perea, M. (2007). A model of the go/no-go task. Journal of Experimental Psychology: General, $136(3), 389$.

Graham, N., Kramer, P., \& Yager, D. (1987). Signal-detection models for multidimensional stimuli: Probability distributions and combination rules. Journal of Mathematical Psychology, 31(4), 366-409.

Grice, G. R., Canham, L., \& Gwynne, J. W. (1984). Absence of a redundant-signals effect in a reaction time task with divided attention. Perception \&3 Psychophysics, 36, 565-570.

Griffiths, D. W., Blunden, A. G., \& Little, D. R. (2017). Logical-rule based models of categorization: Using systems factorial technology to understand feature and dimensional processing. In D. R. Little, N. Aliteri, M. Fifić, \& C.-T. Yang (Eds.), Systems factorial technology: A theory driven methodology for the identification of perceptual and cognitive mechanisms (p. 245-269). Academic Press.

Heathcote, A., Brown, S. D., \& Wagenmakers, E.-J. (2015). An introduction to good practices in cognitive modeling. In An introduction to model-based cognitive neuroscience (pp. 25-48). Springer.

Hefner, R. A. (1958). Extensions of the law of comparative judgment to discriminable and multidimensional stimuli (Unpublished doctoral dissertation). University of Michigan.

Holmes, W. R. (2015). A practical guide to the probability density approximation (pda) with 
improved implementation and error characterization. Journal of Mathematical Psychology, 68-69, 13-24.

Houpt, J. W., Blaha, L. M., McIntire, J. P., Havig, P. R., \& Townsend, J. T. (2014). Systems Factorial Ttechnology with R. Behavior Research Methods, 46, 307-330.

Houpt, J. W., \& Little, D. R. (2017). Statistical analysis of the resilience function. Behavior Research Methods, 49, 1261-1277.

Houpt, J. W., \& Townsend, J. T. (2010). The statistical properties of the survivor interaction contrast. Journal of Mathematical Psychology, 54, 446-453.

Houpt, J. W., \& Townsend, J. T. (2011). An extension of sic predictions to the weiner coactive model. Journal of Mathematical Psychology, 55, 267-270.

Houpt, J. W., \& Townsend, J. T. (2012). Statistical measures for workload capacity analysis. Journal of Mathematical Psychology, 56, 341-355.

Howard, Z. L., Garrett, P., Little, D. R., Townsend, J. T., \& Eidels, A. (2020). A show about nothing: No-signal processes in systems factorial technology. Psychological Review. Retrieved from psyarxiv.com/b98wn (Accepted 29-Jun-2020)

Howe, P. D., \& Webb, M. E. (2014). Detecting unidentified changes. PLoS-ONE, 9(1), e84490.

Hull, C. L. (1943). Principles of behavior. New York: Appleton-century-crofts.

Hyun, J. S., Woodman, G. F., Vogel, E. K., Hollingworth, A., \& Luck, S. J. (2009). The comparison of visual working memory representations with perceptual inputs. Journal of Experimental Psychology: Human Perception and Performance, 35(4), 1140-1160.

Kantowitz, B. H. (1974). Human information processing: Tutorials in performance and cognition. Oxford, England: Lawrence Erlbaum.

Kleiner, M., Brainard, D., Pelli, D., Ingling, A., Murray, R., Broussard, C., \& et al. (2007). WhatâẮ́s new in psychtoolbox-3. Perception, 36, 1.

Kok. (2001). On the utility of the p3 amplitude as a measure of processing capacity. Psychophysiology, 38, 557-577.

Kool, W., McGuire, J. T., Rosen, Z. B., \& Botvinick, M. M. (2010). Decision making and the avoidance of cognitive demand. Journal of Experimental Psychology: General, 139(4), 665682.

Koopman, B. O. (1956). The theory of search. Operations Research, 4, 5.

Leone, F. C., Nelson, L. S., \& Nottingham, R. B. (1961). The folded normal distribution. Technometrics, 3(4), 543-550.

Li, X., Liang, Z., Kleiner, M., \& Lu, Z.-L. (2010). Rtbox: A device for highly accurate response time measurements. Behavior Research Methods, 42(1), 212-225.

Lilburn, S. D. (2016). Information limits within visual short term memory (Unpublished doctoral dissertation). The University of Melbourne.

Lilburn, S. D., \& Smith, P. L. (2020). A single, simple, statistical mechanism explains resource distribution and temporal updating in visual short-term memory. Cognitive Psychology, 122, 101330.

Link, S. W. (1992). The wave theory of difference and similarity. Psychology Press.

Little, D. R., Altieri, N., Fifić, M., \& Yang, C.-T. (2017). Systems factorial technology: A theory driven methodology for the identification of perceptual and cognitive mechanisms. New York: Academic Press.

Little, D. R., Eidels, A., Fific, M., \& Wang, T. (2015). Understanding the influence of distractors on workload capacity. Journal of Mathematical Psychology, 68, 25-36.

Little, D. R., Eidels, A., Fific, M., \& Wang, T. (2018). How do information processing systems deal with conflicting information? differential predictions for serial, parallel and coactive processing models. Computational Brain \& Behavior, 1, 1-21.

Little, D. R., Eidels, A., Houpt, J. W., \& Yang, C. T. (2017). Set size slope still does not distinguish parallel from serial search. Behavioral and Brain Sciences, 40,32-33.

Little, D. R., Nosofsky, R., \& Denton, S. E. (2011). Response time tests of logical rule-based models 
of categorization. Journal of Experimental Psychology: Learning, Memory and Cognition, 37, $1-27$.

Little, D. R., Nosofsky, R. M., Donkin, C., \& Denton, S. E. (2013). Logical-rules and the classification of integral dimensioned stimuli. Journal of Experimental Psychology: Learning, Memory and Cognition, 39, 801-820.

Luce, R. D. (1986). Response times: Their role in inferring elementary mental organization. New York: Oxford University Press.

Luck, S., \& Vogel, E. K. (1997). The capacity of visual working memory for features and conjunctions. Nature, 390 (6657), 279-81.

Macmillan, N. A., \& Creelman, C. D. (2004). Detection theory: A user's guide. Psychology Press.

Miller, J. (1982). Divided attention: Evidence for coactivation with redundant signals. Cognitive Psychology, 14, 247-279.

Moneer, S., Wang, T., \& Little, D. R. (2016). The processing architectures of whole-object features: A logical rules approach. Journal of Experimental Psychology: Human Perception and Performance, 43, 1443-1465.

Moray, N. (1970a). Introductory experiments in auditory time sharing: Detection of intensity and frequency increments. Journal of the Acoustical Society of America, 47, 1071-1073.

Moray, N. (1970b). Time sharing in auditory detection: Effects of stimulus duration. Journal of the Acoustical Society of America, 47, 660-661.

Mordkoff, J. T., \& Miller, J. (1993). Redundancy gains and coactivation with two different targets: The problem of target preferences and the effects of display frequency. Perception $\&$ Psychophysics, 53, 527-535.

Mordkoff, J. T., \& Yantis, S. (1991). An interactive race model of divided attention. Journal of Experimental Psychology: Human Perception and Performance, 17, 520.

Noreen, D. L. (1981). Optimal decision rules for some common psychophysical paradigms. In S. Grossberg (Ed.), Mathematical psychology and psychophysiology (p. 237-280). Providence, RI: Psychology Press.

Palmer, J. (1990). Attentional limits on the perception and memory of visual information. Journal of Experimental Psychology: Human Perception and Performance, 16(2), 1227-1268.

Pashler, H. (1988). Familiarity and visual change detection. Current Directions in Psychological Science, 4(4), 369-378.

Pelli, D. (1997). The videotoolbox software for visual psychophysics: Transforming numbers into movies [Journal Article]. Spatial Vision, 10, 437-442.

Phillips, W. A. (1974). On the distinction between sensory storage and short-term visual memory. Attention, Perception, Es Psychophysics, 16(2), 283âĂŞ290.

Raab, D. (1962). Statistical facilitation of simple reaction time. Transaction of the New York Academy of Science, 43, 574-590.

Ratcliff, R. (1978). A theory of memory retrieval. Psychological Review, 85, 59-108.

Ratcliff, R., \& Rouder, J. N. (1998). Modeling response times for two-choice decisions. Psychological Science, 5, 347-356.

Rensink, R. A. (2000). Visual search for change: A probe into the nature of attentional processing. Visual Cognition, 7(1-3), 345-376.

Rensink, R. A., O'Regan, J. K., \& Clark, J. J. (1997). To see or not to see: The need for attention to perceive changes in scenes. Psychological Science, 8(5), 368-373.

Schweickert, R. (1992). Information, time, and the structure of mental events: A twenty-five year review. In D. E. Meyer \& S. Kornblum (Eds.), Attention and performance: Vol. 14. synergies in experimental psychology, artificial intelligence, and cognitive neuroscience - a silver jubilee (p. 535-566). Cambridge, MA: MIT Press.

Schweickert, R., Fisher, D. L., \& Sung, K. (2012). Discovering cognitive architecture by selectively influencing mental processes (Vol. 4). World Scientific.

Sewell, D. K., Lilburn, S. D., \& Smith, P. L. (2014). An information capacity limitation of visual 
short-term memory. Journal of Experimental Psychology: Human Perception and Performance, 40(6), 2214-2242.

Shaw, M. L. (1980). Identifying attentional and decision-making components in information processing. In R. S. Nickerson (Ed.), Attention and performance viii (p. 277-295). Hillsdale, NJ: Lawrence Erlbaum Associates.

Shaw, M. L. (1982). Attending to multiple sources of information. Cognitive Psychology, 14, 353-409.

Shenoy, P., \& Yu, A. J. (2012). Strategic impatience in go/nogo versus forced-choice decision-making. Advances in Neural Information Processing Systems, 25, 2123-2131.

Smith, P. L. (1998). Attention and luminance detection: A quantitative analysis. Journal of Experimental Psychology: Human Perception and Performance, 24(1), 105-133.

Smith, P. L. (2010). Spatial attention and the detection of weak visual signals. In V. Coltheart (Ed.), Tutorials in visual cognition (p. 211-259). Psychology Press.

Smith, P. L. (2016). Diffusion theory of decision making in continuous report. Psychological Review, $123(4), 425-451$.

Smith, P. L., \& Little, D. R. (2018). Small is beautiful: In defence of the small- $N$ design. Psychonomic Bulletin \& Review, 25(6), 2083-2101.

Smith, P. L., \& Sewell, D. K. (2013). A competitive interaction theory of attentional selection and decision making in brief, multielement displays. Psychological Review, 120(3), 589-627.

Sorkin, R. D. (1962). Extension of the theory of signal detectability to matching procedures in psychoacoustics. Journal of the Acoustical Society of America, 34, 1945-1751.

Sorkin, R. D., \& Pohlmann, L. D. (1973). Some models of observer behavior in two-channel auditory signal detection. Perception \&3 Psychophysics, 53, 1045-1050.

Sorkin, R. D., Pohlmann, L. D., \& Gilliom, J. (1973). Simultaneous two-channel signal detection: Iii. 630- and 1400-hz signals. Journal of the Acoustical Society of America, 53, 1045-1050.

Sorkin, R. D., Pohlmann, L. D., \& Woods, D. D. (1976). Decision interaction between auditory channels. Perception \& Psychophysics, 19, 290-295.

Sternberg, S. (1969). Memory scanning: Memory processes revealed by reaction-time experiments. American Scientist, 4, 421-457.

Swets, J. A. (1961). Is there a sensory threshold? Science, 134(3473), 168-177.

Swets, J. A., Tanner, W. P., \& Birdsall, T. G. (1961). Decision processes in perception. Psychological Review, 68(5), 301-340.

Tanner, W. P., \& Swets, J. A. (1954a). A decision-making theory of visual detection. Psychological Review, 61(6), 401-409.

Tanner, W. P., \& Swets, J. A. (1954b). The human use of information-i: Signal detection for the case of the signal known exactly. Transactions of the IRE Professional Group on Information Theory, 4 (4), 213-221.

Taylor, D. A. (1976). Effect of identity in the multiletter matching task. Journal of Experimental Psychology: Human Perception and Performance, 20, 199-806.

Thiele, J. E., Haaf, J. M., \& Rouder, J. N. (2017). Is there variation across individuals in processing? Bayesian analysis for systems factorial technology. Journal of Mathematical Psychology, 81, 40-54. Retrieved from psyarxiv.com/auf9n

Townsend, J. T. (1972). Some results concerning the identifiability of parallel and serial processes. British Journal of Mathematical and Statistical Psychology, 25, 168-199.

Townsend, J. T. (1984). Uncovering mental processes with factorial experiments. Journal of Mathematical Psychology, 28, 363-400.

Townsend, J. T. (1990a). Serial vs. parallel processing: Sometimes they look like tweedledum and tweedledee but they can (and should) be distinguished. Psychological Science, 1, 46-54.

Townsend, J. T. (1990b). Truth and consequences of ordinal differences in statistical distributions: Toward a theory of hierarchical inference. Psychological Bulletin, 108, 551.

Townsend, J. T., \& Ashby, F. G. (1983). The stochastic modeling of elementary psychological 
processes. Cambridge: Cambridge University Press.

Townsend, J. T., \& Eidels, A. (2011). Workload capacity spaces: A unified methodology for response time measures of efficiency as workload is varied. Psychonomic Bulletin E Review, $18,659-681$.

Townsend, J. T., Fific, M., \& Neufeld, R. W. (2007). Assessment of mental architecture in clinical/cognitive research. In T. A. Treat, R. R. Bootzin, \& T. B. Baker (Eds.), Psychological clinical science: Papers in honour of richard m. mcfall (p. 223-258). Mahwah, NJ: Lawrence Erlbaum Associates.

Townsend, J. T., Houpt, J. W., \& Silbert, N. H. (2012). General recognition theory extended to include response times: Predictions for a class of parallel systems. Journal of Mathematical Psychology, 56 (6), 476-494.

Townsend, J. T., Liu, Y., Zhang, R., \& Wenger, M. J. (2020). Interactive parallel models: No virginia, violation of millerâÁŹs race inequality does not imply coactivation and yes virginia, context invariance is testable. The Quantitative Methods for Psychology, 16, 192-212.

Townsend, J. T., \& Nozawa, G. (1995). Spatio-temporal properties of elementary perception: An investigation of parallel, serial and coactive theories. Journal of Mathematical Psychology, 39, 321-340.

Townsend, J. T., \& Schweickert, R. (1989). Toward the trichotomy method of reaction times: Laying the foundation of stochastic mental networks. Journal of Mathematical Psychology, 33(3), 309-327.

Townsend, J. T., \& Wenger, M. J. (2004). A theory of interactive parallel processing: New capacity measures and predictions for a response time inequality series. Psychological Review, 111, 1003-1035.

Treisman, M. (1985). The magical number seven and some other features of category scaling: Properties of a model for absolute judgment. Journal of Mathematical Psychology, 29(2), $175-230$.

Turner, B. M., \& Sederberg, P. B. (2014). A generalized, likelihood-free method for posterior estimation. Psychonomic Bulletin \& Review, 21(2), 227-250.

Turner, B. M., Sederberg, P. B., Brown, S. D., \& Steyvers, M. (2013). A method for efficiently sampling from distributions with correlated dimensions. Psychological Methods, 18, 368.

Vogel, E. K., Woodman, G. F., \& Luck, S. J. (2006). The time course of consolidation in visual working memory. Journal of Experimental Psychology: Human Perception and Performance, 32 (6), 1436-1451.

Wenger, M. J., \& Townsend, J. T. (2000). Basic response time tools for studying general processing capacity in attention, perception, and cognition. Journal of General Psychology, 127(1), 67-99.

Wilken, P., \& Ma, W. J. (2004). A detection theory account of change detection. Journal of Vision, $4,1120-1135$.

Woodman, G. F., \& Luck, S. J. (1999). Electrophysiological measurement of rapid shifts of attention during visual search. Nature, 400, 867-869.

Yang, C.-T. (2011). Relative saliency in change signals affects perceptual comparison and decision processes. Journal of Experimental Psychology: Human Perception and Performance, 37(6), 1708-1728.

Yang, C.-T., Altieri, N., \& Little, D. R. (2018). An examination of parallel versus coactive processing accounts of redundant target audiovisual signal processing. Journal of Mathematical Psychology, 82, 138-158.

Yang, C.-T., Chang, T.-Y., \& Wu, C.-J. (2013). Relative change probability affects the decision process of detecting multiple feature changes. Journal of Experimental Psychology: Human Perception and Performance, 39(5), 1365-1385.

Yang, C.-T., Hsu, H.-Y., \& Yeh, Y.-Y. (2011). Relative salience affects the process of detecting changes in orientation and luminance. Acta Psychologica, 138, 377-389. 
1728 Yang, C.-T., Little, D. R., \& Hsu, C.-C. (2014). The influence of cuing on attentional focus in 1729 perceptual decision making. Attention, Perception \&3 Psychophysics, 76(8), 2256-2275.

1730 Yang, H., Little, D. R., Eidels, A., \& Townsend, J. T. (2019). Survivor interaction contrasts for 1731 error response times: Non-parametric contrasts for serial and parallel systems. Manuscript in 1732 preparation. Indiana University..

1733 Zhang, W., \& Luck, S. J. (2008). Discrete fixed-resolution representations in visual working memory. 1734 Nature, 453(7192), 233-235. 


\section{Appendix A}

Tests of Stochastic Dominance

Table A1 shows KS-tests for Experiment 1. Stochastic dominance is as expected if $p<.05$ in the first four columns and $p>.05$ in the last four columns. Violations of stochastic dominance are bolded.

Table A1

Significance of KS-tests (p-values) of stochastic dominance in Experiment 1

\begin{tabular}{ccccccccc}
\hline & $\mathrm{HH}>\mathrm{HL}$ & $\mathrm{HH}>\mathrm{LH}$ & $\mathrm{HL}>\mathrm{LL}$ & $\mathrm{LH}>\mathrm{LL}$ & $\mathrm{HH}<\mathrm{HL}$ & $\mathrm{HH}<\mathrm{LH}$ & $\mathrm{HL}<\mathrm{LL}$ & $\mathrm{LH}<\mathrm{LL}$ \\
\hline $\mathrm{O} 1$ & .02 & .01 & $<.001$ & $<.001$ & .97 & .99 & .99 & .99 \\
$\mathrm{O} 2$ & $\mathbf{. 3 1}$ & $<.001$ & $<.001$ & $<.001$ & .69 & 1.00 & 1.00 & 0.99 \\
$\mathrm{O} 3$ & .01 & .03 & .003 & $<.001$ & 1.00 & 1.00 & .93 & .97 \\
$\mathrm{O} 4$ & $<.001$ & 0.04 & $<.001$ & $<.001$ & .93 & .93 & 1.00 & 1.00 \\
$\mathrm{O} 5$ & $<.001$ & $\mathbf{. 5 3}$ &. $\mathbf{1 1}$ & $<.001$ & 1.00 & .34 & .94 & 1.00 \\
\hline $\mathrm{A} 1$ & .04 &. $\mathbf{8 7}$ & .04 & $<.001$ & .72 & .55 & .98 & .96 \\
$\mathrm{~A} 2$ & $<.001$ & $<.001$ & $<.001$ & $<.001$ & 1.00 & 1.00 & 0.94 & .996 \\
$\mathrm{~A} 3$ & $<.001$ & $<.001$ & $<.001$ & $<.001$ & .996 & 1.00 & .983 & .983 \\
$\mathrm{~A} 4$ & $<.001$ & $<.001$ & .001 & $<.001$ & 1.00 & .963 & .983 & .936 \\
$\mathrm{~A} 5$ & $<.001$ & $<.001$ & $<.001$ & .001 & 1.00 & 1.00 & 1.00 & .999 \\
\hline
\end{tabular}

$\mathrm{O}=\mathrm{OR}$ and $\mathrm{A}=\mathrm{AND}$. 
Appendix B

Survivor Functions

1738 Figures B1 and B2 show survivor functions for the OR and AND task, respectively, for 1739 Experiment 1. 

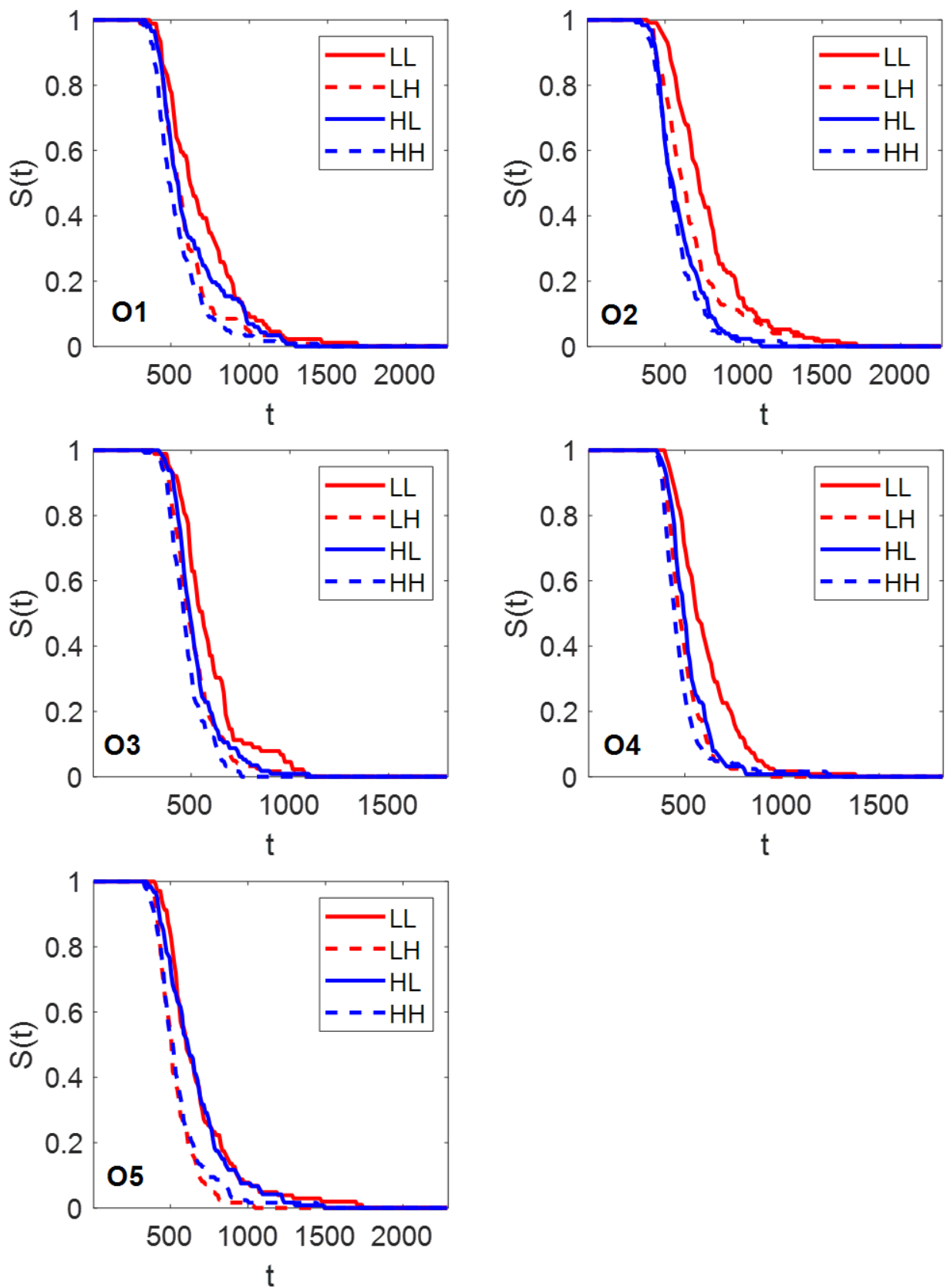

Figure B1. Survivor functions for individual participants in Experiment 1: OR Task 

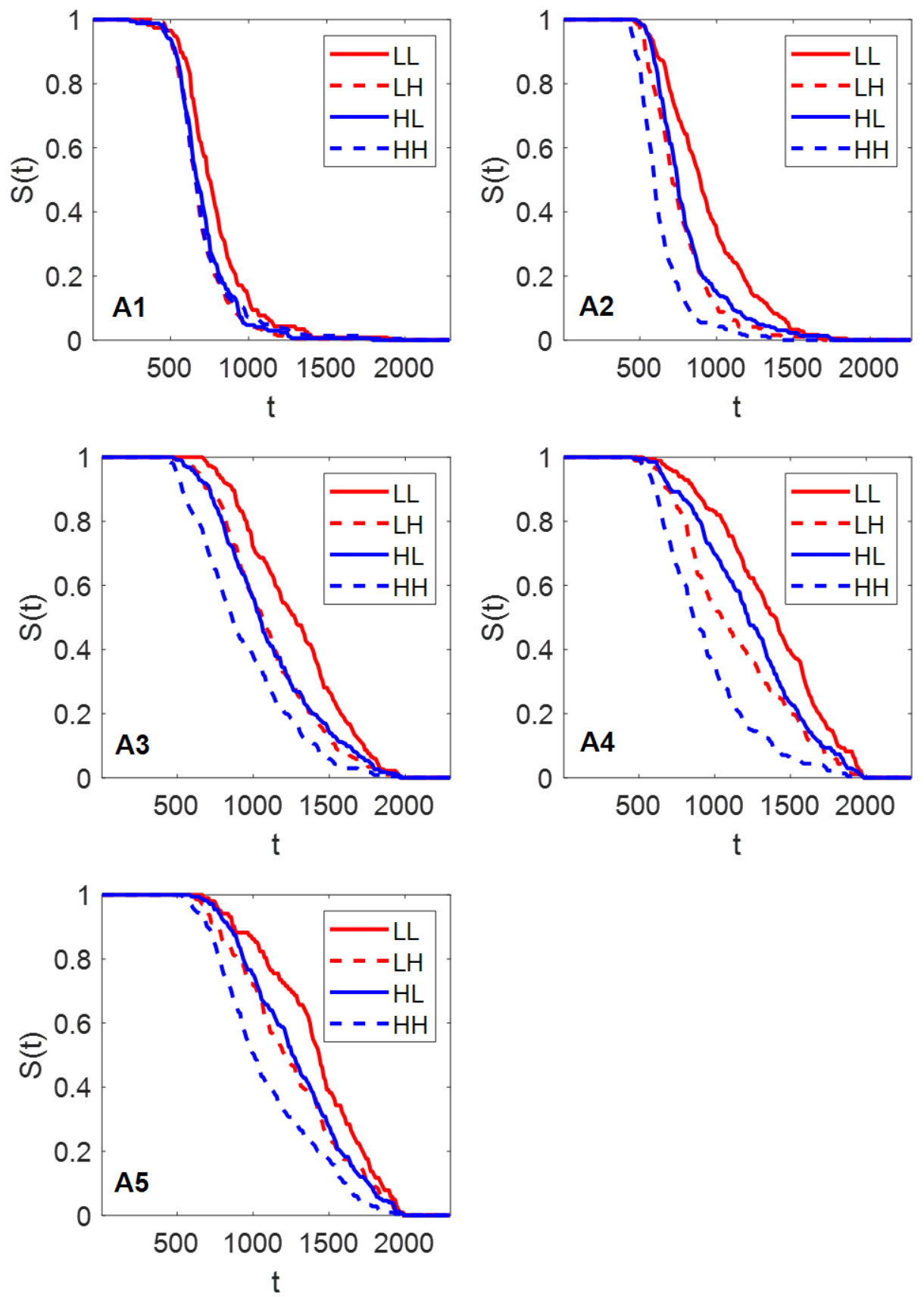

Figure B2. Survivor functions for individual participants in Experiment 1: AND Task 


\title{
Supplementary Material: Characterizing categorization decision making using visual short term memory representations
}

\author{
Anthea G. Blunden, Dylan Hammond, Piers D. L. Howe, and Daniel \\ R. Little \\ The University of Melbourne
}

\begin{abstract}
This document contains the supplementary material for Blunden, A. G., Hammond, D., Howe, P. D. L. \& Little, D. R. (2020). Characterizing categorization decision making using visual short term memory representations. Manuscript submitted for publication.
\end{abstract}

\section{AND Task: Single change trials}

The single change trials in the AND task offer additional non-parametric diagnostic information (Fifić, Little, \& Nosofsky, 2010; see also Little, Eidels, Fific, \& Wang, 2015, 2018). Diagnostic predictions for each architecture are shown in Figure 1.

In a fixed-order serial self-terminating model, one dimension (either left disc or right disc) is always processed first, followed, if necessary, by the other dimension. If the first dimension that is processed does not involve a change, then a decision can be made and the process will self-terminate. If, however, the first dimension that is processed involves a change, then processing must continue to the second dimension. This leads to the general RT prediction that the first-processed dimension will be approximately the same, whereas the second-processed dimension will be comparatively longer (as it must wait until the first process is finished before it can begin). Further, RTs for the high-magnitude item for the second-processed dimension are comparatively shorter than the low-magnitude item. This is because identifying a large magnitude change is easier than identifying a low magnitude change and so the time taken to switch from the first-processed dimension to the secondprocessed dimension should be shorter for the HS/SH items.

The single-change items for the AND task also allow for distinction between fixedorder and mixed-order serial processing. While fixed-order processing assumes that the same dimension is always processed first, a mixed-order model assumes that there is variation in which dimension is processed first from trial to trial. Like the fixed-order self-terminating model, the mixed-order model predicts that the redundant stimulus (SS) will have the shortest processing time (as both dimensions lead to a self-terminating stopping rule regardless of which is processed first). The first-processed dimension is still shorter that the second-processed dimension (again, as the second-processed dimension must wait for the first-processed dimension to complete). However, in a mixed-order model, the processing 

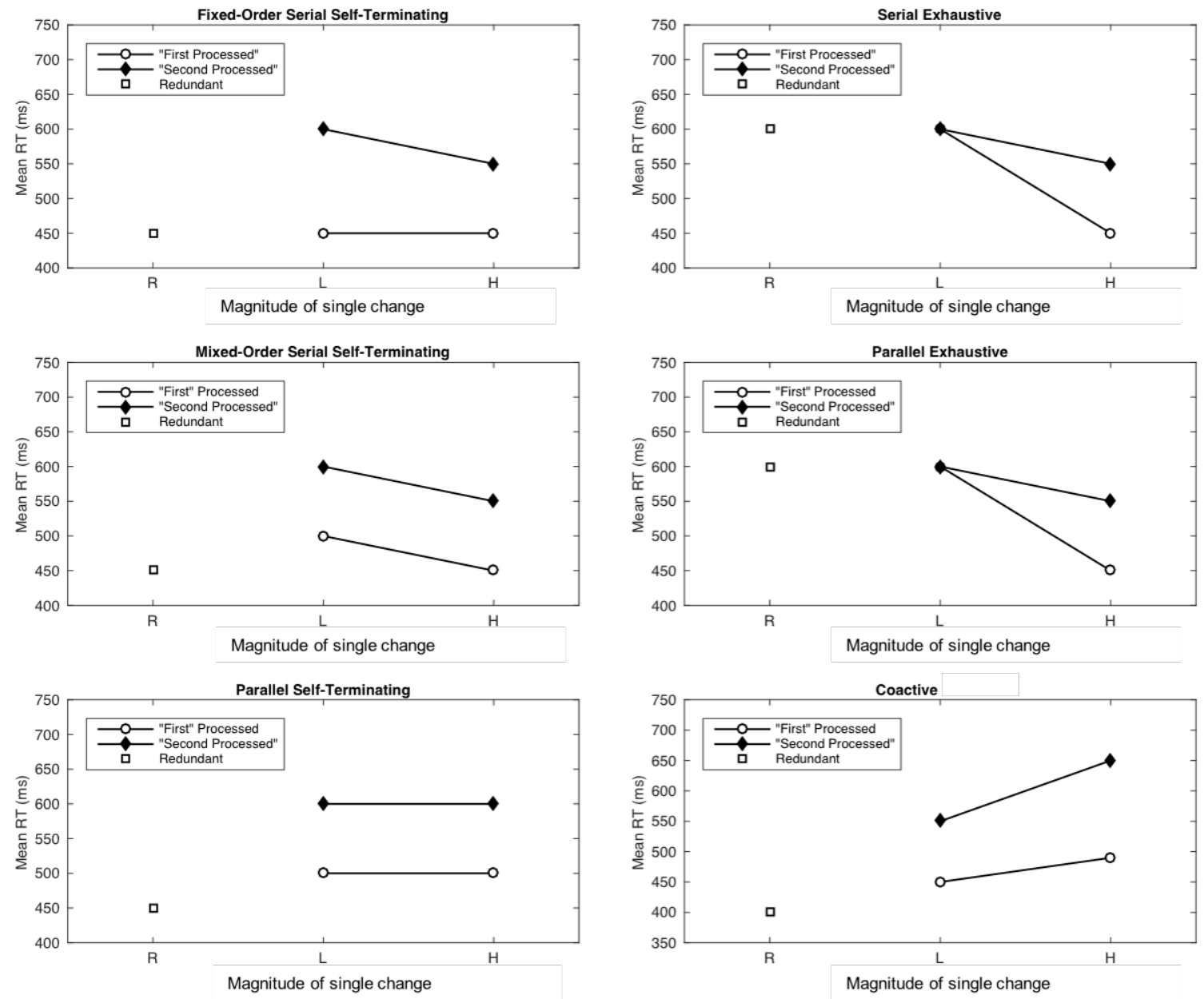

Figure 1. RT predictions for the single-change and no-change items. $\mathrm{R}=$ Redundant Stimulus, $\mathrm{L}=$ Low salience change, $\mathrm{H}=$ High salience change. 
time for the high-magnitude stimulus is shorter for both first- and second-processed dimensions. This is because, on average, the time taken to switch from one dimension to the other dimension will be shorter for high magnitude changes compared to low magnitude changes.

For the parallel self-terminating model, processing time is equivalent to the minimum processing time to detect "no-change" across both dimensions. That is, the time it takes to determine whether SL or SH contains an item which has not changed is equivalent for both stimuli. This is also the case with LS or HS. The RTs for low-magnitude and high-magnitude items are therefore equivalent, however, the redundant stimulus should have a faster processing time due to statistical facilitation (Raab, 1962; as there are more chances to self-terminate). Note that parallel and coactive models, the distinction between first-processed and second-processed is less relevant; however, it may be the case that one location is processed faster than the other. This is not a strong or central prediction of the models.

For the serial and parallel exhaustive models, both dimensions must be processed regardless of whether one, or both, dimensions do not involve a change. For the serial exhaustive model, this means that the total processing time comprises the RTs for each individual dimension. The RT for the redundant stimulus should therefore be longer than for the self-terminating models. The parallel exhaustive model predicts that the processing time is equivalent to the maximum processing time across both dimensions. Again, redundant and interior item RTs are longer than for the self-terminating models.

Finally, a general prediction of the coactive model is that the low-magnitude item RTs will be shorter than the high-magnitude items. In the coactive model, each stimulus is represented by a bivariate normal distribution. As one moves from the high-magnitude items towards the left-most corner of the decision space, a greater proportion of the distribution will lie in the single/no-change region. Hence, the chance that a sample will come from the no-change distribution increases for the redundant and low-magnitude items compared to the high-magnitude items, also resulting in a shorter RT for the redundant stimulus and low-magnitude items.

For the AND Task, the mean RTs for the single change items are displayed in Figure 2. For most participants, the RTs for the redundant stimulus was shorter than the other stimuli, suggesting a self-terminating stopping rule. This difference was predominantly significant, excepting the the HS-R comparison for A2 and the SL-R comparison for A4. For A5 the RT for the redundant stimulus was longer than the HS and LS items. For A2 and A3 the RTs for the low-magnitude items were longer than the high-magnitude items, which is consistent with serial processing. However, for A4 the RTs for the low-magnitude items were shorter than the high-magnitude items which could suggest coactive processing. For A5 RTs for the low-magnitude item were shorter on one dimension and longer on the other.

For AND Task single change items, we conducted a series of planned $t$ tests comparing low-magnitude and high-magnitude items on both dimensions and comparing the redundant stimulus to the other single change items (see Table 1). For most items the RTs for the redundant stimulus was significantly shorter than the other stimuli, indicating a self-terminating stopping rule ${ }^{1}$.

\footnotetext{
${ }^{1}$ The HS item for A2, SL item for A4 and LS and HS item for A5 were non-significant.
} 

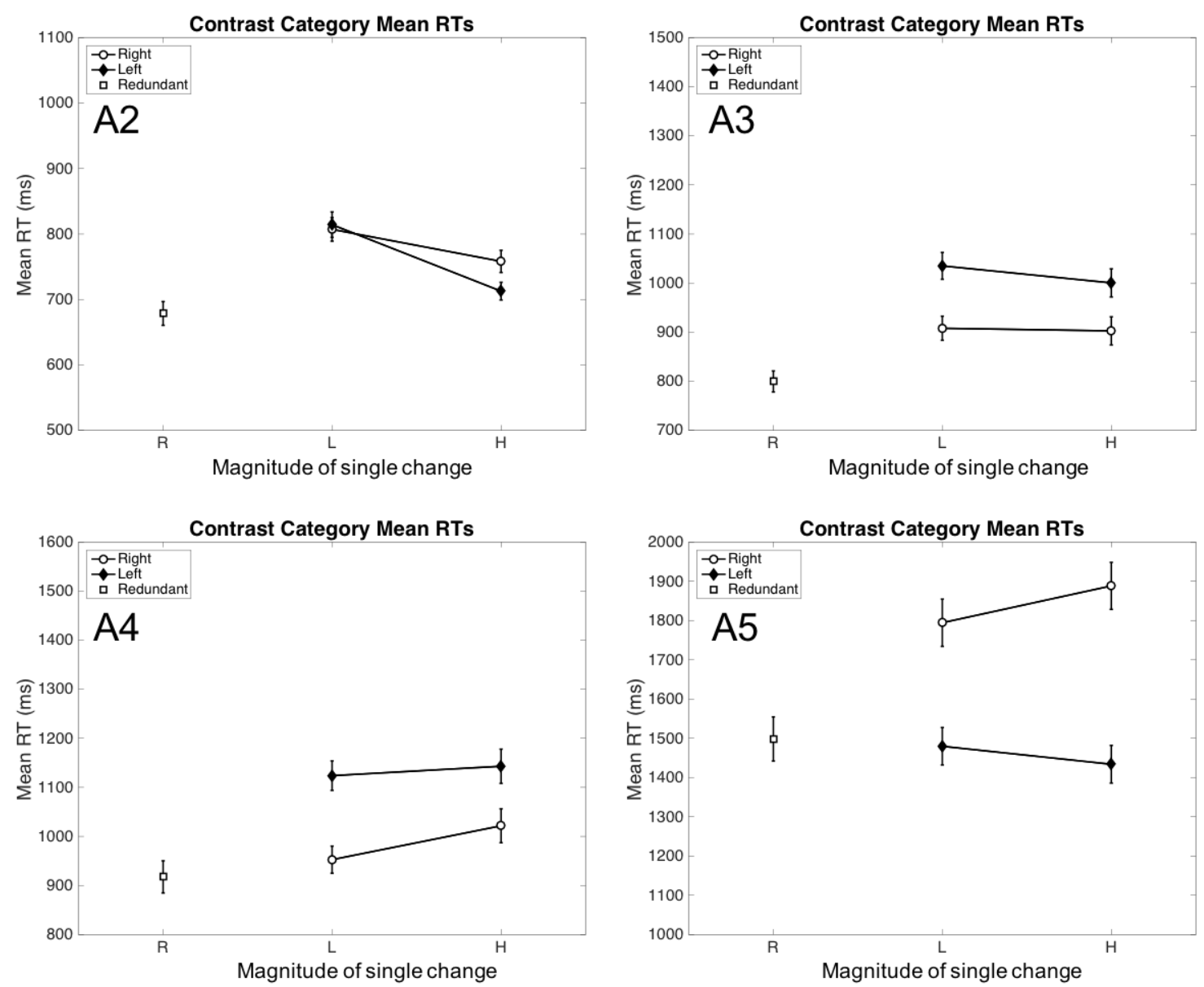

Figure 2. Observed single change item mean RTs for individual participants in Experiment 1: AND Task. Error bars represent one standard error. $\mathrm{R}=$ redundant stimulus, $\mathrm{I}=$ interior stimulus, $\mathrm{E}=$ exterior stimulus.

For A2 the low-magnitude item RTs were significantly longer than the high-magnitude item RTs, indicating serial processing. For the remaining participants there were no significant differences between low-magnitude and high-magnitude items which is consistent with parallel processing, however, caution must be exercised when extrapolating from a null result. 
Table 1

Single Change Item Statistical Results for Individual Participants in Experiment 1: AND task

\begin{tabular}{|c|c|c|c|c|c|c|c|c|}
\hline Stimulus Pair & Mdiff & $t$ & $d f$ & $p$ & Mdiff & $t$ & $d f$ & $p$ \\
\hline & \multicolumn{4}{|c|}{$\mathrm{A} 2$} & \multicolumn{4}{|c|}{ A3 } \\
\hline HS - LS & -101.58 & -4.34 & 331 & $<.001$ & -34.68 & -0.88 & 332 & 0.382 \\
\hline $\mathrm{SH}$ - SL & -48.97 & -1.98 & 302 & 0.049 & -5.34 & -0.14 & 310 & 0.887 \\
\hline $\mathrm{HS}-\mathrm{R}$ & 34.06 & 1.54 & 300 & 0.125 & 200.79 & 5.37 & 292 & $<.001$ \\
\hline LS - R & 135.64 & 5.01 & 287 & $<.001$ & 235.44 & 6.45 & 298 & $<.001$ \\
\hline $\mathrm{SH}-\mathrm{R}$ & 79.54 & 3.20 & 280 & 0.002 & 103.03 & 2.8 & 282 & 0.006 \\
\hline $\mathrm{SL}-\mathrm{R}$ & 128.51 & 4.99 & 278 & $<.001$ & 108.37 & 3.25 & 286 & 0.001 \\
\hline \multirow[t]{2}{*}{ Stimulus Pair } & Mdiff & $t$ & $d f$ & $p$ & Mdiff & $t$ & $d f$ & $p$ \\
\hline & \multicolumn{4}{|c|}{$\mathrm{A} 4$} & \multicolumn{4}{|c|}{$\mathrm{A} 5$} \\
\hline HS - LS & 19.19 & 0.42 & 325 & 0.676 & -45.84 & -0.68 & 279 & 0.499 \\
\hline $\mathrm{SH}-\mathrm{SL}$ & 69.09 & 1.56 & 328 & 0.120 & 93.99 & 1.11 & 201 & 0.270 \\
\hline $\mathrm{HS}-\mathrm{R}$ & 225.63 & 4.64 & 288 & $<.001$ & -64.41 & -0.88 & 258 & 0.382 \\
\hline LS - R & 206.44 & 4.62 & 295 & $<.001$ & -18.57 & -0.25 & 253 & 0.800 \\
\hline $\mathrm{SH}-\mathrm{R}$ & 104.23 & 2.14 & 294 & 0.033 & 390.17 & 4.75 & 220 & $<.001$ \\
\hline $\mathrm{SL}-\mathrm{R}$ & 35.15 & 0.83 & 292 & 0.410 & 296.18 & 3.59 & 213 & $<.001$ \\
\hline
\end{tabular}

\section{Comparison of the estimated perceptual variance in the free-variance model}

To examine whether perceptual variability differed across different change magnitudes (no change, low change, or high change), we extracted the perceptual variability parameters from the best fitting models for most participants from each task. These were the parallel self-terminating model in OR task and the serial self-terminating model in the AND task. We then subtracted the no change variability estimates from the low change variability estimates and the low change variability estimates from the high change variability estimates for the left and right locations. These posterior difference estimates are shown in Figure 3 along with the estimated 95\% HDIs. In all cases the HDI's overlap 0. 

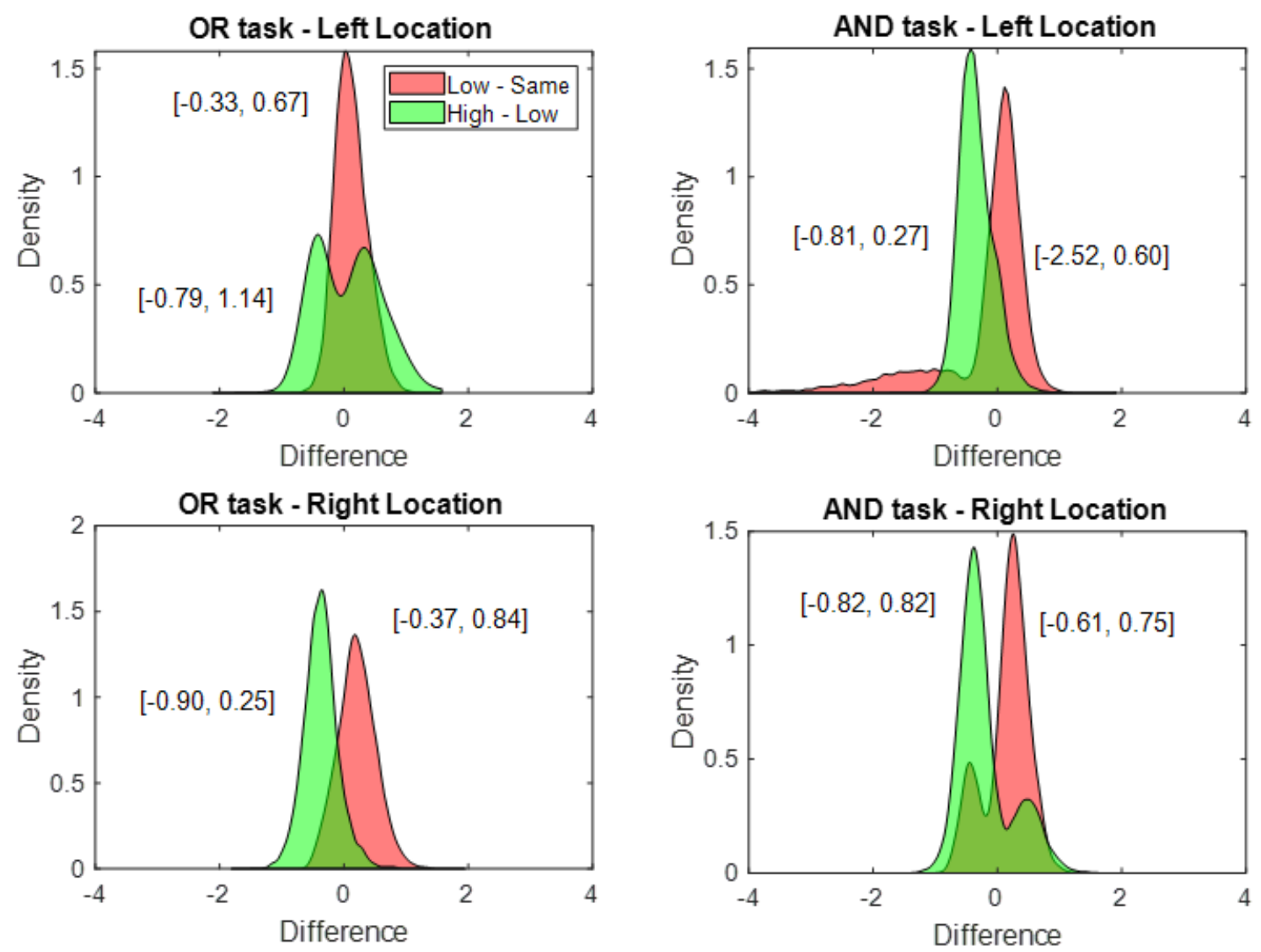

Figure 3. Posterior distributions over the differences in perceptual variance.

\section{Individual Capacity Estimates for Experiment 2}

In the primary manuscript, we reported the group capacity estimates. Here we present the individual capacity estimates for each individual participant. 

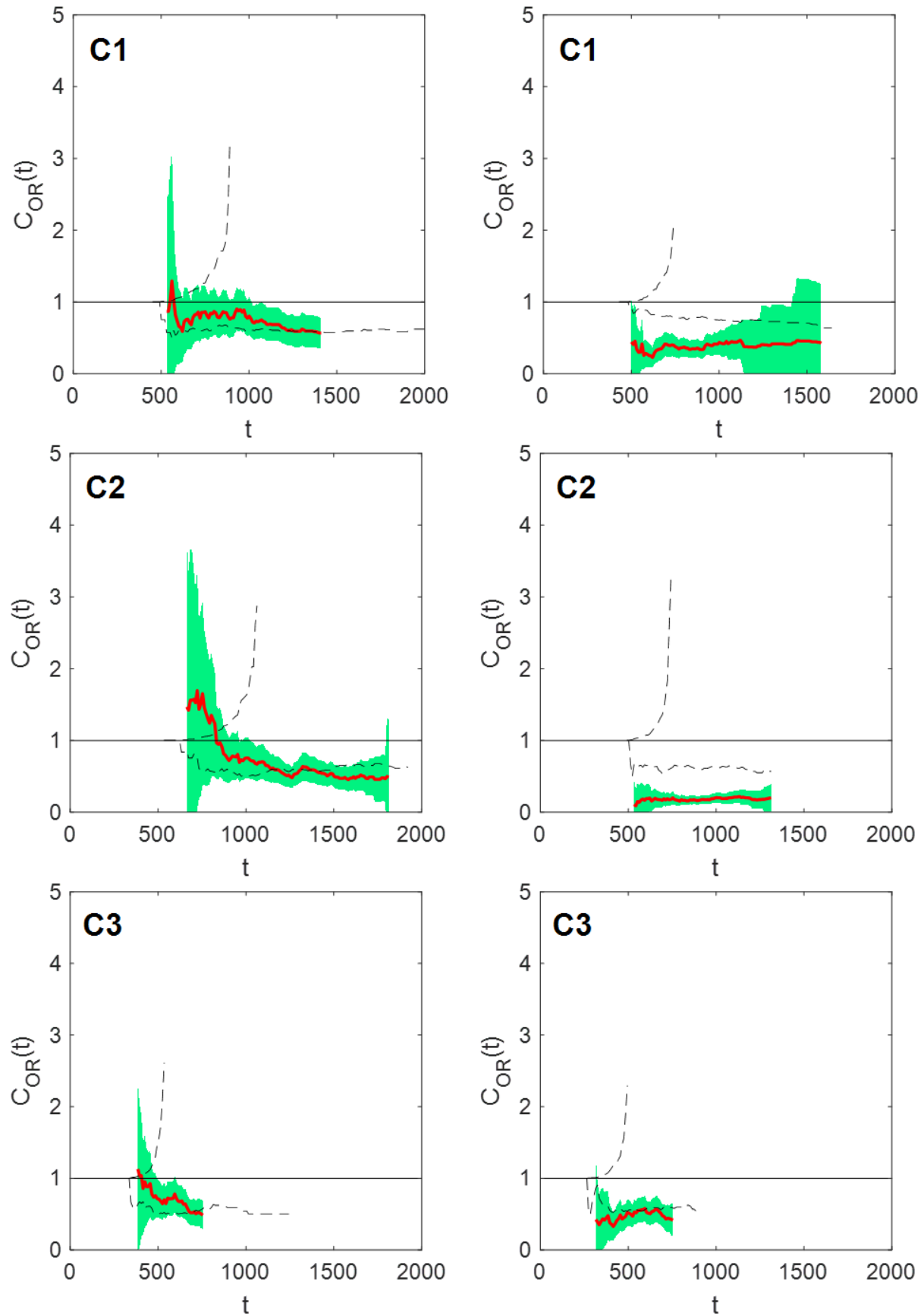

Figure 4. Capacity estimates for each individual participant in the OR condition. Red line represents the capacity coefficient. Black dotted lines represent the Miller (upper) and Grice (lower) bounds, respectively. Left column: redundant target vs single targets+same. Right column: redundant target vs single targets alone. 

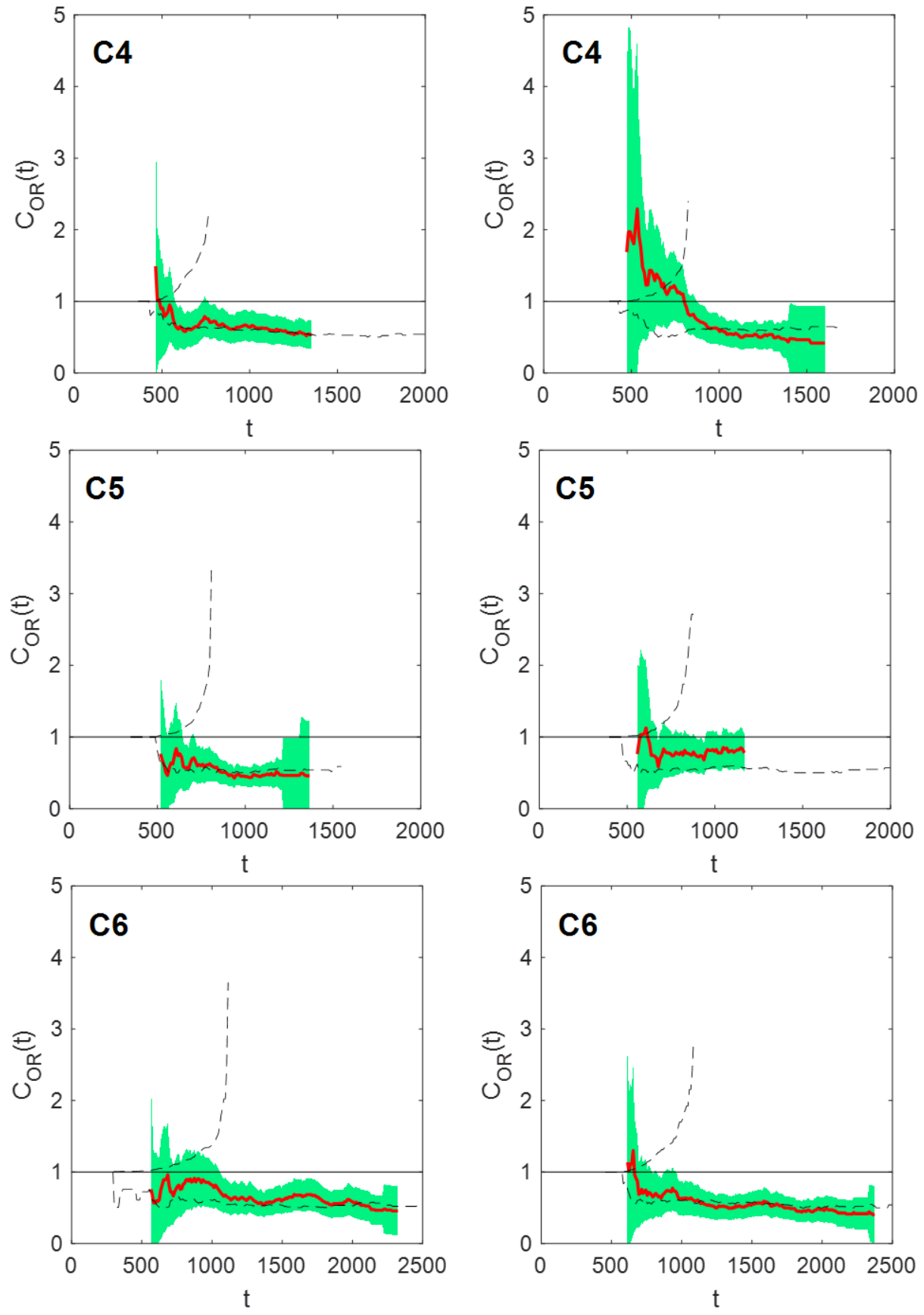

Figure 4. Continued. 

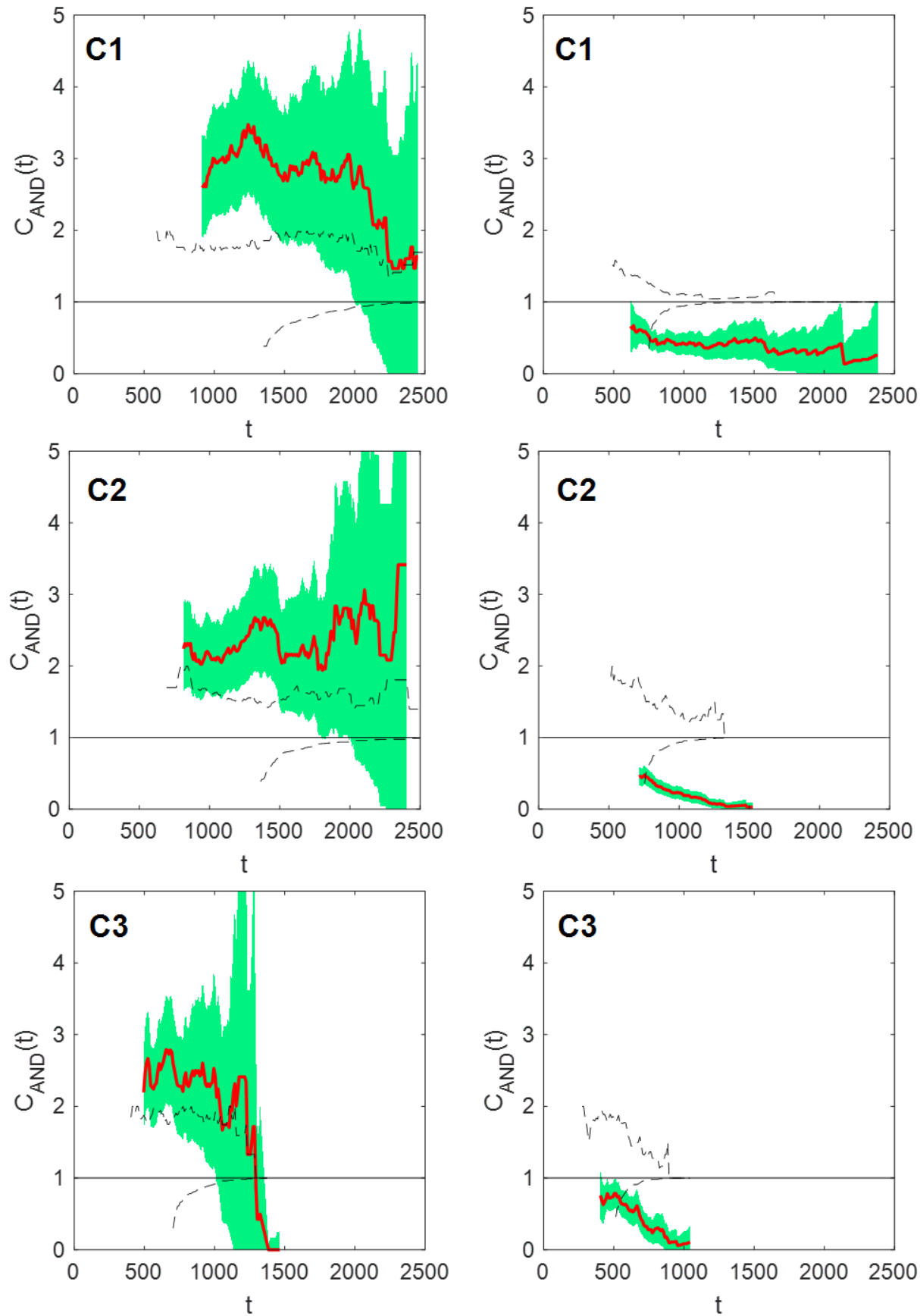

Figure 5. Capacity estimates for each individual participant in the AND condition. Red line represents the capacity coefficient. Black dotted lines represent the Miller (upper) and Grice (lower) bounds, respectively. Left column: redundant target vs single targets+same. Right column: redundant target vs single targets alone. 

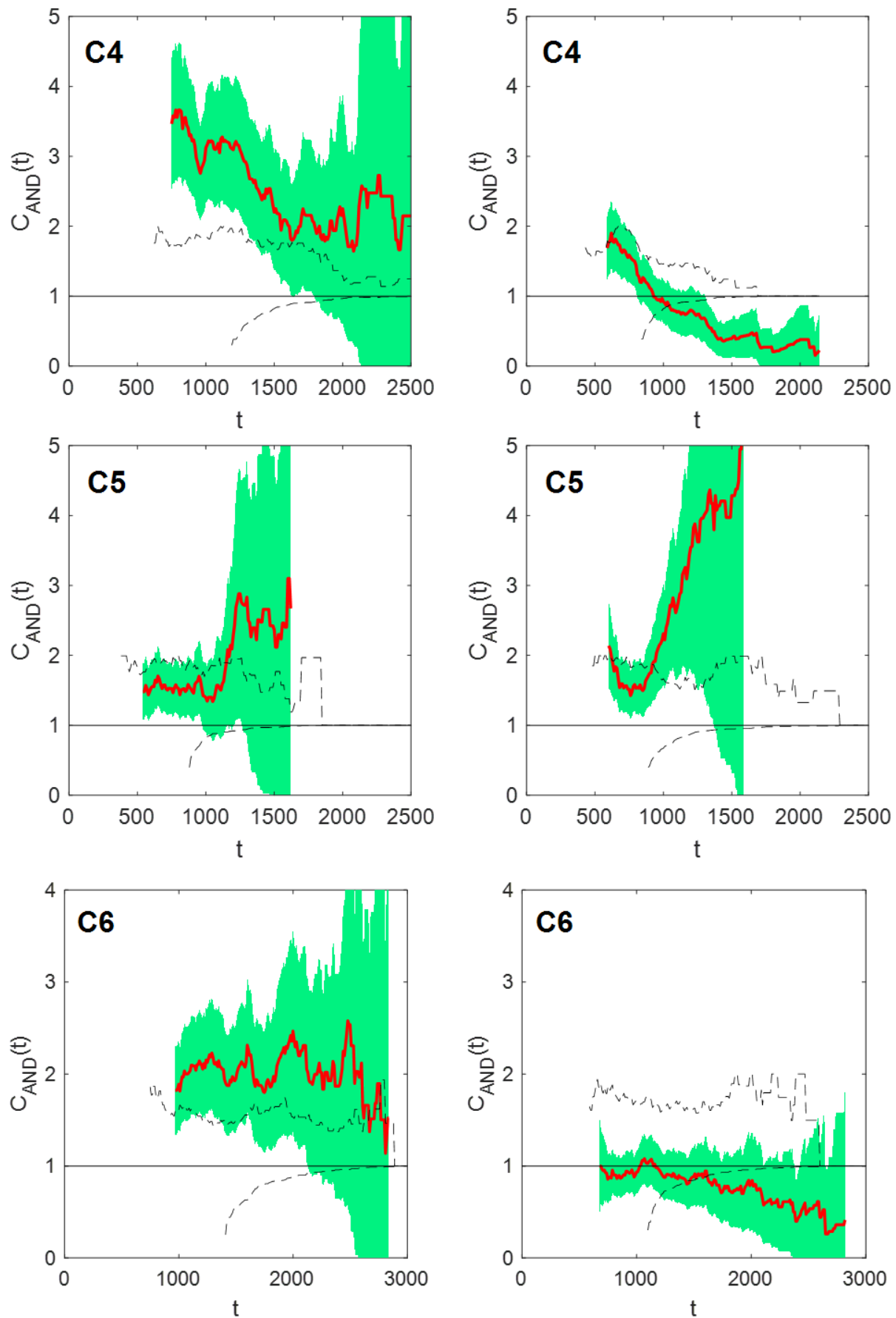

Figure 5. Continued. 


\section{Posterior Predictive Distributions}

In the following posterior predictive distribution plots, each panel captures the data for a single item. The RTs are plotted as a histogram; positive values indicate target category response, and negative values indicate contrast category response. The four items in the top right of each figure belonged to the target category; consequently, most of the RTs should be positive, and any negative RTs indicate an error response for these items. The remaining items belonged to the contrast category and should therefore have mostly negative RTs. Any positive RTs for these items are error RTs.

For each observer, we took 40 samples of parameters from the posterior and generated predictions. Each sample prediction is plotted as a red line. The solid blue line is the prediction based on the average posterior parameters. The likelihood of the average posterior samples is used in the computation of the DIC. The data were binned into $50 \mathrm{msec}$ bins. The posterior predictive densities found using a kernel density estimate with a bandwidth of $10 \mathrm{msec}$. 


\section{OR TASK}

The following plots show the posterior predictions from the best-fitting model for each observer from the OR task.

Observer 01: Coactive-min fixed variance model. For the posterior predictions of the coactive-min fixed variance model for observer O1, see Figure 6 below.
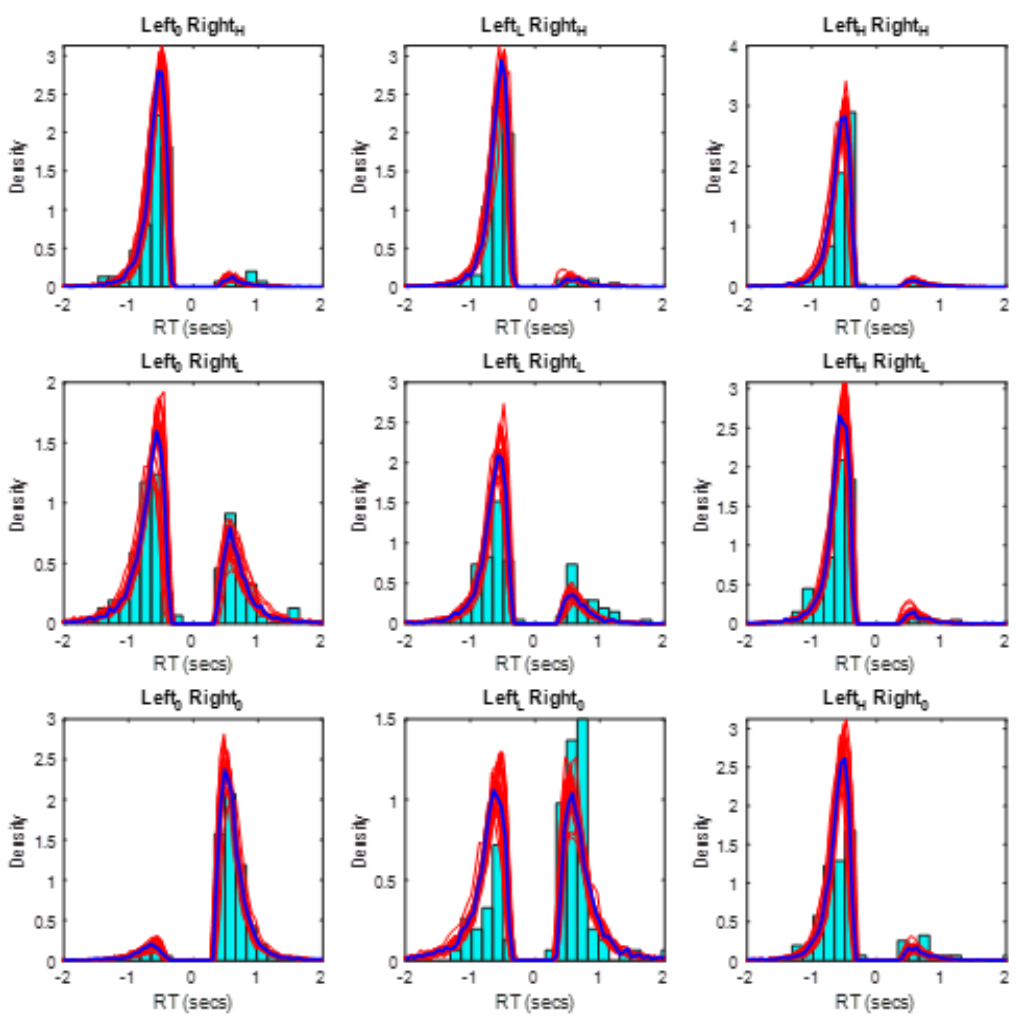

Figure 6. Posterior predictions of the coactive-min fixed variance model for observer O1. Each subplot shows the predictions for one item. Positive RTs indicate target category response. Negative RTs indicate contrast category responses. Data are plotted in the histogram. Each red line indicates a posterior sample. The solid blue line indicates the predictions using the average posterior parameter values. 
Observer O2: parallel self-terminating free variance model. For the posterior predictions from the parallel self-terminating free variance model for observer $\mathrm{O} 2$, see Figure 7 below.
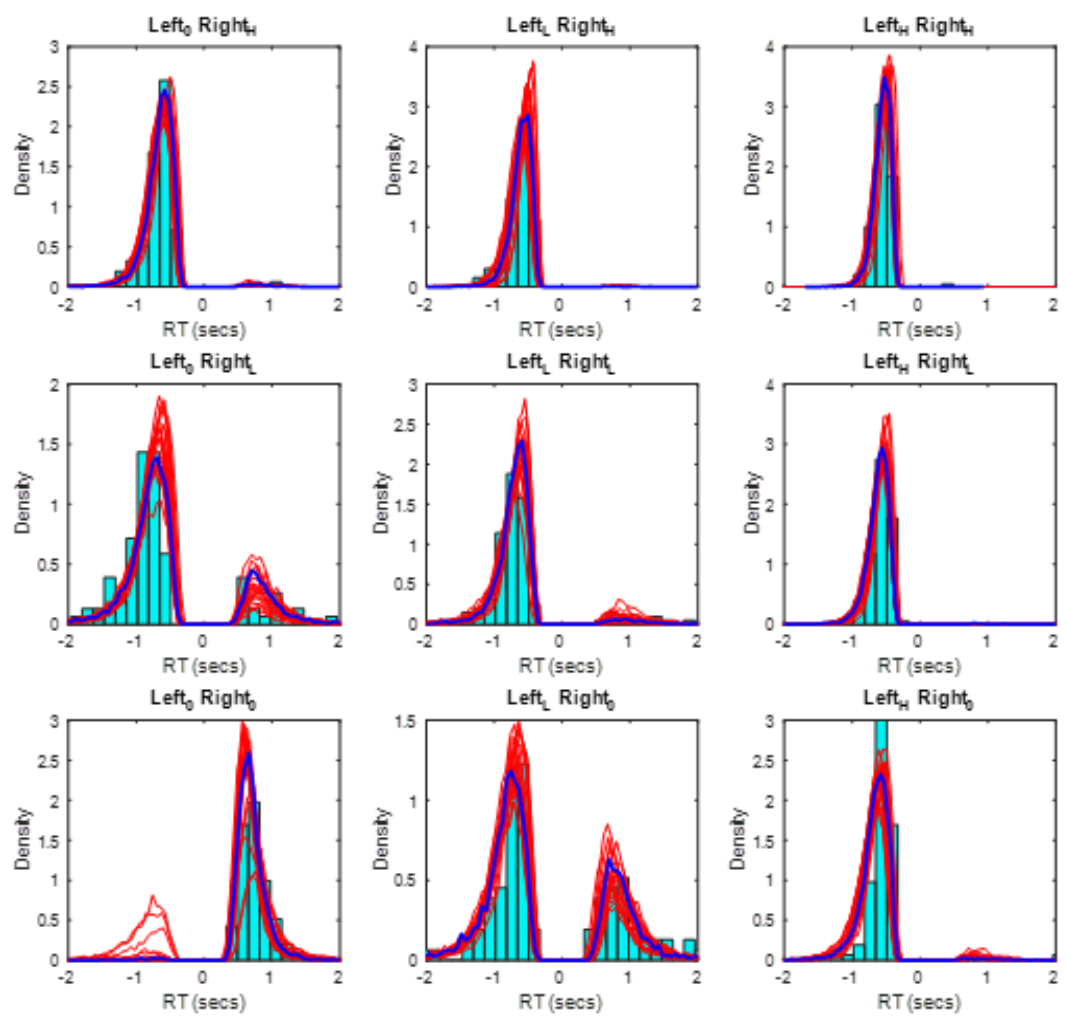

Figure 7. Posterior predictions from the parallel self-terminating free variance model for observer $\mathrm{O} 2$. 
Observer O3: parallel self-terminating fixed variance model. For the posterior predictions from the parallel self-terminating fixed variance model for observer O3, see Figure 8 below.
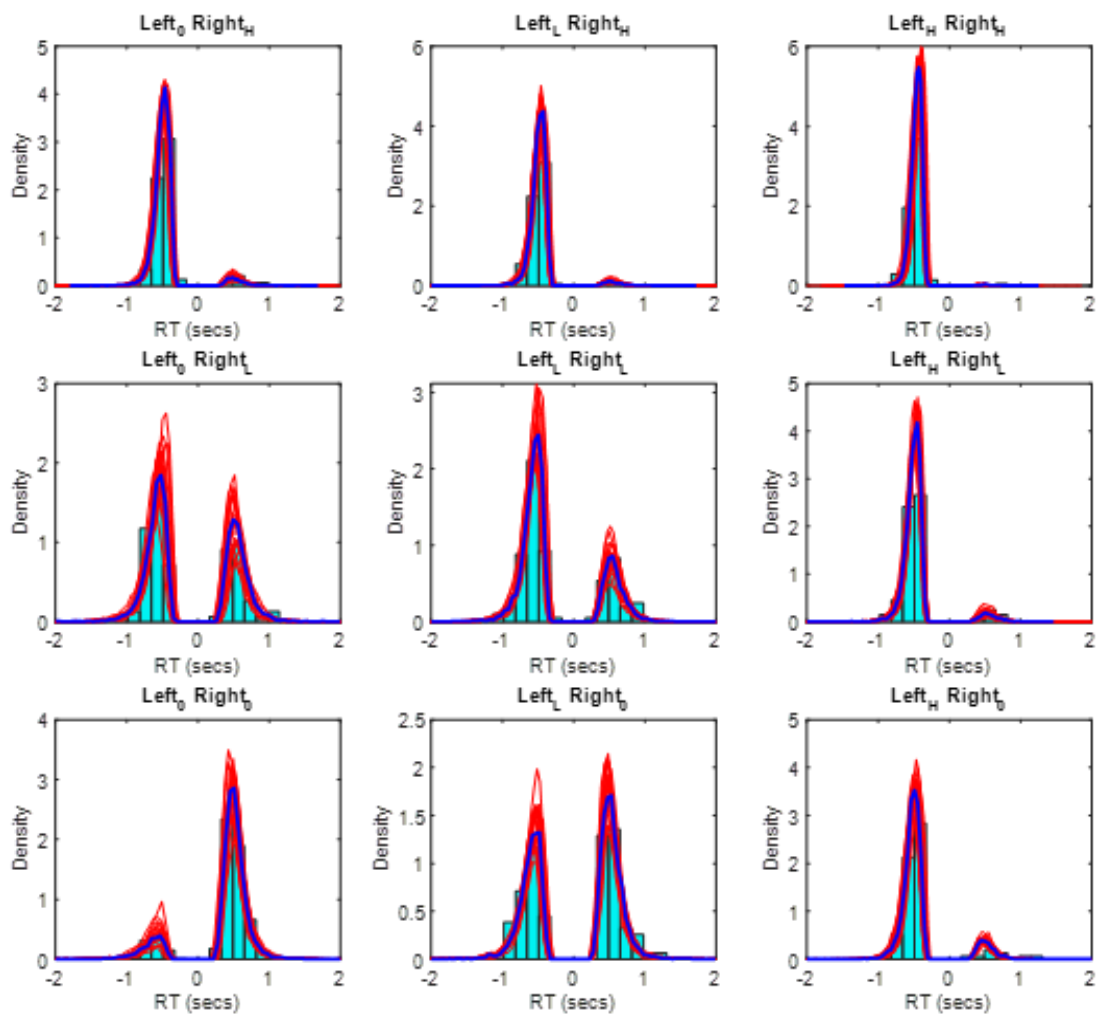

Figure 8. Posterior predictions from the parallel self-terminating fixed variance model for observer O3. 
Observer 04: parallel self-terminating fixed variance model. For the posterior predictions from the parallel self-terminating fixed variance model for observer $\mathrm{O} 4$, see Figure 9 below.
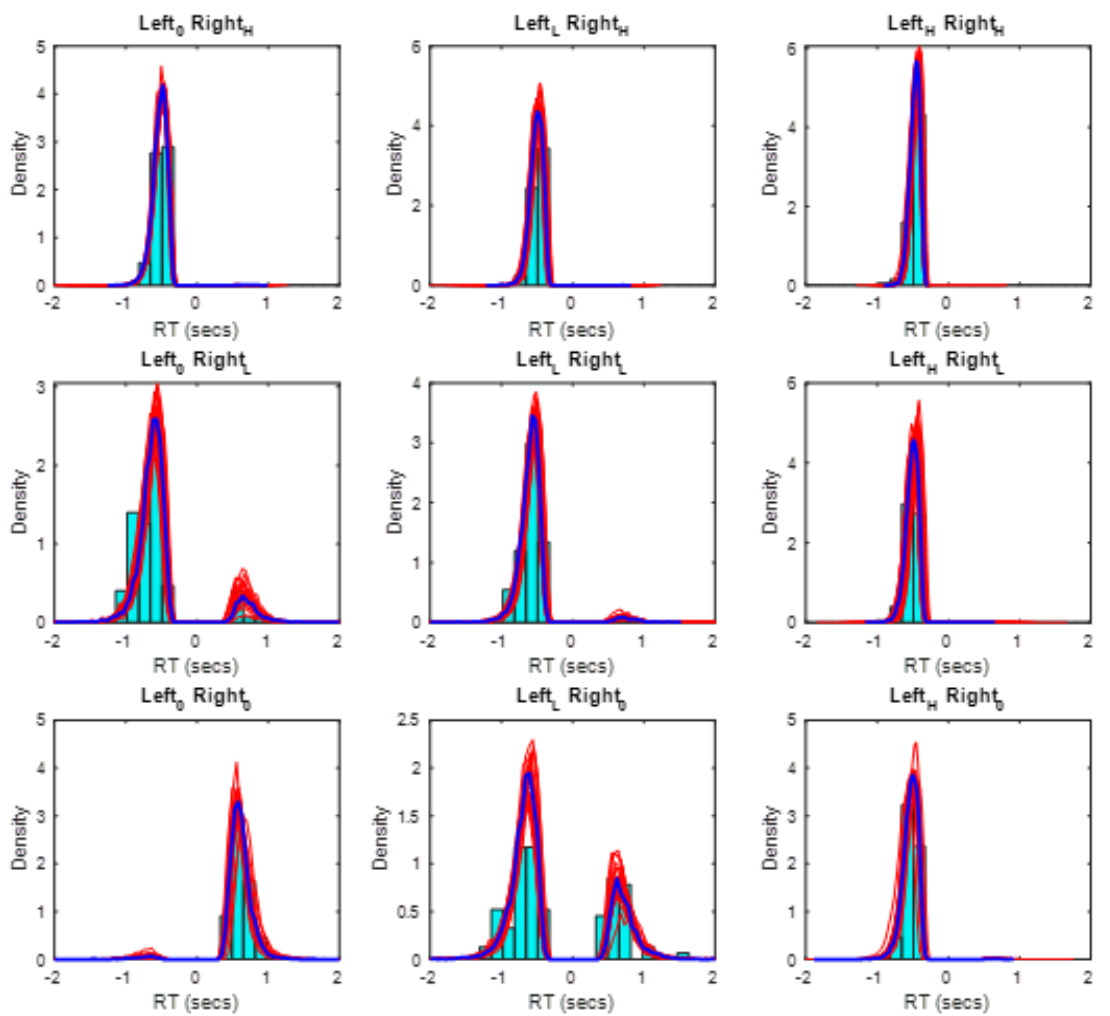

Figure 9. Posterior predictions from the parallel self-terminating fixed variance model for observer $\mathrm{O} 4$. 
Observer O5: parallel self-terminating free variance model. For the posterior predictions from the parallel self-terminating free variance model for observer O5, see Figure 10 below.
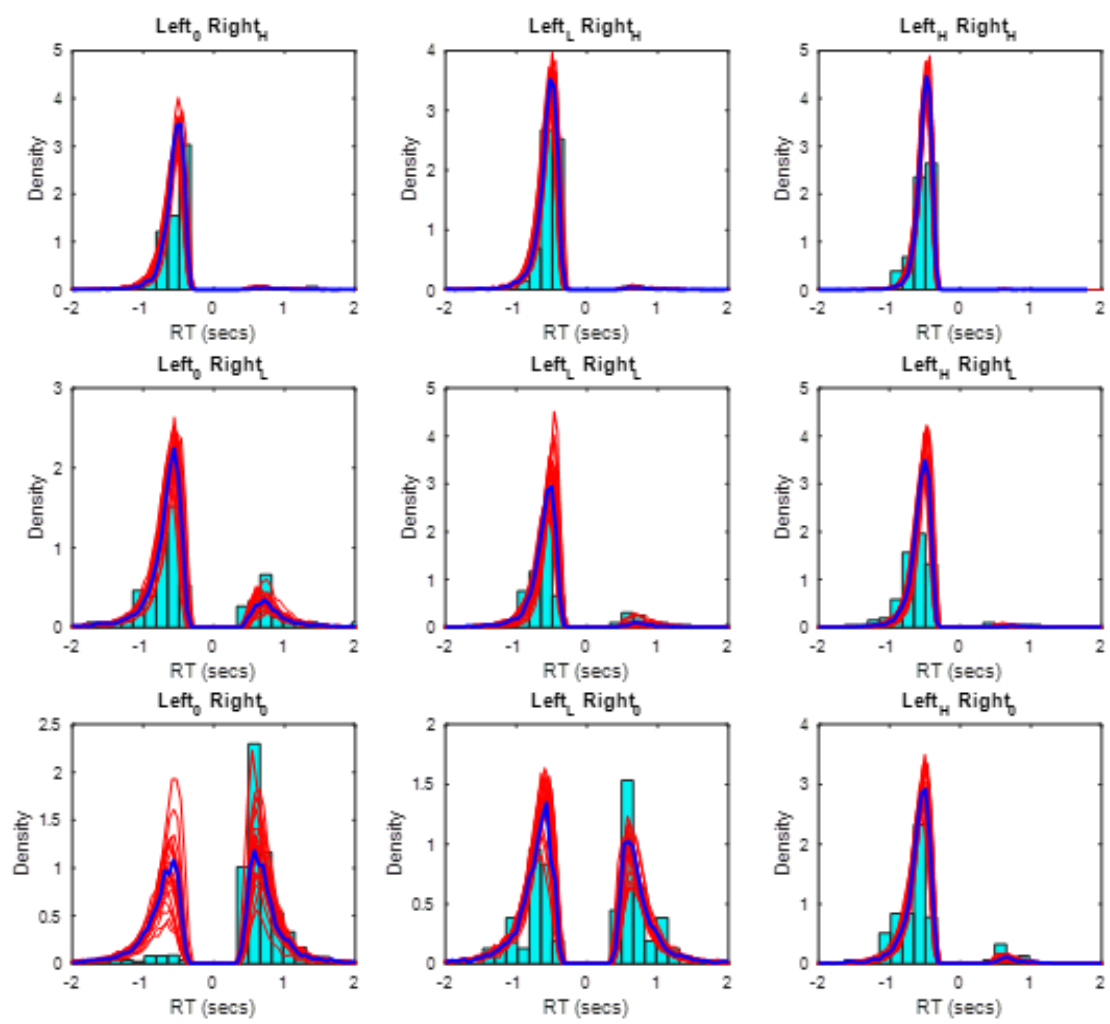

Figure 10. Posterior predictions from the parallel self-terminating free variance model for observer O5. 


\section{AND TASK}

The following plots show the posterior predictions from the best-fitting model for each observer from the AND task.

Observer A1: coactive-max fixed variance model. For the posterior predictions from the coactive-max fixed variance model for observer A1, see Figure 11 below.
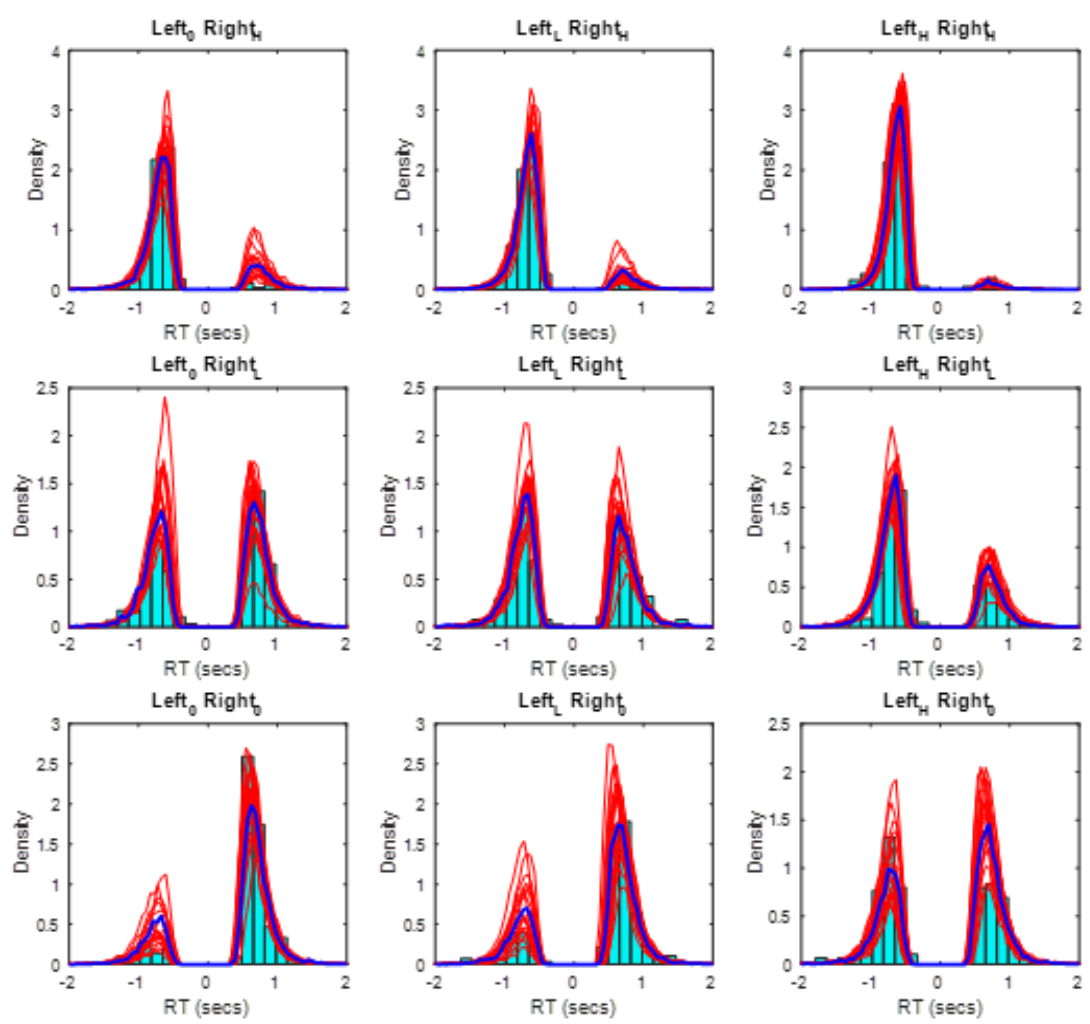

Figure 11. Posterior predictions from the coactive-max fixed variance model for observer A1. 
Observer A2: serial self-terminating free variance model. For the posterior predictions from the serial self-terminating free variance model for observer A2, see Figure 12 below.
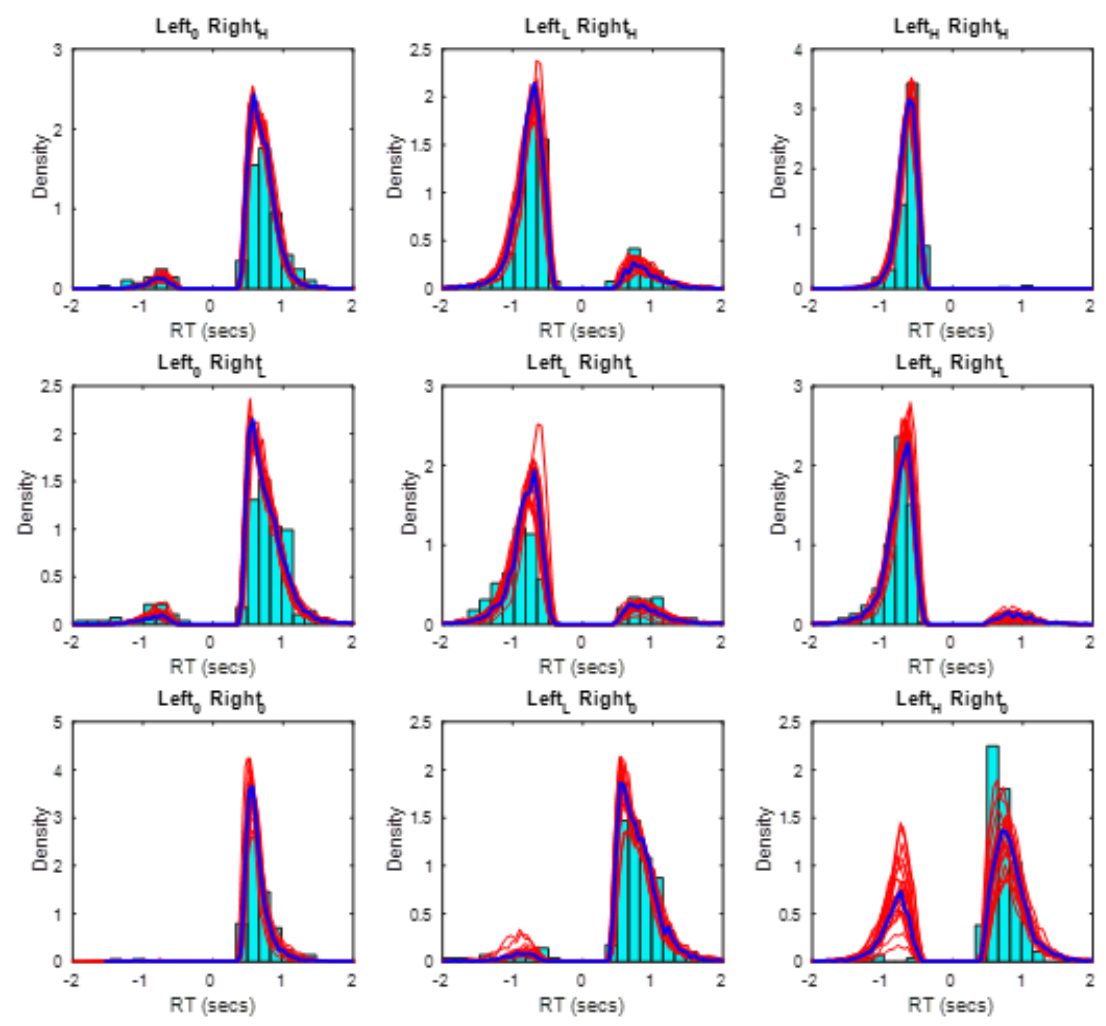

Figure 12. Posterior predictions from the serial self-terminating free variance model for observer A2. 
Observer A3: serial self-terminating fixed variance model. For the posterior predictions from the serial self-terminating fixed variance model for observer A3, see Figure 13 below.
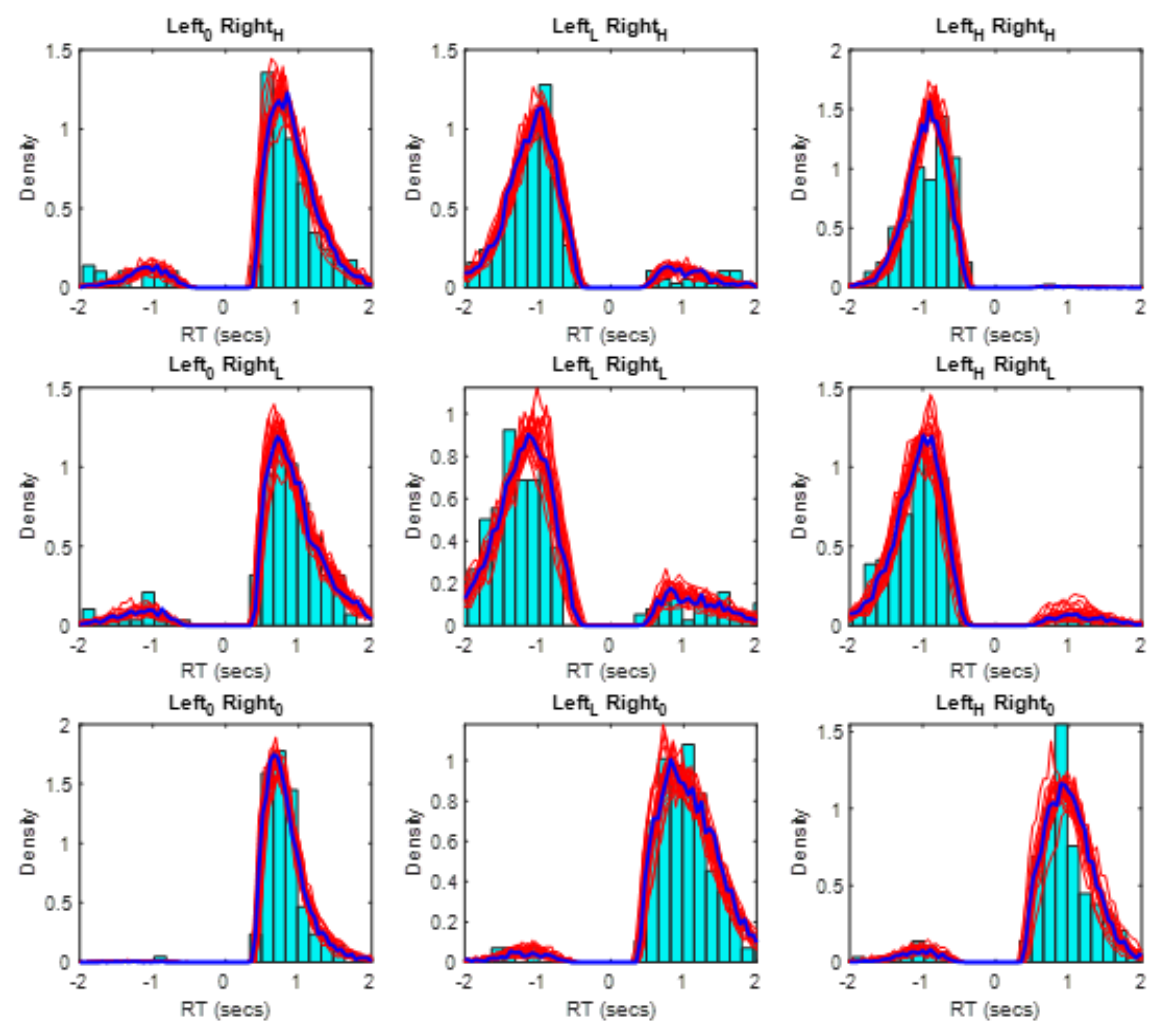

Figure 13. Posterior predictions from the serial self-terminating fixed variance model for observer A3. 
Observer A4: serial self-terminating fixed variance model. For the posterior predictions from the serial self-terminating fixed variance model for observer A4, see Figure 14 below.
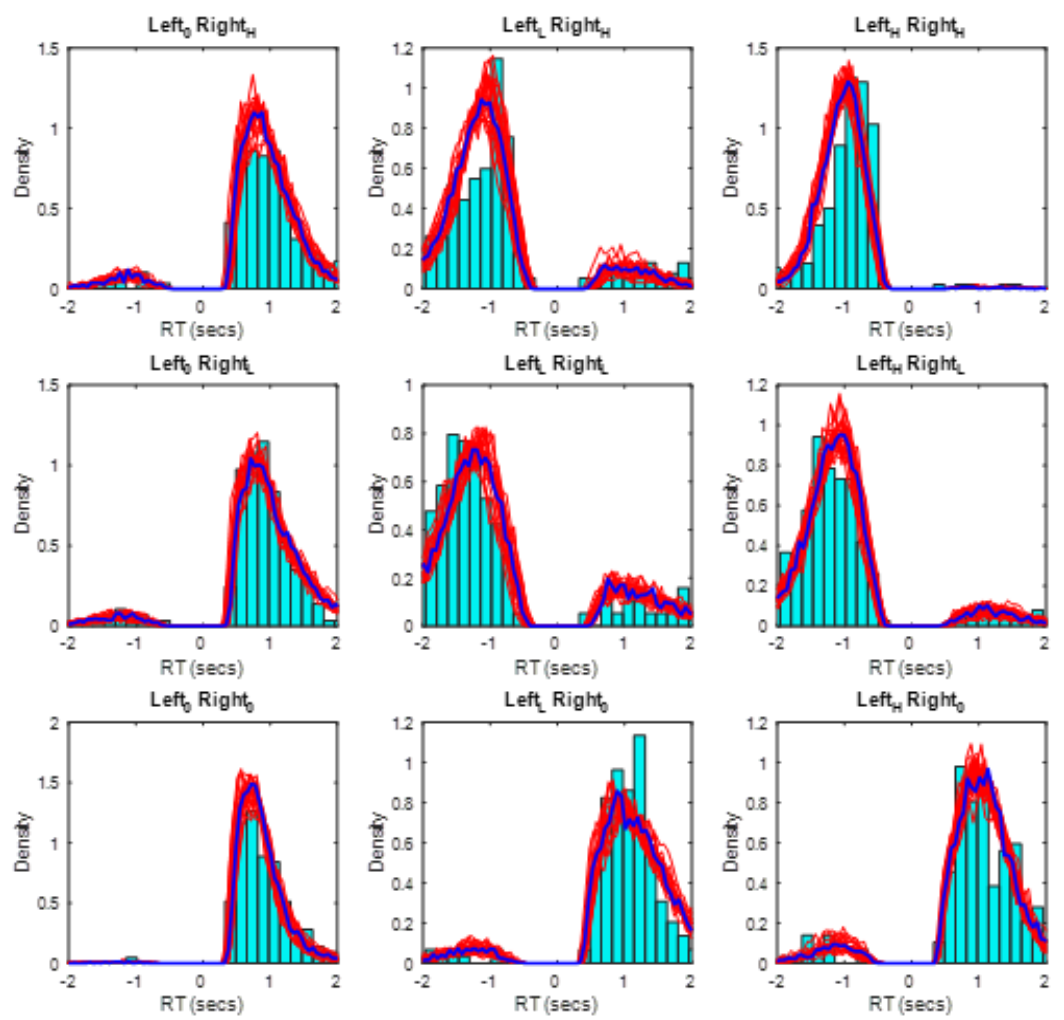

Figure 14. Posterior predictions from the serial self-terminating fixed variance model for observer A4. 
Observer A5: coactive-max fixed variance model. For the posterior predictions from the coactive-max fixed variance model for observer A5, see Figure 15 below.
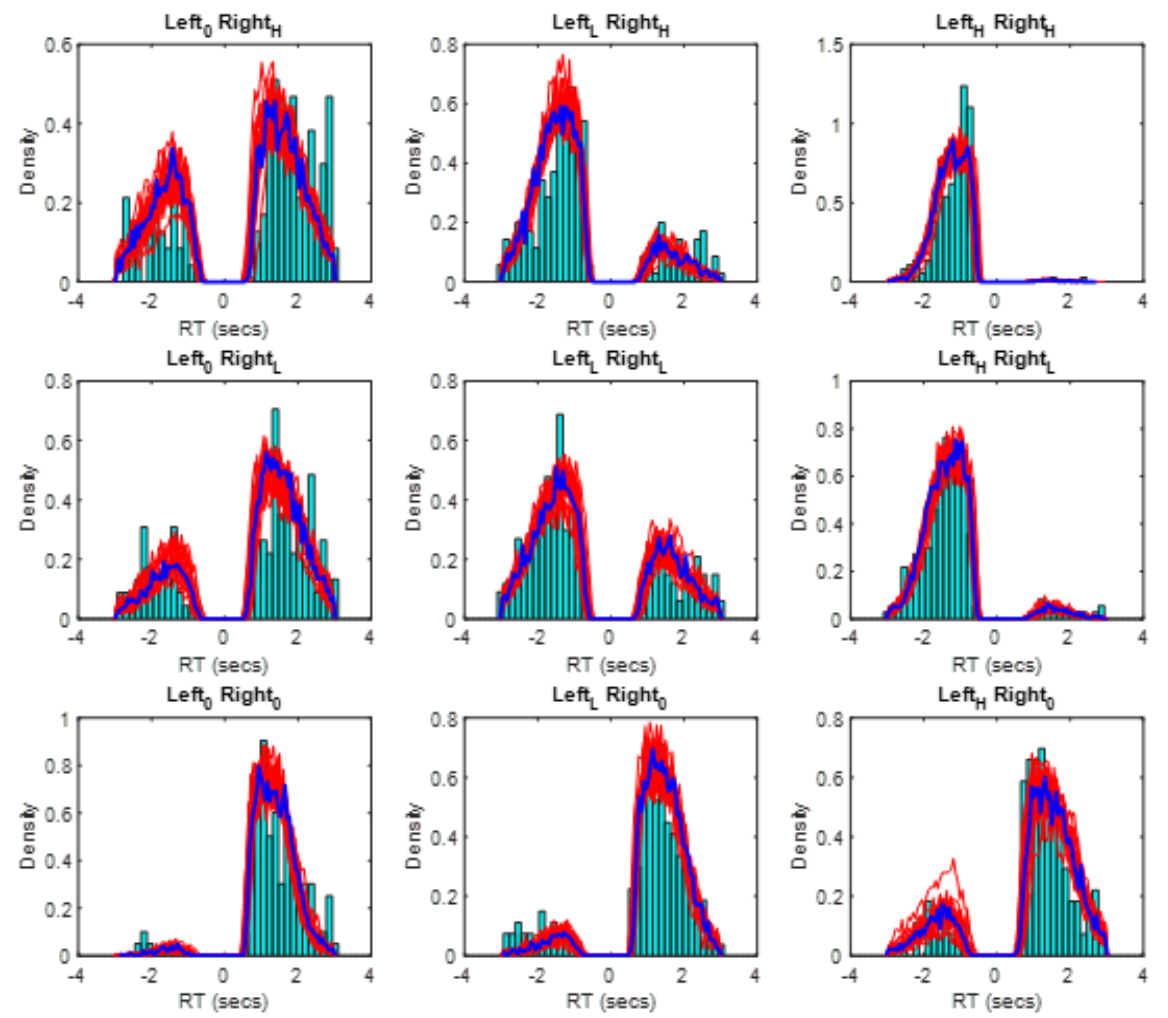

Figure 15. Posterior predictions from the coactive-max fixed variance model for observer A5. 


\section{References}

Fifić, M., Little, D. R., \& Nosofsky, R. (2010). Logical-rule models of classification response times: A synthesis of mental-architecture, random-walk, and decision-bound approaches. Psychological Review, 117, 309-348.

Little, D. R., Eidels, A., Fific, M., \& Wang, T. (2015). Understanding the influence of distractors on workload capacity. Journal of Mathematical Psychology, 68, 25-36.

Little, D. R., Eidels, A., Fific, M., \& Wang, T. (2018). How do information processing systems deal with conflicting information? differential predictions for serial, parallel and coactive processing models. Computational Brain \& Behavior, 1, 1-21.

Raab, D. (1962). Statistical facilitation of simple reaction time. Transaction of the New York Academy of Science, 43, 574-590. 